\title{
Four 'Peculiar' RRd Stars Observed by K2
}

\author{
James M. Nemec ${ }^{1,2 \star}$ and Paweł Moskalik ${ }^{3}$ \\ ${ }^{1}$ Department of Physics \&3 Astronomy, Camosun College, Victoria, British Columbia, Canada \\ ${ }^{2}$ International Statistics \& Research Corporation, Brentwood Bay, British Columbia, Canada \\ ${ }^{3}$ Nicolaus Copernicus Astronomical Center, Polish Academy of Sciences, ul. Bartycka 18, 00-716 Warszawa, Poland
}

Accepted 2021 July 2. Received 2021 July 2; in original form 2021 February 25

\begin{abstract}
Four stars pulsating simultaneously with a dominant period $P_{D} \in(0.28,0.39) \mathrm{d}$ and an additional period $P_{A} \in(0.20,0.27)$ d have been identified from among the more than 3000 RR Lyrae stars observed by the Kepler space telescope during NASA's K2 Mission. All four stars are located in the direction of the Galactic Bulge and have period ratios, $P_{A} / P_{D}$, significantly smaller than those of most double-mode RR Lyrae (RRd) stars: $P_{A} / P_{D} \in(0.694,0.710)$ vs. $P_{1} / P_{0} \in(0.726,0.748)$. Three of the stars are faint $(<V\rangle=18-20 \mathrm{mag})$ and distant and are among the 'peculiar' RRd (pRRd) stars discovered by Prudil et al. (2017); the fourth star, EPIC 216764000 (=V1125 Sgr), is a newly discovered pRRd star several magnitudes brighter than the other three stars. In this paper the high-precision long-cadence $K 2$ photometry is analyzed in detail and used to study the cycle-to-cycle light variations. The pulsational characteristics of pRRd stars are compared with those of 'classical' and 'anomalous' RRd (cRRd, aRRd) stars. The conclusion by Prudil et al. that pRRd stars form a separate group of double-mode pulsators and are not simply very-short-period cRRd stars is confirmed. V1127 Aql and AH Cam are identified as other probable members of the class of pRRd stars.
\end{abstract}

Key words: stars: variables, stars: RR Lyrae, stars: general, stars: horizontal branch, stars: individual, stars: abundances, The Galaxy: bulge

\section{INTRODUCTION}

For most of the 20th century RR Lyrae stars were thought to be pulsating either in the radial fundamental mode (RRab stars) or radial first-overtone mode (RRc stars), often with slowly increasing or decreasing pulsation periods, and sometimes with amplitude and/or frequency modulations (Blazhko effect). Since the discovery that AQ Leo pulsates simultaneously in the fundamental and first-overtone modes (Jerzykiewicz \& Wenzel 1977, Cox, King \& Hodson 1980) more than 3000 double-mode RR Lyrae (RRd) stars have been found in various Galactic and extragalactic environments: in globular clusters (Cox et al. 1980; Cox, Hodson \& Clancy 1983; Sandage, Katem \& Sandage 1981; Nemec 1985b; Clement et al. 1986; Nemec \& Clement 1989), in nearby dwarf galaxies (Nemec 1985a; Kovács 2001a; Cseresnjes 2001; Bernard et al. 2009), in the Magellanic Clouds (Alcock et al. 1997; Nemec, Walker \& Jeon 2009; Soszyński

\footnotetext{
^ E-mail: nemec@camosun.ca (JMN)
}

et al. 2009, 2010, 2016a,b, 2017a), and in the Galactic Bulge and Disk (Soszyński et al. 2011, 2014b, 2017b, 2019).

Most RRd stars are of the 'classical' variety, pulsating simultaneously with a dominant shorter period $\left(P_{\mathrm{S}}\right)$ and a secondary longer period $\left(P_{\mathrm{L}}\right)$. The overall light curves phased with $P_{\mathrm{S}}$ are of relatively low amplitude and roughly sinusoidal with significant scatter about the mean curve; in fact, observed excess scatter is the usual way in which RRd stars are discovered. In period histograms (see, for example, fig. 32 of Nemec 1985a and fig. 2 of Soszyński et al. 2016b) the two periods straddle the long-period side of the period distribution for RRc (first-overtone) stars and the short-period side of the period distribution for RRab (fundamental-mode) stars. Perhaps the strongest argument that $P_{\mathrm{L}}$ is the fundamental-mode period and $P_{\mathrm{S}}$ is the firstovertone period comes from the observed very-narrow range of period ratios, $0.726<P_{\mathrm{S}} / P_{\mathrm{L}}<0.750$, the range amounting to only 3.3 per cent of the mean period ratio. For a given pair of radial modes the period ratio is always strongly constrained by pulsation models.

(C) 2021 RAS 


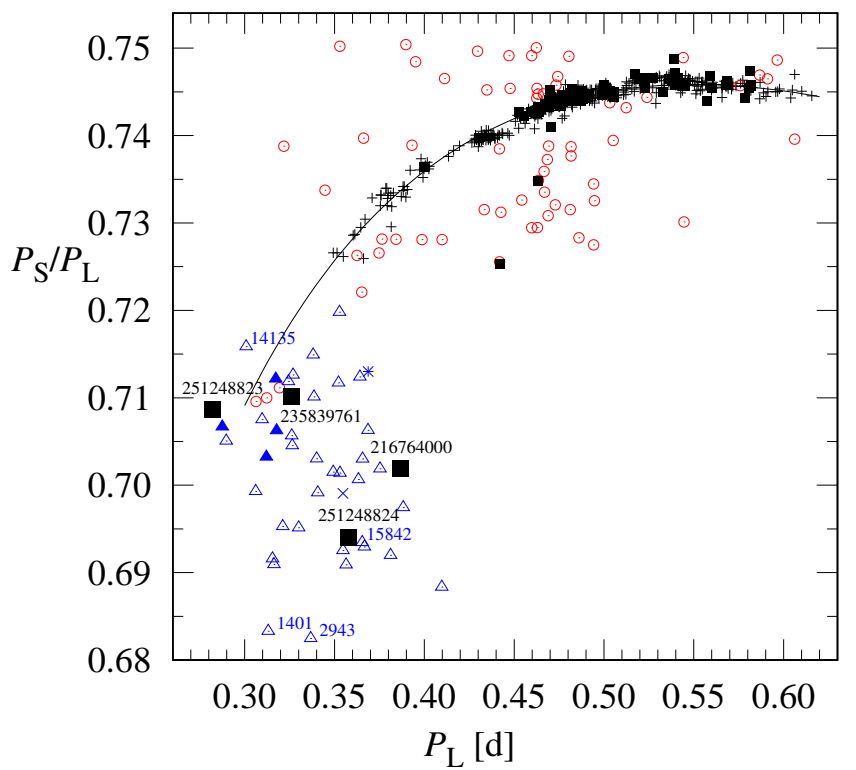

Figure 1. Petersen diagram identifying the four 'peculiar' RRd (pRRd) stars observed by K2 (large black squares labelled with EPIC numbers). Also plotted are the 42 Galactic Bulge pRRd stars studied by Prudil et al. (2017; blue triangles), the Galactic Bulge and Disk RRd stars from Soszyński et al. (2019; black plus signs for 458 classical RRd stars, and red open circles for 63 anomalous RRd stars), and the 76 Ecliptic Plane RRd stars observed by K2 (Nemec, Moskalik et al., in preparation; small black squares). The four pRRd stars with the smallest $A_{\mathrm{S}} / A_{\mathrm{L}}$ amplitude ratios are labelled (in blue) with their OGLE-BLG-RRLYR star numbers (see Fig. 2). The blue filled triangles are the four low-amplitude pRRd stars classified 'RRc' by the OGLE survey. V1127 Aql is represented by the blue cross and AH Cam by a blue asterisk. The mean curve for the 'classical' RRd stars is derived in $\S 4.1$.

In a Petersen (1973) diagram (see Figure 1) the classical RRd (hereinafter 'cRRd') stars trace out a smooth curve, the period ratios decreasing as the periods decrease. The earliest such diagrams revealed that in lowmetallicity Oosterhoff II (OoII) globular clusters, such as M15 and M68 with $[\mathrm{Fe} / \mathrm{H}]=-2.1$ dex (i.e., $Z \sim 0.0001$, assuming $[\mathrm{Fe} / \mathrm{H}]=\log Z+1.9)$, the RRd stars have fundamentalmode periods, $P_{0}\left(=P_{L}\right)$, greater than $0.51 \mathrm{~d}$, and firstovertone periods, $P_{1}\left(=P_{S}\right) \in(0.37 \mathrm{~d}, 0.45 \mathrm{~d})$. And, in less metal poor (i.e., intermediate-metallicity) Oosterhoff I (OoI) globular clusters, such as IC 4499 and M3 with $[\mathrm{Fe} / \mathrm{H}] \sim$ 1.5 dex (i.e., $Z \sim 0.0004$ ), the RRd stars have shorter fundamental-mode periods, $P_{0} \in(0.45 \mathrm{~d}, 0.51 \mathrm{~d})$ and minimum period ratios $\left(P_{1} / P_{0}\right)_{\min }=0.742$ (see, for example, fig. 10 of Clement et al. 1986). These observations clearly established that the location of a cRRd star on the 'classical' curve mainly depends on $[\mathrm{Fe} / \mathrm{H}]$ : the shorter the periods the smaller the period ratio and the higher the metallicity.

In environments consisting of mixtures of stars exhibiting a range of metallicities, such as dwarf galaxies and the Magellanic Clouds (Nemec 1985a; Alcock et al. 1997, 2000; Cseresnjes 2001; Kovács 2001a; Soszyński et al. 2016b), the RRd stars exhibit a wide range of periods and period ratios. In such systems significant differences in the periods of the shortest-period RRd stars are observed. For example, the LMC has a shorter minimum fundamental-mode period, $P_{0, \text { min }}$, and a smaller minimum period ratio, $\left(P_{1} / P_{0}\right)_{\min }$, than the SMC (Soszyński et al. 2010; see fig. 1 of Soszyński et al. 2016b). If metallicity is the main cause of these differences then it follows that the most metal-rich RRd stars in the LMC, those with the smallest period ratios, are more metal-rich than those in the SMC, a result consistent with the LMC having a higher mean metallicity than the SMC (see fig. 2 of Kovács 2009, and fig. 1 of Mucciarelli 2014). Since the shortest-period RRd stars in the LMC also tend to be more centrally concentrated than the less metal-rich RRd stars in the LMC (see figs. 3-4 of Soszyński et al. 2016a) this also argues for a metallicity gradient. In our own Galaxy the most metal-rich RR Lyrae stars (see Soszyński et al. 2019) are more metal-rich than the most metal-rich RR Lyrae stars in the Magellanic Clouds, and have kinematics consistent with membership in the thick- and thin-disk and bulge stellar populations, possibly with quite young ages (Preston 1959, Layden 1995, Kinemuchi et al. 2006, Chadid, Sneden \& Preston 2017, Sneden et al. 2018).

Clear illustrations of how the periods and period ratios of RRd stars depend on mass $(M)$, luminosity $(L)$, effective temperature $\left(T_{\text {eff }}\right)$ and chemical composition are given by Popielski, Dziembowski \& Cassisi (2000, fig. 3) and by Soszyński et al. (2011, fig. 9; 2014a, fig. 2). Several thousand RRd stars are now known, over 2600 of them in the Magellanic Clouds. More than 500 have been identified in our Galaxy, many of which reside in the Galactic Bulge and tend to have shorter periods and smaller period ratios than do the RRd stars located in globular clusters or in the Magellanic Clouds. Varying one or more of the above physical characteristics introduces spread into the distribution of stars about the classical RRd curve. Such variations might help to explain some of the unusual period ratios of the 'anomalous' RRd stars (see below), however, at present they have little bearing on the question of amplitude ratios or the Blazhko effect. Still not well understood is why a particular pulsation mode is dominant (i.e., mode selection), why some RRd stars switch modes on short time-scales (Clement et al. 1997; Soszyński et al. 2014a, 2019), the cause(s) of Blazhko modulations, and the non-linear properties of multi-mode pulsators (e.g., amplitude ratios).

Large-scale surveys and high-precision time-series photometry from the ground and with space telescopes have led to the discovery of several new types of multi-mode RR Lyrae stars (see Moskalik 2014, and Smolec et al. 2017b for recent reviews). The so-called 'anomalous' RRd stars (hereinafter 'aRRd' stars, the notation adopted in the online 'OGLE Collection of Variable Stars') differ from cRRd stars in one or more of the following ways (see Jurcsik et al. 2015; Soszyński et al. 2014b, 2016b, 2017a,b, 2019; Smolec et al. 2015, 2017a): (1) in the Petersen diagram they lie below or above the curve defined by the cRRd stars; (2) in the majority of cases the dominant pulsation mode is the (longer-period) fundamental mode rather than (shorter-period) first-overtone mode, i.e., $A_{1}<A_{0}$; and (3) the Blazhko effect (i.e. long-term modulation of the amplitudes, frequencies and period ratios) is often present, with several Blazhko stars having been observed to switch between double-mode and single-mode RRab pulsation and to have undergone an abrupt change of the fundamentalmode period (e.g., Jurcsik et al. 2015). Sixty-three aRRd 
stars (from Soszyński et al. 2019) are present in Fig. 1 (red open circles); five are triple-mode pulsators; at least one has switched pulsation mode from double-mode (Soszyński et al. 2017b, 2019) to RRab (with Blazhko modulations); and at least three were observed by K2 (Nemec, Moskalik et al., in preparation). To explain the anomalous locations in the Petersen diagram of several RRd stars in the globular cluster M3, the possibilities of mass transfer in a binary system and unusual helium compositions have been considered by Clementini et al. (2004). More recently, Soszyński et al. (2016b) attribute the behaviour to a resonance instability involving the fundamental, first-overtone and second-overtone frequencies (specifically, $2 f_{1} \sim f_{0}+f_{2}$ ).

In Figure 2 amplitude ratios for the stars included in Fig. 1 are plotted against the longer periods. A similar graph, apart from the logarithmic scaling and the labelled groupings, was made by Soszyński et al. (2009) for the RRd stars in the LMC. The longer period is seen to be dominant (i.e., $\left.A_{\mathrm{L}}>A_{\mathrm{S}}\right)$ for all of the pRRd stars, most of the aRRd stars, and for 14 of the 39 short-period cRRd stars, whilst the firstovertone dominates for almost all of the intermediate- and long-period cRRd stars (OoII- and OoI-like) and for 25 of the short-period cRRd stars. The amplitude ratios appear to trend upward as the periods increase, with an apparent discontinuity occuring around $P_{L} \sim 0.45 \mathrm{~d}$, resembling somewhat the jump of $P_{S} / P_{L}$ occuring for the cRRd stars at the same period (see Fig. 1). Figs. 1-2, which are discussed further in $\S 4$, provide valuable observational constraints for models that aim to understand the pulsational and evolutionary states of RRd stars (Kovács 2001b; Kolláth et al. 2002; Szabó, Kolláth \& Buchler 2004; Smolec et al. 2016).

Almost all cRRd stars that have been observed from space have been found to exhibit, in addition to the two main pulsation modes, low-amplitude oscillations with periods $P_{x} \sim 0.61 P_{1}$ (Moskalik et al. 2015; Nemec, Moskalik et al., in preparation). Such period ratios, which are also observed in most RRc stars, can be interpreted as "a manifestation of nonradial mode excitation" (Dziembowski 2012, 2016). Some examples include AQ Leo (Gruberbauer et al. 2007), CoRoT 101368812 (Chadid 2012, Szabó et al. 2014), EPIC 60018653 and 60018662 (Molnár et al. 2015), and EPIC 201585823 (Kurtz et al. 2016).

Other kinds of multi-mode RR Lyrae stars are summarized by Netzel \& Smolec (2019). These include the so-called $\mathrm{R}_{0.61}$ and $\mathrm{R}_{0.68} \mathrm{RRc}$ stars (see Netzel, Smolec \& Moskalik 2015a,b), long-period RRab stars $\left(P_{0}>0.6 \mathrm{~d}\right)$ exhibiting additional shorter-period low-amplitude variability where the period ratios are not unlike those of cRRd and aRRd stars (Smolec et al. 2016), and RRd stars pulsating in the fundamental and second-overtone modes with period ratios $\sim 0.58$ (Benkő et al. 2010, 2014). Amplitude and phase modulations (Blazhko effect) are also present in many RR Lyrae stars (Jurcsik et al. 2009, 2017; Benkő et al. 2014). Recent developments concerning such modulations are discussed by Jurcsik et al. (2018), Kovács (2018a), Kolláth (2018) and Netzel et al. (2018).

Of particular interest for the current paper, Prudil et al. (2017; hereinafter P17) identified 42 doubly periodic RR Lyrae stars that they described as 'peculiar'. The stars were found as a result of reanalyzing 20102013 OGLE-IV photometry for over 38000 Galactic Bulge RR Lyrae stars (Soszyński et al. 2011, 2014b; Udalski,

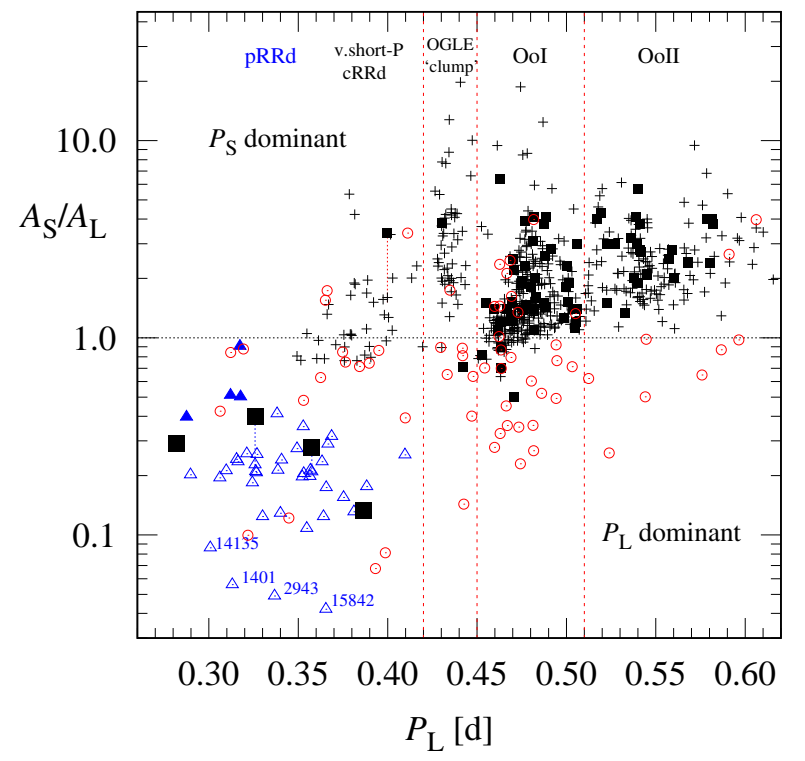

Figure 2. Amplitude ratio $A_{\mathrm{S}} / A_{\mathrm{L}}$ vs. longer period $P_{\mathrm{L}}$ for the RRd stars in Fig. 1 (same symbols). Below $A_{\mathrm{S}} / A_{\mathrm{L}}=1.0$ the longer period is dominant, which is the case for all the pRRd stars and most of the aRRd stars. Except for EPIC 216764000, whose amplitude ratio is based on $K 2$ photometry, the amplitude ratios were derived from Fourier first-term $I$-passband photometry. The short red and blue vertical lines join the OGLE-IV- $I$ and K2-Kp amplitude ratios. For EPIC 251248823 (= OGLE-BLG-RRLYR-00595) the $I$ and $K p$ amplitude ratios are practically identical. The red vertical dashed lines at $P_{L}=0.42 \mathrm{~d}, 0.45 \mathrm{~d}$ and $0.51 \mathrm{~d}$ identify the OGLE 'clump', OoI and OoII stars.

Szymański \& Szymański 2015). Fourteen of the stars had been seen to have secondary pulsations by the OGLE survey but had not been discussed. The 42 'peculiar' RRd (or 'pRRd') stars were found to have dominant pulsation periods $P_{\mathrm{D}} \sim 0.34 \mathrm{~d}$, and shorter additional periods $P_{\mathrm{A}} \sim 0.24 \mathrm{~d}$, with period ratios $P_{\mathrm{A}} / P_{\mathrm{D}}$ between 0.68 and 0.72 . Such period ratios are significantly smaller than the observed period ratios of 'classical' and 'anomalous' RRd stars (see Fig. 1). Since the light curves of the dominant mode for all 42 stars are asymmetric when phased with $P_{D}$ (see fig. 4 of P17), and resemble the light curves of low-amplitude RRab stars, P17 concluded that the dominant (longer-period) pulsation mode most likely is the radial fundamental mode for all 42 stars. The nature of the additional shorter-period pulsation was considered uncertain and of unknown origin, leading P17 even to question whether or not the stars are RR Lyrae stars, that is to say, old low-mass (0.5$\left.0.9 \mathrm{M}_{\odot}\right)$, high-luminosity $\left(30<\mathrm{L} / \mathrm{L}_{\odot}<60\right)$, horizontal branch stars evolving through the Instability Strip with mean surface temperatures $6200<\mathrm{T}_{\text {eff }}<7500 \mathrm{~K}$.

The present paper concerns four such 'peculiar' RRd stars, all located in the direction of the Galactic Bulge and observed during the K2 Mission (Howell et al. 2014). The stars are among $\sim 80 \mathrm{RRd}$ stars that were found when more than 3000 RR Lyrae stars observed by K2 were screened in order to identify and study the RRd stars (Nemec, Moskalik et al., in preparation; see Moskalik et al. 2018a,b for a preliminary report). The brightest of the four stars, EPIC 216764000 (=V1125 Sgr), was observed during K2 
Campaign 7 and is located in the foreground of the Sagittarius dwarf galaxy. It is a newly discovered pRRd star, and although previously observed, the additional pulsation detected in the K2 photometry is a new discovery. The three fainter stars were observed during Campaign 11 and are considerably more distant. When the four stars were first plotted in a Petersen diagram they stood out from the other classical and anomalous RRd stars (see Fig. 1). An overlay of RRd stars in the Galactic Bulge (Soszynski et al. 2011, 2014b, 2019) and the 42 pRRd stars discovered by P17 showed that the three Campaign 11 variables are among the stars studied by P17. The fourth star, EPIC 216764000, almost certainly belongs to his group as well.

\section{PHOTOMETRY}

The main data analyzed were the brightness measurements made with the Kepler telescope (Borucki et al. 2010) during the K2 Mission (Howell et al. 2014). The high precision and relatively-long duration of the Kepler photometry ( $K p$ filter) offers the opportunity for improving our understanding of pRRd stars. The integration times were $29.4 \mathrm{~min}$ (i.e., long cadence), corresponding to a sampling rate of 48.9 measurements per day. Thus the highest discernable frequencies are near the Nyquist frequency, $f_{N}=24.47 \mathrm{~d}^{-1}$, corresponding to minimum periods of $\sim 1 \mathrm{~h}$. The K2 data were subject to continuous drifting of the telescope, with thrusters fired every six hours to maintain pointing. Owing to this instrumental effect the photometry often has an extraneous trend due to the drift and imposed thruster-firing frequencies near $4.08 \mathrm{~d}^{-1}$ and its multiples (seen in Fig. $6 \mathrm{~g}$ ). Once detrended the photometry offers almost continuous brightness measurements with milli-magnitude precision. Since all four stars were observed by $K 2$ for $\sim 11$ weeks the highprecision photometry also offers the opportunity to observe directly cycle-to-cycle light variations. The source files are available at the MAST website (https://archive.stsci.edu).

Data from other surveys undertaken prior to K2 were available for all four stars. These earlier observations provide valuable auxiliary information on such quantities as mean magnitudes and colours, distances, pulsation frequencies at different epochs, high-resolution finding charts, etc. (see $\S 2.3$ ).

\subsection{K2 Campaign 7 photometry}

The K2 observations of EPIC 216764000, the brightest of the four stars, were made in 2015 during Campaign 7, which began on October 4 and ended 81.37 days later on December 26. A total of more than 3700 long-cadence (i.e., 30-min) sequential brightness measurements were made and are shown in Figure 3. The mean magnitude, $\langle K p\rangle=13.741 \mathrm{mag}(\mathrm{Hu}-$ ber et al. 2016), makes EPIC 216764000 the brightest pRRd star yet discovered ${ }^{1}$ and an ideal candidate for spectroscopy.

\footnotetext{
1 The brightest star in the P17 sample is OGLE-BLG-RRLYR-15842 with $I=15.00 \mathrm{mag}$, which is more than two magnitudes fainter than the $I=12.63 \mathrm{mag}$ for EPIC 216764000 .
}
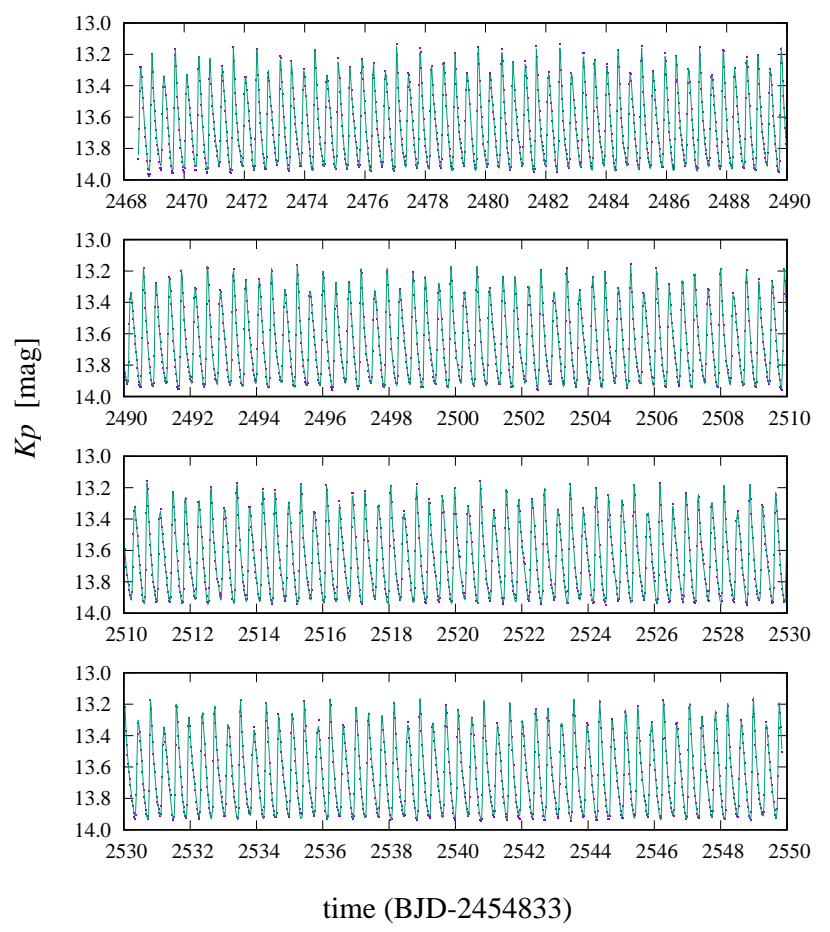

Figure 3. Observed (and fitted) light curve for the 'peculiar' RRd star EPIC 216764000 (=V1125 Sgr = ASAS 191734-2146.2) observed during Campaign 7. The four panels show all 81.37 days of K2 photometry, a total of 3728 long-cadence measurements. The dominant variability is asymmetric, periodic with period $0.38675 \mathrm{~d}$, and clearly exhibits amplitude variability with most of the variation near maximum light.

On the sky it lies in a direction of the Sagittarius constellation that is relatively free of bright stars, with only minor contamination from a faint neighbour 3.8 arcsec to the south-west $2^{2}$

Various approaches were tried to obtain optimallydetrended, flat-field-corrected, outlier-free photometric fluxes. Kurtz et al. (2016) in their analysis of the RRd star EPIC 201585823, recommended that K2-Pixel Photometry (K2P ${ }^{2}$, see Lund et al. 2015; Handberg \& Lund 2017) be used for RR Lyrae stars; however, such detrendings were not made for the Campaign 7 (and 11) data sets.

Our analysis of EPIC 216764000 started with inspection of the 'Pre-search Data Conditioning Simple Aperture Photometry' PDCsap (see Smith et al. 2012) photometric measurements provided by the NASA-Ames Kepler/K2 Science Center. The PDCsap data comprise 3728 flux measurements

2 The Gaia (DR2) equatorial coordinates for EPIC 216764000 and its close neighbour are RA, DEC $=19^{\mathrm{h}} 17^{\mathrm{m}} 33 \mathrm{~s} 344,-21^{\circ} 46^{\prime}$ $02^{\prime \prime} 79$ and $19^{\mathrm{h}} 17^{\mathrm{m}} 33.141,-21^{\circ} 46^{\prime} 05^{\prime \prime} 11$, respectively. The neighbour, fainter than EPIC 216764000 by $\Delta \mathrm{m}_{g}=3.6 \mathrm{mag}$, contributes negligibly to the K2 flux. Gaia (DR2) gives for the parallax $\pi=0.235 \pm 0.031$ mas (see Table 1 ), corresponding to the distance $4.26 \pm 0.57 \mathrm{kpc}$, which is considerably nearer than the $20 \pm 2 \mathrm{kpc}$ distance to the background Sagittarius dwarf galaxy, and roughly half-way to the Galactic Centre. Gaia (DR2) also finds for the effective temperature $T_{\text {eff }}=6135 \mathrm{~K}$, which is $\sim 900 \mathrm{~K}$ smaller than the $7046 \mathrm{~K}$ value given in the EPIC catalog; both values are within the observed range for RR Lyrae stars. 
ranging from 33966 electrons per second (minimum light) to 73552 electrons per second (maximum light). Even better light curves were obtained with the Everest (Luger et al. 2016) and EAP (Plachy et al. 2019) pipelines, and with our own reductions using the Pyke software (Still \& Barclay 2012), all of which gave similar results. Fluxes were transformed to Kepler magnitudes with the Lund et al. (2015) relation, i.e., $K p=25.3-2.5 \log$ (flux), and all the photometric results are based on this transformation.

The K2 photometry for EPIC 216764000 shown in Fig. 3 includes a fitted (Period04) light curve (see $\S 3.1$ below). The dominant period $0.386748 \mathrm{~d}$ is slightly longer than the period $0.3867145( \pm 13)$ d first determined by Uitterdijk (1949), with a trough-to-peak total amplitude $\sim 0.7 \mathrm{mag}$ and an asymmetric light curve shape for each cycle. Cycle-to-cycle amplitude variations are seen with most of the variation occuring at the brightest phases, i.e., the magnitudes at minimum are approximately constant (at $13.95 \mathrm{mag}$ ) while those at maximum light vary from 13.10 to $13.35 \mathrm{mag}$. The mean magnitude derived using the Lund et al. formula, $K p=13.680 \mathrm{mag}$, agrees well with the 13.741 mag given in the EPIC catalog.

\section{$2.2 \quad$ K2 Campaign 11 photometry}

The three pRRd stars observed during Campaign 11, in order of decreasing brightness, are EPIC 235839761, 251248823, and 251248824. All are relatively faint, the brightest being almost four magnitudes fainter than EPIC 216764000, and EPIC 251248823 (=OGLE-BLG-RRLYR-00595) is the shortest-period pRRd star presently known. All were observed in 2016 over 74.2 days (from September 24 to December 8). The Campaign 11 field lies in the direction of the Galactic Bulge and suffers from considerable crowding. Owing to "an error in the initial roll-angle used to minimize solar torque on the spacecraft", and corrective action, a three-day break occurred in the observations (between BJD 2457679 and 2457682), necessitating a division of the data into two subcampaigns, C111 and C112, the former consisting of 1060 long-cadence measurements and the latter 2188 long-cadence measurements.

After evaluating several different reduction pipelines the PDCsap photometry from the NASA-Ames K2 pipeline (see https://keplerscience.arc.nasa.gov/pipeline.html) was adopted for all three stars. In general the mean levels of the C111 fluxes were found to be smaller by about 10 per cent than the fluxes for the C112 fluxes, and for EPIC 251248823 the PDCsap C111 data show an upward trend. To correct for these effects it was necessary to level the fluxes by fitting polynomials and then to bring the C111 fluxes up to the $\mathrm{C} 112$ levels (after removing $5 \sigma$ outliers and other points flagged in the headers as possibly in error) by multiplying by an appropriate constant. In this way the mean flux levels of the optimum photometry for the three stars were found to be 1212, 163 and 70 electrons per second. After transforming the observed fluxes to $K p$ magnitudes using the Lund et al. (2015) formula, the derived mean $K p$ magnitudes were found to be $17.59,19.77$ and $20.69 \mathrm{mag}$, respectively, all of which are significantly fainter than the EPIC catalog (Huber et al. 2016) values: $16.90,18.80$ and $19.80 \mathrm{mag}$.

To resolve these differences the mean magnitudes given in the OGLE and Gaia catalogs were considered. The mean
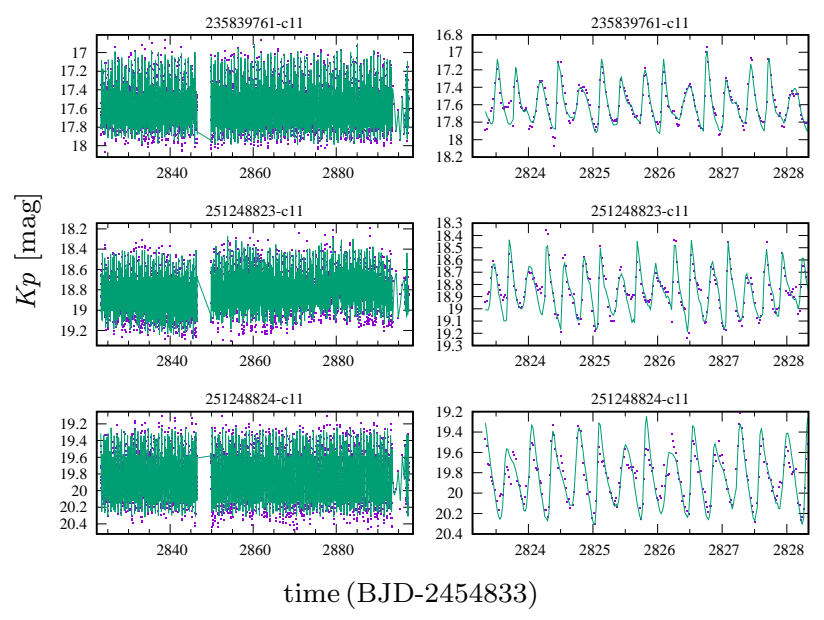

Figure 4. Observed (and fitted) light curves for the three 'peculiar' RRd stars observed during Campaign 11. The left panels show all the K2 photometry, and the right panels show the first five days of data. The observations before time $=2848$ are from the aborted data segment ' $\mathrm{C} 111$ ' and the later observations are from the corrected data segment ' $\mathrm{C} 112$ '. The photometry (purple points) is PDCsap data with additional detrending and the fitted light curves (green) are from Period04 analyses.

$V$ magnitudes given in the latest OGLE-IV catalog are 17.51, 18.84 and 19.87 (Soszyński et al. 2017b), respectively, and the $m_{\mathrm{bp}}$ magnitudes given in the Gaia (DR2) catalog are $17.64 \pm 0.06,18.94 \pm 0.08$, and $19.65 \pm 0.09$. Note that for each star the magnitudes are similar. Recalling also that the mean $V$ magnitudes for the non-Blazhko RR Lyrae stars observed during the original Kepler Mission are usually within 0.2 mag of the mean $K p$ magnitudes (see Nemec et al. 2011) suggested that the measured $K p$ magnitudes should be similar to these OGLE-IV and Gaia magnitudes.

For EPIC 235839761 the measured mean $K p=17.59 \mathrm{mag}$ (PDCsap+Lund transformation) is consistent with the OGLE-IV and Gaia magnitudes but not with the brighter EPIC value $(K p=16.90 \mathrm{mag})$. For this reason the EPIC mean magnitude was rejected in favour of the magnitudes derived using Equation 2.1 of Lund et al. (2015). For EPIC 251248823 the derived mean ( $K p=19.77 \mathrm{mag}$ ) was found to be a full magnitude fainter than the EPIC, OGLE and Gaia means ( $K p=18.80 \mathrm{mag}, V=18.84 \mathrm{mag}$, $\left.m_{\text {bp }}=18.94 \mathrm{mag}\right)$ which are all consistent; so for it the PDCsap mean flux level was increased (by multiplication) from 163 to 400 electrons per second (corresponding to $K p=18.8 \mathrm{mag}$ ). For EPIC 251248824, since the EPIC, OGLE-IV and Gaia catalogues consistently give $K p \sim 19.8 \mathrm{mag}$, the unrealistic $K p=20.69 \mathrm{mag}$ was rejected in favour of normalizing the fluxes so that $K p \sim 19.8 \mathrm{mag}$. The resulting time-series are shown in Figure 4. Apart from minor changes to Fourier amplitudes, these adjustments had little effect on the results of the frequency analyses discussed below (§3).

\subsection{Other observations}

Tables 1-2 summarize much of what was known prior to K2 about EPIC 216764000 and the three fainter Campaign 11 stars. Apart from the Uitterdijk (1949) results, most of 
Table 1. Previous observations of EPIC 216764000

\begin{tabular}{|c|c|}
\hline $\begin{array}{c}\text { Observable } \\
(1)\end{array}$ & $\begin{array}{l}\text { Value } \\
(2)\end{array}$ \\
\hline $\begin{array}{l}\text { RA (J2000) } \\
\text { DEC (J2000) } \\
\text { RA (J2000) } \\
\text { DEC (J2000) } \\
\text { Galactic longitude (J2000), } l \\
\text { Galactic latitude }(\mathrm{J} 2000), b\end{array}$ & $\begin{array}{c}19^{\mathrm{h}} 17^{\mathrm{m}} 33^{\mathrm{s}} \cdot 34375 \\
-21^{\circ} 46^{\prime} 02^{\prime \prime} .7859 \\
289.388932 \\
-21.767441 \\
15.9433 \\
-15.2531\end{array}$ \\
\hline $\begin{array}{l}\text { Johannesburg plates (1928-1937): Uitterdijk } \\
\text { Identification number (van Gent) } \\
\text { No. photographic plates (epoch) } \\
\text { Period, } P \text { ( } \pm \text { mean error) [d] } \\
\text { Time of max. light }( \pm \text { mean error }), t_{0} \text { [BJD] } \\
\Delta \text { phase (= risetime, RT) }\end{array}$ & $\begin{array}{c}130(38) \\
334(1928-1937) \\
0.3867145( \pm 13) \\
2426568.308( \pm 3) \\
0.20\end{array}$ \\
\hline $\begin{array}{l}\text { EPIC Catalog: Huber et al. (2016) } \\
\text { Identification number } \\
\text { Kepler magnitude, } K p[\mathrm{mag}] \\
\text { UCAC4 proper motion }(\mathrm{RA}), \mu_{\alpha}\left[\mathrm{mas} \mathrm{yr}^{-1}\right] \\
\text { UCAC4 proper motion }(\mathrm{DEC}), \mu_{\delta}\left[\mathrm{mas} \mathrm{yr}^{-1}\right] \\
B[\mathrm{mag}] \\
V \text { [mag] } \\
g \text { [mag] } \\
r \quad[\mathrm{mag}] \\
i \quad[\mathrm{mag}] \\
\text { Effective temperature, } T_{\text {eff }}[\mathrm{K}] \\
\text { Surface gravity, log } g[\mathrm{cgs}] \\
\text { Metal abundance, }[\mathrm{Fe} / \mathrm{H}][\mathrm{dex}] \\
\text { Radius, R/R } \odot \\
\text { Mass, M/M } \odot \\
\text { Density, } \rho[\mathrm{g} \mathrm{cm} \\
\text { Distance }[\mathrm{pc}] \\
\text { Reddening, } E(B-V)[\mathrm{mag}]\end{array}$ & $\begin{array}{c}216764000 \\
13.741 \\
5.6 \pm 2.2 \\
1.0 \pm 3.7 \\
14.20 \pm 0.34 \\
13.79 \pm 0.32 \\
13.91 \pm 0.41 \\
13.69 \pm 0.18 \\
13.48 \pm 0.30 \\
7046 \\
4.11 \\
+0.098 \\
1.797 \\
1.582 \\
0.257 \\
1400 \\
0.227\end{array}$ \\
\hline $\begin{array}{l}\text { ASAS-3 Survey (2001-2009): Szczygieł et al. (2 } \\
\text { Identification } \\
\text { Type of variable star } \\
\text { Period, } P \text { [d] } \\
\text { Time of maximum light, } t_{0}[\mathrm{BJD}] \\
\text { Total amplitude, } A_{\mathrm{tot}}(V), A_{\mathrm{tot}}(I)[\mathrm{mag}] \\
V \text { min, max, avg }[\mathrm{mag}] \\
I \text { min, max, avg }[\mathrm{mag}] \\
{[\mathrm{Fe} / \mathrm{H}] \mathrm{JKZW}[\mathrm{dex}]} \\
{[\mathrm{Fe} / \mathrm{H}] \mathrm{S} 04[\mathrm{dex}]}\end{array}$ & $\begin{array}{l}\text { 9) } \\
\text { 191734-2146.2 } \\
\text { RRab } \\
0.3867075 \\
2452026.41 \\
0.439,0.299 \\
13.523,13.104,13.369 \\
12.712,12.522,12.626 \\
0.14 \pm 0.31 \\
0.50\end{array}$ \\
\hline $\begin{array}{l}\text { 2MASS Survey: Skrutskie } \\
\text { Identification } \\
J \text { [mag] } \\
H \text { [mag] } \\
K \text { [mag }]\end{array}$ & $\begin{array}{c}\mathrm{J} 19173334-2146027 \\
12.749 \pm 0.023 \\
12.601 \pm 0.027 \\
12.524 \pm 0.029\end{array}$ \\
\hline $\begin{array}{l}\text { Pan-STARRS (DR1) Survey: Chambers et al. (2 } \\
\text { g [mag] } \\
\text { r [mag] } \\
\text { i [mag] } \\
\text { z [mag] }\end{array}$ & $\begin{array}{c}14.1612 \pm 0.1087 \\
13.6603 \\
13.4406 \\
13.6128 \pm 0.0064\end{array}$ \\
\hline $\begin{array}{l}\text { WISE mid-IR Survey: Gavrilchenko et al. (201 } \\
\text { No. } \\
\text { Identification } \\
\text { W1 [mag] } \\
\text { W2 [mag] } \\
\text { W3 [mag] } \\
\text { W4 [mag] }\end{array}$ & $\begin{array}{c}2973 \\
\mathrm{~J} 191733.34-214602.9 \\
12.593 \pm 0.026 \\
12.604 \pm 0.030 \\
12.284 \pm 0.383 \\
8.778\end{array}$ \\
\hline $\begin{array}{l}\text { SuperWASP Survey (2006-2008): Pollacco et al } \\
\text { Identification } \\
\text { m(WASP) [mag] } \\
\text { Period, } P \text { [d] }\end{array}$ & $\begin{array}{l}\text { J196) } \\
\text { J1731.08-214641.1 } \\
13.8336 \\
0.38671815\end{array}$ \\
\hline $\begin{array}{l}\text { Gaia (DR2) Survey: Brown et al. (2018); Cle } \\
\text { Identification number } \\
m_{\mathrm{g}}[\mathrm{mag}] \\
m_{\mathrm{bp}}[\mathrm{mag}] \\
m_{\mathrm{rp}}[\mathrm{mag}] \\
\text { Colour, bp_rp [mag] } \\
\text { Effective temperature, } T_{\text {eff }}[\mathrm{K}] \\
\text { Parallax, } \pi \text { [mas] } \\
\text { Proper motion (RA), } \mu_{\alpha}\left[\text { mas yr }^{-1}\right] \\
\text { Proper motion (DEC), } \mu_{\delta}\left[\text { mas yr }^{-1}\right] \\
\end{array}$ & $\begin{array}{l}\text { ntini et al. (2019) } \\
4081034120298848256 \\
13.782 \pm 0.022 \\
14.099 \pm 0.072 \\
13.387 \pm 0.051 \\
0.711 \\
6135 \pm 130 \\
0.235 \pm 0.031 \\
2.155 \pm 0.048 \\
-2.899 \pm 0.041 \\
\end{array}$ \\
\hline
\end{tabular}

the information comes from large-scale surveys: the Ecliptic Plane Input Catalog (EPIC) ${ }^{3}$ (Huber et al. 2016); the Optical Gravitational Lensing Experiment (OGLE) surveys ${ }^{4}$ (see

3 https://archive.stsci.edu/k2/epic/search.php

4 http://ogledb.astrouw.edu.pl/ogle/OCVS
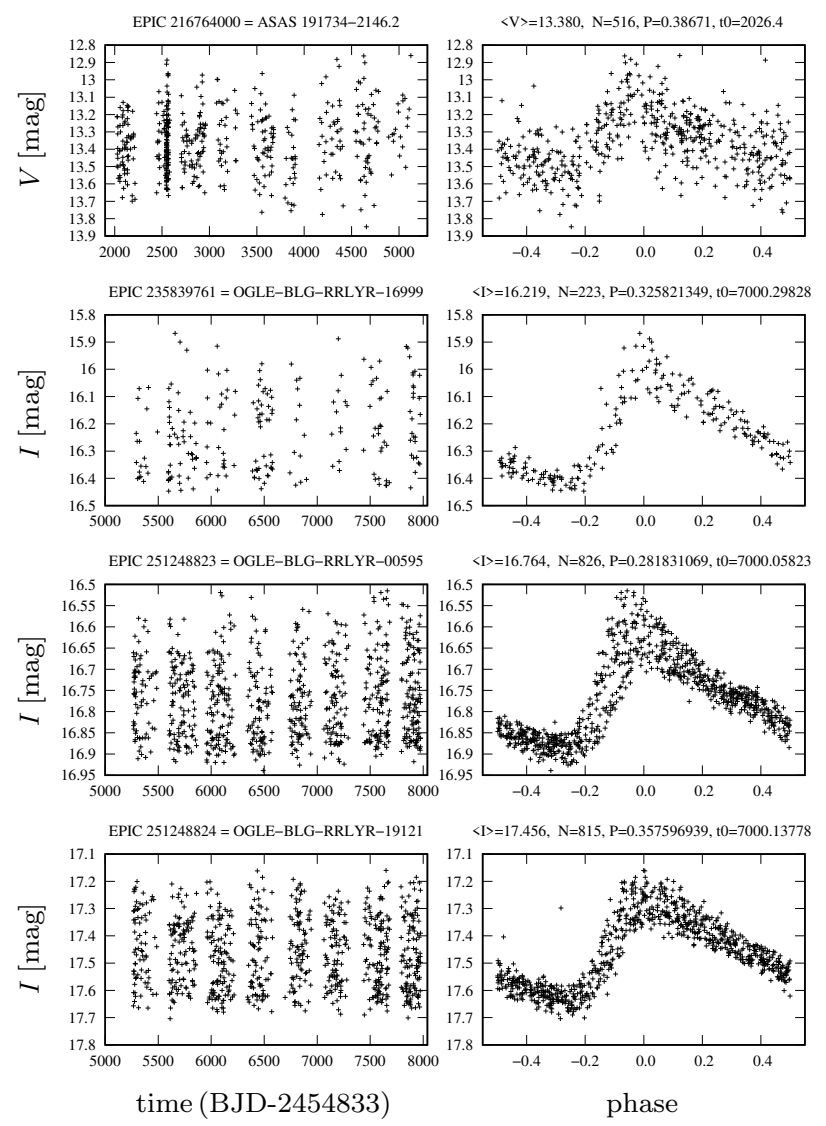

Figure 5. Light curves reproduced from previous ground-based photometry: (Top row) ASAS $V$-photometry for EPIC 216764000; (Bottom rows) OGLE-IV I-photometry (2010-2017) for the three fainter Campaign 11 stars. The left panels show the photometry as a function of time, and the right panels show phased light curves (all asymmetric).

Udalski et al. 2008, 2015; Soszyński et al. 2014b, 2017b); the All-Sky Automated Survey (ASAS) ${ }^{5}$ (Pojmański 1997; Pojmański \& Maciejewski 2005), and the Gaia survey ${ }^{6}$ Other surveys considered here are the 2MASS survey 7 , the Pan-STARRS survey ${ }^{8}$ the WISE survey ${ }^{9}$ and the SuperWASP survey 10 Particularly relevant are the periods and amplitudes reported in P17, where the three Campaign 11 stars were among the $42 \mathrm{RRd}$ stars identified as 'peculiar' (triangles in Fig. 1). Finding charts for all four stars are available through the Aladin Sky Atlas website, the most informative being the high-resolution colour figures formed from Pan-STARRS $g, z$ images.

\subsubsection{Previous photometry of EPIC 216764000}

The variability of EPIC 216764000 was discovered by Hendrik van Gent in the 1930's. Subsequently, 334 photographic

\footnotetext{
5 www.astrouw.edu.pl/asas/

6 https://www.cosmos.esa.int/web/gaia/home

7 https://www.ipac.caltech.edu/2mass/

8 https://panstarrs.stsci.edu/

9 https://www.nasa.gov/mission_pages/WISE

10 https://en.wikipedia.org/wiki/Wide_Angle_Search_for_Planets
} 
Table 2. Previous observations for the three 'peculiar' RRd stars observed by K2 during Campaign 11.

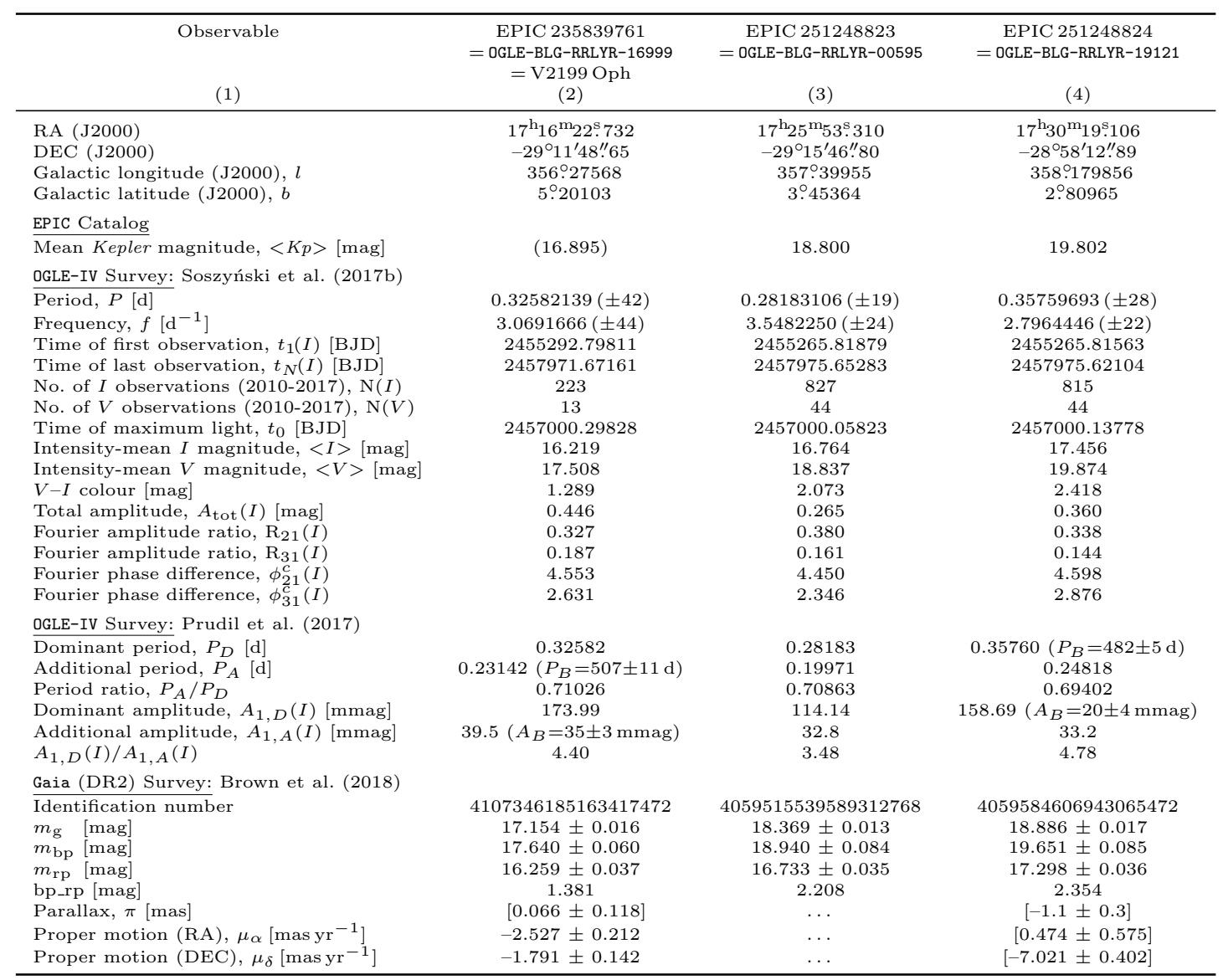

plates taken by van Gent at the Union Observatory (Johannesburg) between 1928 and 1937 were measured by Uitterdijk (1949) who identified it as an RR Lyrae star with pulsation period $0.3867145( \pm 13)$ d. The light curve was shown to be asymmetric (risetime 0.20), with apparent brightness ranging from 13.4 to $14.3 \mathrm{mag}$, and time (HJD) of maximum light $t_{0}=2426568.308( \pm 3)$.

More recently over 500 brightness measurements were made between 2001 and 2009 by the ASAS-3 survey. According to that survey, $P=0.3867075 \mathrm{~d}$, which is consistent with the pulsation period derived by Uitterdijk. No mention was made of amplitude modulation or 'additional' periods. It also is not among the RRd stars in the Galactic Plane identified by Szczygieł \& Fabrycky (2007). However, it was included among the 1455 Galactic RRab stars studied by Szczygieł, Pojmański \& Pilecki (2009), whose paper is the source of most of the ASAS properties in Table 1, including a derived photometric metallicity, $[\mathrm{Fe} / \mathrm{H}]=0.14 \pm 0.31$ dex. In Figure 5 (top panel) a phased light curve reproduced from the ASAS $V$-photometry (assuming $P$ and $t_{0}$ given in the ASAS catalog) is shown. The asymmetry of the light curve, hence the classification as an 'ab-type' RR Lyrae star, is consistent with the findings of Uitterdijk. A reanalysis of the ASAS photometry confirms the basic information given in the ASAS catalog. The pulsation period given there is $0.000040 \mathrm{~d}$ shorter than the 'dominant' period derived from the later $K 2$ data, $P_{D}=0.386748 \mathrm{~d}$ (see below), suggesting the possibility of an increasing dominant period. However, despite trying different period search methods additional periodicity was not detectable in the ASAS data.

EPIC 216764000 was also observed by the SuperWASPSouth cameras over 5600 times between 2006 May 13 and 2008 May 17 (see Butters et al. 2010). From these data the dominant period was estimated to be $P=0.38671815 \mathrm{~d}$ (Greer et al. 2017). Unfortunately, significant gaps are present in the time-series, and the uncertainties in the individual measurements are large, on average $\pm 0.11 \mathrm{mag}$. Consequently, neither the 'additional' period nor amplitude variations were detected. A reanalysis of the SuperWASP data using PDM2 found $P=0.386721 \mathrm{~d}$ (with $\theta_{\min }=0.192$ ) but no detection of the additional period.

\subsubsection{Previous photometry of the Campaign 11 stars}

The most extensive photometric observations of the three fainter stars have been made by the OGLE survey (see Table 2), mainly through $I$ filters (for periods and light curve information), but also through $V$ filters (for colour information). All three stars were observed from 2010-2017 by the OGLE-IV survey (Soszyński et al. 2011, 2017b), and EPIC 251248823 was also observed by the OGLE-III (200104) survey (Udalski et al. 2008). Despite having relatively short (dominant) periods, between $0.28 \mathrm{~d}$ and $0.36 \mathrm{~d}$, and relatively low total amplitudes, $0.26-0.45 \mathrm{mag}$, all three stars were identified by the OGLE classifier as RRab stars, a classification consistent with all three stars having asymmetric 
light curves when the photometry is phased with the dominant period (see Fig. 5).

For EPIC 251248824 (=OGLE-BLG-RRLYR-19121) a secondary period, $P_{A}=0.248191 \mathrm{~d}$, was detected by the OGLE-IV pipeline (see the 'OGLE Collection of Variable Stars' website at http://ogledb.astrouw.edu.pl/ ogle/OCVS/). This period agrees with the 'additional' period derived by P17 from their analysis of the same $I$-photometry and confirmed by the K2 data. In fact, eight of the 42 stars in the original pRRd sample are noted in the OGLE catalogue as having secondary periods similar to those reported by $\mathrm{P} 17$.

P17 also found that OGLE-BLG-RRLYR-16999 and OGLE-BLG-RRLYR-19121 have modulated amplitudes. For the former star (=EPIC 235839761) the additional mode has a modulation (Blazhko) period $P_{B}=507 \pm 11 \mathrm{~d}$, with $I$-amplitude $35 \pm 3 \mathrm{mmag} ;$ and for the latter (=EPIC 251248824) the dominant mode has $P_{B}=482 \pm 5 \mathrm{~d}$, with $I$-amplitude $20 \pm 4 \mathrm{mmag}$ (see discussion in $§ 3.2 .3$ below).

\section{FREQUENCY ANALYSIS}

Detailed frequency analyses of the K2 photometry were conducted for each of the four pRRd stars using several different methods. Initial estimates of the frequencies were made using the Period04 program (Lenz \& Breger 2005), the SigSpec/Combine programs (Reegen 2007), and the FNPEAKS period-searching code (written by Z. Kolaczkowsk: ${ }^{11}$ see Moskalik \& Kołaczkowski 2009). These algorithms employ successive prewhitenings of the data, which were applied manually in the case of Period04 and FNPEAKS and automatically for SigSpec. At each step a multi-frequency model, which includes all the previously identified frequencies, was fitted to the observations. The stopping criterion for the Period04 and FNPEAKS analyses was a signal-to-noise ratio of four for the highest remaining peak in the periodogram (Breger et al. 1993). The SigSpec runs were stopped when the Sig index reached the default value of five (Reegen 2011). Monte Carlo simulation (Period04) was also used to estimate errors for the 'dominant' and 'additional' pulsation frequencies. In addition to these parametric methods, non-parametric period searches were conducted using the Phase Dispersion Minimization (PDM2) method of Stellingwerf $(1978,2011)$. All of these approaches were useful in establishing the significant frequencies present in the data, and in most cases produced consistent results.

Optimized estimates of the frequencies, amplitudes and phases were made using our own non-linear least-squares (Levenberg-Marquardt) multi-frequency fitting program, written in SAS and utilizing its PROC NONLIN procedure. The two-frequency fitting formula was

11 Source available from http://helas.astro.uni.wroc.pl/ deliverables.php?lang $=$ en\&active $=$ fnpeaks

$$
\begin{gathered}
m(t)=m_{0}+\sum_{i=1}^{N_{D}} A_{\mathrm{i}, \mathrm{D}} \sin \left(i \omega_{D}\left[t-t_{0}\right]+\phi_{\mathrm{i}, \mathrm{D}}\right)+ \\
\sum_{j=1}^{N_{A}} A_{\mathrm{j}, \mathrm{A}} \sin \left(j \omega_{A}\left[t-t_{0}\right]+\phi_{\mathrm{j}, \mathrm{A}}\right)+ \\
\sum_{i=1}^{N_{D}} \sum_{j=1}^{N_{A}}\left[A_{\mathrm{i}, \mathrm{j}}^{+} \sin \left(\left(i \omega_{D}+j \omega_{A}\right)\left[t-t_{0}\right]+\phi_{\mathrm{i}, \mathrm{j}}^{+}\right)+\right. \\
\left.A_{\mathrm{i}, \mathrm{j}}^{-} \sin \left(\left(i \omega_{D}-j \omega_{A}\right)\left[t-t_{0}\right]+\phi_{\mathrm{i}, \mathrm{j}}^{-}\right)\right],
\end{gathered}
$$

where $m_{0}$ is the mean magnitude, $\omega_{D}$ and $\omega_{A}$ are the angular frequencies for the 'dominant' and 'additional' modes, the $A$ and $\phi$ are the amplitudes and phases of the various terms in the Fourier sums, up to $N_{D}$ terms for the dominant mode and $N_{A}$ terms for the additional mode. The assumed $N_{D}$ and $N_{A}$ values were adjusted to include all significant harmonic peaks in the amplitude spectra.

The derived amplitudes and phases were used to calculate the Fourier decomposition parameters introduced by Simon \& Lee (1981): amplitude ratio $R_{\mathrm{i} 1}=A_{i} / A_{1}$, and epoch-independent phase difference $\phi_{\mathrm{i} 1}=\phi_{i}-i \phi_{1}$, where $i$ denotes the $i$ th harmonic. Superscripts 's' or 'c' on the $\phi_{\mathrm{i} 1}$ parameter will be used to indicate the use of either a sine or cosine series for the Fourier summations.

\subsection{EPIC 216764000 (= V1125 Sgr)}

\subsubsection{Frequencies, Amplitudes and Phases}

Fourier amplitude spectra for EPIC 216764000 are shown in Figure 6 . The 32 most significant frequencies present in the K2 data are summarized in Table 3, which also contains the derived Fourier amplitudes and phases. The highest peak in the top panel corresponds to the dominant frequency, $f_{D}=2.585663( \pm 3) \mathrm{d}^{-1}$, for which the signal-to-noise ratio $(\mathrm{S} / \mathrm{N})$ is $\sim 120$ and the amplitude of the first term of the Fourier sum is $A_{1, D}=285.4 \pm 0.2 \mathrm{mmag}$. The corresponding period, $P_{D}=0.386748( \pm 1) \mathrm{d}$, is slightly longer than the periods determined by the earlier surveys but is consistent with other estimates based on analysis of the $K 2$ data processed through other pipelines. A total of seven high harmonics were detected, four of which are labelled in Fig. 6(a), indicating asymmetry in the shape of the underlying light curve.

After prewhitening with $f_{D}$ and its harmonics, the additional frequency at $f_{A}=3.68369( \pm 3) \mathrm{d}^{-1}$ $\left(P_{A}=0.271468( \pm 2) \mathrm{d}, \quad \mathrm{S} / \mathrm{N}=15.7\right) \quad$ is clearly identified in Fig. 6(b). The main effect of this low-amplitude ( $\left.A_{1, A}=37.8 \pm 0.2 \mathrm{mmag}\right)$ oscillation is to beat with the dominant oscillation, leading to variations of the total amplitude (see Fig. 3). Note that the harmonics at $2 f_{D}$ and $3 f_{D}$ have more power than the additional-mode pulsation. The only detectable harmonic of $f_{A}$ was the first one at $2 f_{A}=7.36738 \mathrm{~d}^{-1}$ (amplitude $1.8 \pm 0.2 \mathrm{mmag}$ ). The peak at the next harmonic, at $3 f_{A}=11.05 \mathrm{~d}^{-1}$, is hidden in the noise and cannot be detected. Also prominent in Fig.6(b) are many frequencies that are linear combinations of $f_{A}$ and $f_{D}$. Their presence argues strongly that both frequencies originate in EPIC 216764000 and do not result from blending with another star. The K2 thruster-firing frequency 

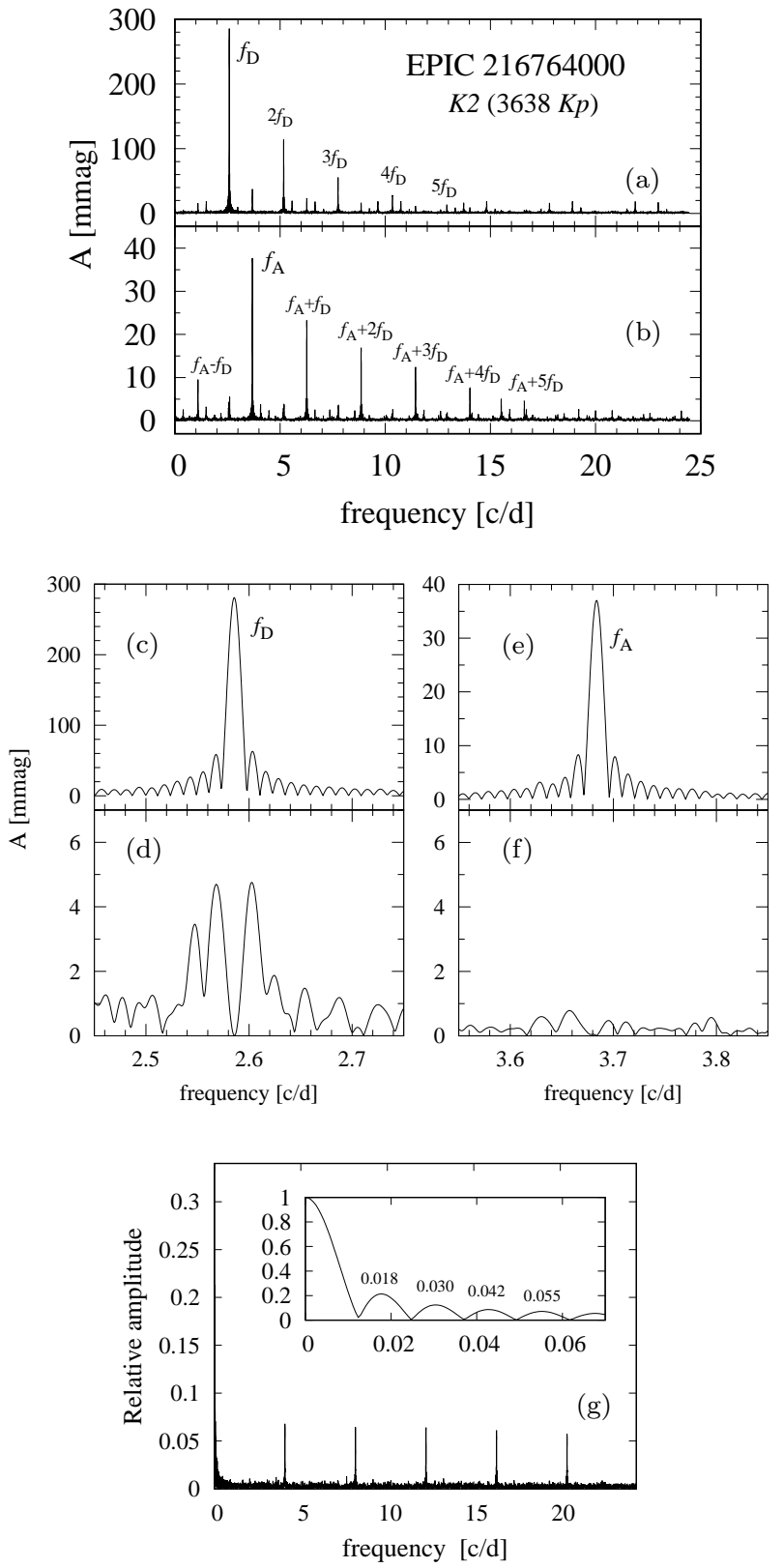

Figure 6. Fourier amplitude spectra for EPIC 216764000 (K2 photometry, EAP pipeline, Period04). (a) The highest peak corresponds to the dominant frequency, $f_{D}=2.585663( \pm 39) \mathrm{d}^{-1}$, for which the first term in the Fourier expansion has an amplitude $285.4 \pm 0.2 \mathrm{mmag}$. The four most powerful of the seven detected harmonics (see Table 3) have been labelled. (b) After prewhitening with $f_{D}$ and its harmonics the highest remaining peak is that of the additional frequency, $f_{A}=3.683689( \pm 43) \mathrm{d}^{-1}$ (amplitude $37.8 \pm 0.2 \mathrm{mmag})$. The other strong peaks are combination frequencies involving $f_{A}$ and $f_{D}$. The harmonic at $2 f_{A}$ is visible but weak (amplitude $1.8 \mathrm{mmag}$ ). (c) Close-up of amplitude spectrum near $f_{D}$. (d) Close-up of spectrum after prewhitening with $f_{D}$ and its harmonics. (e) Close-up of amplitude spectrum in the vicinity of $f_{A}$. (f) Amplitude spectrum after prewhitening with $f_{A}$ and its harmonics. (g) Window function for the K2 photometry.
Table 3. EPIC 216764000 (=V1125 Sgr): frequencies, amplitudes and phases derived from the K2 photometry. The independent frequencies, $f_{D}$ and $f_{A}$, have been underlined, and the uncertainties in the amplitudes and phases are random errors (i.e., do not include systematic errors). The quantity Sig is the significance of the peak in the amplitude spectrum (see Reegen 2007, 2011). The mean magnitude, $\langle K p\rangle=13.7412 \pm 0.0001 \mathrm{mag}$, agrees well with the EPIC mean. The assumed time of maximum light (for the phases) was $t_{0}=$ BJD $2454833.0000+2468.9335$.

\begin{tabular}{|c|c|c|c|c|c|}
\hline Label & $\begin{array}{c}\text { Frequency } \\
\quad\left[\mathrm{d}^{-1}\right]\end{array}$ & $\begin{array}{l}\text { Period } \\
{[\mathrm{d}]}\end{array}$ & $\begin{array}{c}\text { Ampl. } \\
{[\mathrm{mmag}]} \\
\pm 0.20\end{array}$ & $\begin{array}{c}\text { Phase } \\
\text { [rad] }\end{array}$ & Sig \\
\hline \multicolumn{6}{|c|}{ (a) Dominant frequency, $f_{D}(=\mathrm{f} 1)$, and its harmonics } \\
\hline f1 & $\underline{2.58566}$ & 0.386748 & 285.40 & $-2.270 \pm 0.001$ & 690 \\
\hline $2 \mathrm{f} 1$ & $\overline{5.17132}$ & 0.193374 & 113.60 & $-1.797 \pm 0.002$ & 562 \\
\hline $3 \mathrm{f} 1$ & 7.75698 & 0.128916 & 55.06 & $-1.249 \pm 0.004$ & 385 \\
\hline $4 \mathrm{f} 1$ & 10.34264 & 0.096687 & 26.87 & $-0.804 \pm 0.008$ & 269 \\
\hline $5 \mathrm{f} 1$ & 12.92830 & 0.077350 & 13.20 & $-0.131 \pm 0.016$ & 201 \\
\hline $6 \mathrm{f} 1$ & 15.51398 & 0.064458 & 5.47 & $0.572 \pm 0.037$ & 114 \\
\hline $7 \mathrm{f} 1$ & 18.09964 & 0.055250 & 1.65 & $1.132 \pm 0.120$ & 33 \\
\hline $8 \mathrm{f} 1$ & 20.68528 & 0.048344 & 0.49 & $1.449 \pm 0.403$ & 13 \\
\hline \multicolumn{6}{|c|}{ (b) Additional frequency, $f_{A}(=\mathrm{f} 2)$, and its harmonics } \\
\hline f2 & $\underline{3.68369}$ & 0.271468 & 37.81 & $-1.236 \pm 0.008$ & 330 \\
\hline $2 \mathrm{f} 2$ & 7.36738 & 0.135734 & 1.81 & $0.382 \pm 0.111$ & $<5$ \\
\hline \multicolumn{6}{|c|}{ (c) Linear combination frequencies } \\
\hline $\mathrm{f} 1+\mathrm{f} 2$ & 6.26935 & 0.159506 & 23.23 & $-1.142 \pm 0.011$ & 294 \\
\hline $2 \mathrm{f} 1+\mathrm{f} 2$ & 8.85501 & 0.112930 & 17.24 & $-0.818 \pm 0.013$ & 244 \\
\hline $3 \mathrm{f} 1+\mathrm{f} 2$ & 11.44067 & 0.087407 & 12.48 & $-0.333 \pm 0.017$ & 234 \\
\hline $4 \mathrm{f} 1+\mathrm{f} 2$ & 14.02633 & 0.071294 & 7.77 & $0.159 \pm 0.026$ & 163 \\
\hline $5 \mathrm{f} 1+\mathrm{f} 2$ & 16.61199 & 0.060197 & 4.59 & $0.701 \pm 0.044$ & 92 \\
\hline $6 \mathrm{f} 1+\mathrm{f} 2$ & 19.19765 & 0.052090 & 2.62 & $1.291 \pm 0.076$ & 58 \\
\hline $7 \mathrm{f} 1+\mathrm{f} 2$ & 21.78333 & 0.045907 & 1.23 & $1.920 \pm 0.162$ & 27 \\
\hline $8 \mathrm{f} 1+\mathrm{f} 2$ & 24.36897 & 0.041036 & 0.51 & $2.541 \pm 0.389$ & 13 \\
\hline $2 \mathrm{f} 1-\mathrm{f} 2$ & 1.48763 & 0.672210 & 2.88 & $-0.764 \pm 0.069$ & 61 \\
\hline $3 \mathrm{f} 1-\mathrm{f} 2^{a}$ & 4.07273 & 0.245502 & 2.7: & $-0.598 \pm 0.053$ & 58: \\
\hline $4 \mathrm{f} 1-\mathrm{f} 2$ & 6.65895 & 0.150174 & 2.48 & $0.287 \pm 0.080$ & 55 \\
\hline $5 \mathrm{f} 1-\mathrm{f} 2$ & 9.24463 & 0.108171 & 1.23 & $1.694 \pm 0.162$ & 27 \\
\hline $6 \mathrm{f} 1-\mathrm{f} 2$ & 11.83029 & 0.084529 & 0.67 & $1.541 \pm 0.295$ & 14 \\
\hline $7 \mathrm{f} 1-\mathrm{f} 2$ & 14.41595 & 0.069368 & 0.61 & $1.728 \pm 0.323$ & 14 \\
\hline $8 \mathrm{f} 1-\mathrm{f} 2$ & 17.00162 & 0.058818 & 0.56 & $2.453 \pm 0.356$ & 13 \\
\hline $\mathrm{f} 2-\mathrm{f} 1$ & 1.09803 & 0.910725 & 9.72 & $3.088 \pm 0.021$ & 205 \\
\hline $2 \mathrm{f} 2-\mathrm{f} 1$ & 4.78171 & 0.209130 & 0.57 & $1.013 \pm 0.349$ & 13 \\
\hline $\mathrm{f} 1+2 \mathrm{f} 2$ & 9.95304 & 0.100472 & 0.97 & $-0.060 \pm 0.205$ & 25 \\
\hline $2 \mathrm{f} 1+2 \mathrm{f} 2$ & 12.53870 & 0.079753 & 1.15 & $0.581 \pm 0.173$ & 28 \\
\hline $3 \mathrm{f} 1+2 \mathrm{f} 2$ & 15.12436 & 0.066119 & 0.93 & $1.058 \pm 0.214$ & 24 \\
\hline $4 \mathrm{f} 1+2 \mathrm{f} 2$ & 17.71002 & 0.056465 & 0.55 & $1.582 \pm 0.363$ & 14 \\
\hline $5 \mathrm{f} 1+2 \mathrm{f} 2$ & 20.29568 & 0.049272 & 0.37 & $1.771 \pm 0.534$ & 12 \\
\hline
\end{tabular}

Notes: (a) The frequency $3 \mathrm{f} 1-\mathrm{f} 2=4.073 \mathrm{~d}^{-1}$ coincides with the Kepler $/$ K2 thruster-firing frequency, $f_{\mathrm{th}}=4.075 \mathrm{~d}^{-1}$, making measurement of amplitudes and phases of both frequencies highly unreliable.

near $f_{\mathrm{th}}=4.08 \mathrm{~d}^{-1}$ (amplitude $\sim 3 \mathrm{mmag}$ ) is present but coincides with the combination frequency at $3 f_{D}-f_{A}=$ $4.07 \mathrm{~d}^{-1}$, making measurement of amplitudes and phases of both frequencies highly unreliable.

Figs. 6(c)-(f) show close-up views of the amplitude spectra in the vicinity of $f_{D}$ and $f_{A}$. Fig. $6(\mathrm{~d})$ reveals residual power after prewhitening with $f_{D}$ and its harmonics. In general, amplitude and frequency modulations are revealed by sidelobes separated from the main peak by a frequency difference $(\Delta \mathrm{f})$ equal to the reciprocal of the Blazhko period. The sidelobes may be placed symmetrically on the opposite sides of the main peak (equidistant triplets), on one side only (doublets), or occur in multiplets. However in this case the residual peaks with amplitudes $\sim 5 \mathrm{mmag}$, and the next set of sidelobes with amplitudes at $\sim 3$ and 2 mmag are not well resolved from $f_{D}$. They correspond to the wings of the broadened $f_{D}$ left after imperfect prewhitening, i.e., they are related to non-stationarity of $f_{D}$. On the other hand, Fig. 6(f) shows no significant signal left after prewhitening with $f_{A}$ and its harmonic(s), thus there is no evidence in the Fourier transform for non-stationarity of $f_{A}$. Time vari- 

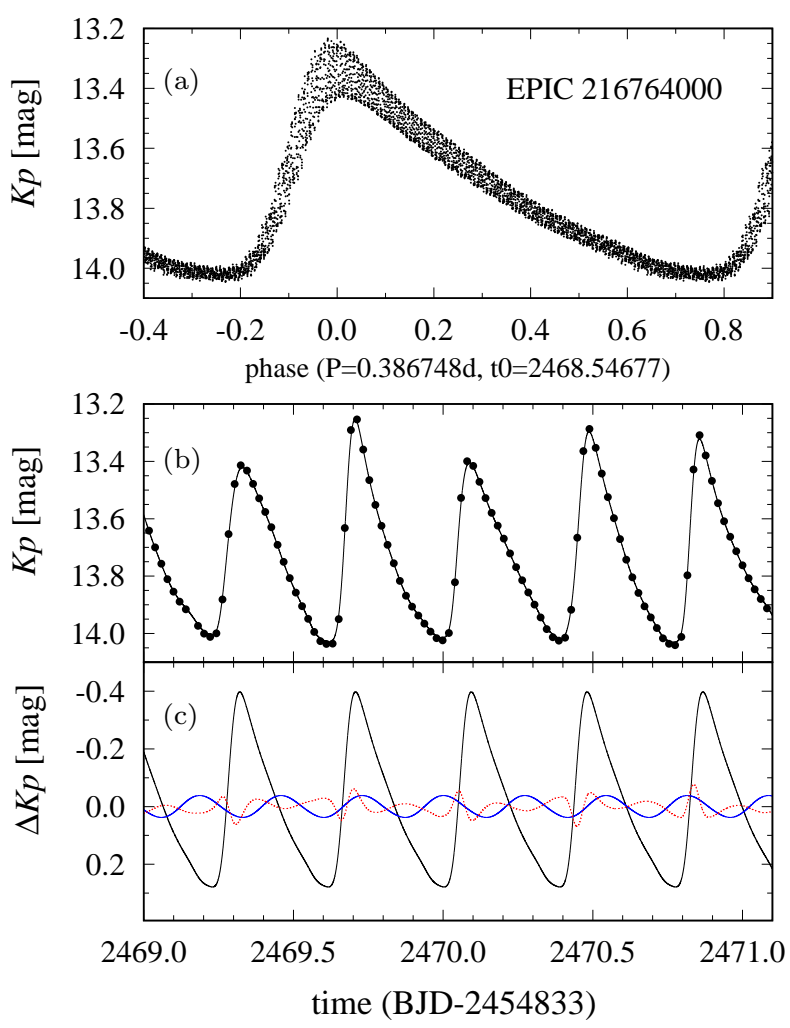

Figure 7. Observed and fitted light curves for EPIC 216764000. (a) Light curve phased with the dominant period (the scatter is due to the unremoved additional oscillation and combination frequencies); (b) Close-up comparison of the fitted and observed K2 light curves ( $\sim 19$ points per cycle); (c) Component light curves derived from non-linear least-squares fitting of the two-frequency model. The lower-amplitude nearly-sinusoidal (blue) curve is for the additional mode with period $P_{A}=0.271468 \mathrm{~d}$, and the dotted (red) curve is the contribution from the combination frequencies.

ations of the amplitudes and frequencies of both $f_{D}$ and $f_{A}$ are investigated further in $\S 3.1 .3$ below.

Fig. 6(g) shows the window function for the K2 photometry, for frequencies out to the long-cadence Nyquist frequency at $24.5 \mathrm{~d}^{-1}$. The constant amplitude spikes at 4.0746 , $8.1573,12.2351,16.3144$ and $20.4996 \mathrm{~d}^{-1}$ are frequencies associated with the K2 telescope thruster firings every six hours. The inset shows the central peak, with measured $\mathrm{FWHM}=0.0148 \mathrm{~d}^{-1}$ (only slightly larger than the Rayleigh criterion $f_{R}=0.0123 \mathrm{~d}^{-1}$ ), and its close sidelobes (labelled with frequency offsets).

\subsubsection{Light curves and Fourier parameters}

Observed and fitted light curves for EPIC 216764000 are plotted in Figure 7 . The top panel shows the observed $K p$ light curve phased with the period $0.386748 \mathrm{~d}$, confirming the asymmetric shape seen by Uitterdijk (1949) and by the ASAS survey (top panel of Fig. 5), and significantly improving upon the definition of the shape. Most of the light variation occurs near maximum brightness. There are no obvious humps on the rise to maximum light, bumps near minimum light (see Christy 1966; Gillett \& Crowe 1988; Chadid et al. 2014), or other such features. The cycle-to-cycle light curve shapes predicted by the two-frequency model (Eqn.1) are
Table 4. Fourier decomposition parameters, risetimes and total amplitudes for both modes of the four pRRd stars observed by K2 and by the OGLE survey. The amplitude units are [mmag]. Note: $\phi_{21}^{c}=\phi_{21}^{s}+\pi / 2$, and $\phi_{31}^{c}=\phi_{31}^{s}-\pi$.

\begin{tabular}{|c|c|c|c|c|}
\hline $\begin{array}{l}\text { EPIC Id: } \\
K p \text { [mag]: } \\
\text { OGLE Id: } \\
\quad(1)\end{array}$ & $\begin{array}{c}216764000 \\
13.74 \\
- \\
(2) \\
\end{array}$ & $\begin{array}{c}235839761 \\
17.59 \\
16999 \\
(3) \\
\end{array}$ & $\begin{array}{c}251248823 \\
18.83 \\
00595 \\
(4)\end{array}$ & $\begin{array}{c}251248824 \\
19.84 \\
19121 \\
(5)\end{array}$ \\
\hline \multicolumn{5}{|c|}{ (a) K2-photometry, Kp-passband (2016) } \\
\hline $\begin{array}{l}f_{D}\left[\mathrm{~d}^{-1}\right] \\
P_{D}[\mathrm{~d}] \\
\mathrm{RT}_{D} \\
A_{\text {tot, }} \\
A_{1, D} \\
R_{21, \mathrm{D}} \\
R_{31, \mathrm{D}} \\
R_{41, \mathrm{D}} \\
R_{51, \mathrm{D}} \\
R_{61, \mathrm{D}} \\
\phi_{21, \mathrm{D}}^{s} \\
\phi_{31, \mathrm{D}}^{s} \\
\phi_{41, \mathrm{D}}^{s} \\
\phi_{51, \mathrm{D}}^{s}\end{array}$ & $\begin{array}{c}2.585663( \pm 3) \\
0.386748( \pm 1) \\
0.235 \pm 0.001 \\
676 \pm 1 \\
285.40 \pm 0.20 \\
0.398 \pm 0.001 \\
0.193 \pm 0.001 \\
0.094 \pm 0.003 \\
0.045 \pm 0.002 \\
0.018 \pm 0.001 \\
2.743 \pm 0.002 \\
5.561 \pm 0.004 \\
1.994 \pm 0.001 \\
4.937 \pm 0.020\end{array}$ & $\begin{array}{c}3.069166( \pm 4) \\
0.325821( \pm 1) \\
0.279 \pm 0.001 \\
627 \pm 3 \\
284.3 \pm 1.5 \\
0.350 \pm 0.008 \\
0.145 \pm 0.006 \\
0.064 \pm 0.006 \\
0.021 \pm 0.006 \\
0.013 \pm 0.006 \\
2.81 \pm 0.03 \\
5.62 \pm 0.06 \\
2.21 \pm 0.11 \\
5.13 \pm 0.29\end{array}$ & $\begin{array}{c}3.548224( \pm 3) \\
0.281831( \pm 1) \\
0.289 \pm 0.001 \\
548 \pm 5 \\
237.7 \pm 2.6 \\
0.370 \pm 0.013 \\
0.169 \pm 0.012 \\
0.083 \pm 0.011 \\
0.032 \pm 0.011 \\
0.023 \pm 0.011 \\
2.57 \pm 0.05 \\
5.20 \pm 0.10 \\
1.74 \pm 0.18 \\
4.21 \pm 0.39\end{array}$ & $\begin{array}{c}2.796445( \pm 2) \\
0.357597( \pm 1) \\
0.277 \pm 0.001 \\
771 \pm 4 \\
345.7 \pm 2.2 \\
0.324 \pm 0.008 \\
0.121 \pm 0.007 \\
0.050 \pm 0.007 \\
0.014 \pm 0.006 \\
\ldots \\
3.04 \pm 0.03 \\
5.93 \pm 0.06 \\
2.64 \pm 0.15 \\
5.86 \pm 0.49\end{array}$ \\
\hline $\begin{array}{l}f_{A}\left[\mathrm{~d}^{-1}\right] \\
P_{A}[\mathrm{~d}] \\
\mathrm{RT}_{A} \\
A_{\text {tot }, \mathrm{A}} \\
A_{1, A} \\
R_{21, \mathrm{~A}} \\
\phi_{21, \mathrm{~A}}^{\mathrm{s}}\end{array}$ & $\begin{array}{c}3.68368( \pm 3) \\
0.271468( \pm 2) \\
0.472 \pm 0.001 \\
76 \pm 2 \\
37.81 \pm 0.20 \\
0.048 \pm 0.005 \\
2.85 \pm 0.11\end{array}$ & $\begin{array}{c}4.32216( \pm 13) \\
0.231366( \pm 7) \\
0.436 \pm 0.002 \\
225 \pm 3 \\
114.1 \pm 1.5 \\
0.12 \pm 0.02 \\
2.71 \pm 0.35\end{array}$ & $\begin{array}{c}5.00709 \\
0.199717 \\
0.421 \pm 0.002 \\
147 \pm 5 \\
69.2 \pm 2.6 \\
0.15 \pm 0.04 \\
3.05 \pm 0.32\end{array}$ & $\begin{array}{c}4.02916 \\
0.248191 \\
0.439 \pm 0.002 \\
198 \pm 4 \\
97.4 \pm 2.2 \\
0.07 \pm 0.02 \\
2.28 \pm 0.37\end{array}$ \\
\hline $\begin{array}{l}A_{\text {tot,D }} \\
R_{21, \mathrm{D}} \\
\phi_{21, \mathrm{D}}^{s} \\
R_{31, \mathrm{D}} \\
\phi_{31, \mathrm{D}}^{\mathrm{s}} \\
R_{21, \mathrm{~A}} \\
\phi_{21, \mathrm{~A}}^{s}\end{array}$ & $\begin{array}{l}\text { (b) OGLE-IV-1 } \\
\ldots \\
\ldots \\
\ldots \\
\ldots \\
\ldots \\
\ldots \\
\ldots\end{array}$ & $\begin{array}{c}\text { tometry, } I \text {-pas } \\
446 \\
0.39 \pm 0.03 \\
2.99 \pm 0.10 \\
0.19 \pm 0.03 \\
5.92 \pm 0.22 \\
0.13 \pm 0.10 \\
2.33 \pm 0.88\end{array}$ & $\begin{array}{c}\text { and }(2010-2017) \\
265 \\
0.39 \pm 0.01 \\
2.91 \pm 0.03 \\
0.18 \pm 0.01 \\
5.55 \pm 0.06 \\
0.13 \pm 0.02 \\
3.14 \pm 0.20\end{array}$ & $\begin{array}{c}360 \\
0.34 \pm 0.02 \\
3.01 \pm 0.06 \\
0.14 \pm 0.02 \\
5.92 \pm 0.15 \\
0.04 \pm 0.06 \\
1.9 \pm 1.4\end{array}$ \\
\hline $\begin{array}{l}\Delta A_{\text {tot, } \mathrm{D}} \\
\Delta R_{21, \mathrm{D}} \\
\Delta \phi_{21, \mathrm{D}}^{s} \\
\Delta R_{31, \mathrm{D}} \\
\Delta \phi_{31, \mathrm{D}}^{s} \\
\Delta R_{21, \mathrm{~A}} \\
\Delta \phi_{21, \mathrm{~A}}^{s}\end{array}$ & $\begin{array}{l} \\
\quad \text { (c) } \mathrm{D} \\
\ldots \\
\ldots \\
\ldots \\
\ldots \\
\ldots \\
\ldots \\
\ldots\end{array}$ & $\begin{array}{c}\text { rences (in the } \\
-181 \\
0.04 \pm 0.03 \\
0.18 \pm 0.10 \\
0.04 \pm 0.03 \\
0.30 \pm 0.23 \\
0.01 \pm 0.10 \\
-0.4 \pm 0.9\end{array}$ & $\begin{array}{c}\text { nse } I-K p) \\
-283 \\
0.02 \pm 0.02 \\
0.34 \pm 0.06 \\
0.01 \pm 0.02 \\
0.35 \pm 0.12 \\
-0.02 \pm 0.04 \\
0.09 \pm 0.38\end{array}$ & $\begin{array}{c}-411 \\
0.02 \pm 0.02 \\
-0.03 \pm 0.07 \\
0.02 \pm 0.02 \\
-0.01 \pm 0.16 \\
-0.03 \pm 0.06 \\
-0.4 \pm 1.4\end{array}$ \\
\hline
\end{tabular}

compared with the observed photometry for the first couple of days of observations in Fig. 7(b). From this figure (and Fig. 3) it is evident that the total amplitude (i.e., min-to$\max ), A_{\text {tot }}$, and to a lesser extent the risetime, RT (see Sandage 2004), change from cycle to cycle. In general the scatter about the fitted light curve is small, $\lesssim 10$ mmag.

Fourier decomposition parameters, mean risetimes and total amplitudes for EPIC 216764000 are given in column (2) of Table 4, where the quantities for the dominant and additional pulsation modes are identified by the subscripts ' $\mathrm{D}$ ' and 'A'. The pulsation period of the dominant mode, $P_{D}=0.387 \mathrm{~d}$, and the asymmetric light curve (characterized by the short risetime, $\mathrm{RT}_{D}=0.235 \pm 0.001$ ), are both consistent with fundamental-mode pulsation in a metal-rich RR Lyrae star (see fig. 4 of Nemec et al. 2013). The short period for the additional mode, $P_{A}=0.271 \mathrm{~d}$, and the long risetime, $\mathrm{RT}_{A}=0.472 \pm 0.001$ (which is only slightly smaller than 0.500 for a pure sinusoid), are both consistent with first-overtone pulsation. The ratio of the total amplitudes, $A_{\text {tot }, D} / A_{\text {tot }, A}=8.89 \pm 0.13$, is significantly larger than the ratio derived using the first term of the Fourier decomposition, $A_{1, D} / A_{1, A}=7.55 \pm 0.09$ (both based on $K p$ magnitudes).

Disentangled light curves for the two modes of EPIC 216764000 are plotted in the top row of Figure 8. The standard deviations of the residuals for the two modes are 8.0 and $2.5 \mathrm{mmag}$, respectively. The light curve for the dominant mode (left panel) was produced by subtracting from 

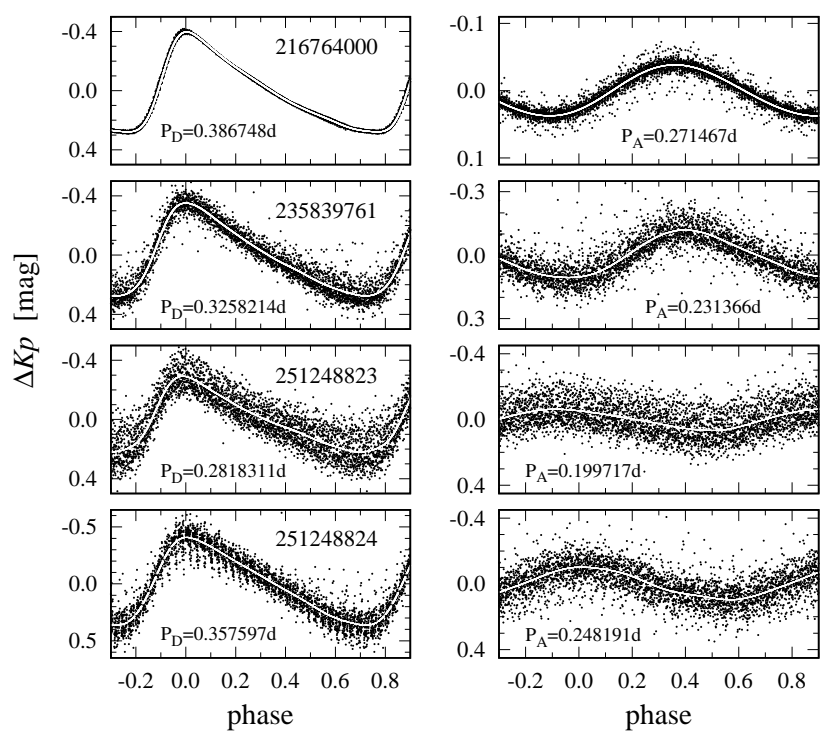

Figure 8. Phased light curves for EPIC 216764000 and the three fainter pRRd stars, revealing the shapes of the dominant and additional modes. Note the different vertical scales for the two modes and for the different stars. The dominant and additional pulsation periods $\left(P_{D}\right.$ and $\left.P_{A}\right)$ are given on each panel. The adopted zero-points (for the phases) were $t_{0}=2454833+$ $2468.93357=2457301.9335$ for EPIC 216764000 , and the OGLE-IV $t_{0}$ values for the three Campaign 11 stars (see Table 2).

the time-series data all the Fourier components (Eqn.1, Table 3 ) related to the additional mode $\left(n f_{A}\right)$ and all the components corresponding to the frequency combinations $\left(\mathrm{m} f_{D} \pm \mathrm{n} f_{A}\right)$, leaving only components related to the fundamental mode, which were then phased with the period of the dominant mode. Because of non-stationarity of both modes (see Fig. 10), the subtraction of unwanted Fourier components was performed with the time-dependent prewhitening method (Moskalik et al. 2015). The light curve of the additional mode (right panel) was produced in a similar way: all components related to the dominant mode $\left(\mathrm{m} f_{D}\right)$ and all components corresponding to the frequency combinations $\left(\mathrm{m} f_{D} \pm \mathrm{n} f_{A}\right)$ were subtracted, and the results phased with the additional period.

\subsubsection{Amplitude and Frequency Variations}

Despite the K2 observations of EPIC 216764000 spanning only $82.6 \mathrm{~d}$, a search was made for possible frequency (or phase) and amplitude variations. In Figure 9 the residual magnitudes (observed minus fitted) are plotted, where the fitted magnitude values were calculated by substituting into Eqn. 1 the parameter estimates given in Table 3. The residuals are clearly not normally distributed but appear to be of two types: low amplitude (from -10 to $+10 \mathrm{mmag}$ ), and higher amplitude (from -40 to $+40 \mathrm{mmag}$ ), the latter starting out negative, turning positive between $t=2501$ and 2531 , and then switching back to negative. If the up-down variations are periodic then the period is $\sim 60 \mathrm{~d}$. The largeamplitude spikes are well resolved (with 3-4 observations per spike) and periodic with a frequency close to $f_{D}$. The spikes are reminiscent of fig. 2 of Kovács (2018b) which shows a similar pattern when the assumed period was purposely set to a value slightly larger than the actual value.

Fig. 9 reveals that the dominant pulsation period is unstable and that $P_{D}$ varies back and forth, which is equivalent to saying that the pulsation phase $\phi_{1, D}$ varies back and forth (see Fig. 10). Because the ascending branch of the light curve (see top left panel of Fig. 8) is much steeper than the descending branch, any phase difference between observed pulsation and the ephemeris (i.e., a horizontal shift) will correspond to a vertical difference which is much larger on the ascending than the descending branch. If the observed maximum happens earlier than the predicted maximum then we will measure large positive residuals (on the ascending branch) and small negative residuals (on the descending branch). It will be the other way around if the observed maximum comes later than the predicted one. This also explains why the spikes repeat with a period close to $P_{D}$.

The fact that the low-amplitude residuals tend to be negative when the high-amplitude residuals are positive is simply a consequence of the asymmetric light curve. The ascending branch of EPIC 216764000 lasts $\sim 0.2 P_{D}$, and the descending branch lasts $\sim 0.8 P_{D}$. Thus, the ascending branch is roughly four times steeper than the descending branch. Consequently we can expect that the highamplitude residuals (coming from the ascending branch) should be roughly four times larger than the low-amplitude residuals. And this is exactly what is seen.

To investigate further the variations of the residual magnitudes seen in Fig. 9 a time-dependent analysis of the K2 photometry was performed, the results of which are graphically summarized in Figure 10. The data were subdivided into bins and for each bin Fourier first-term amplitudes $\left(A_{1}\right)$ and phases $\left(\phi_{1}\right)$ were calculated for the dominant and additional frequencies (eight harmonics for the dominant mode and three harmonics for the additional mode) and for the linear combination frequencies. The analysis was performed with our Fourier least-squares (LevenbergMarquardt) multi-frequency fitting program, taking as starting values the $P_{D}$ and $P_{A}$ values derived for the entire data set (see Table 3 ). Owing to the high precision (mmag), relatively short cadence $(30 \mathrm{~min})$, long baseline $(81.8 \mathrm{~d})$ and large number of K2 brightness measurements (N 3700), it was also possible to estimate directly the time variations of $P_{D}$ and $P_{A}$.

In Fig. 10 the upper three panels show the time variations for the dominant mode, and the lower three panels show the variations for the additional mode. In each panel two sets of points are plotted: (a) the solid dots were derived by dividing the photometry into eight non-overlapping 10day-wide bins and calculating the $A_{1}, \phi_{1}$ and $P$ values for each bin, the (labelled) horizontal line indicating the mean value of the eight bins. Each bin contained $\sim 480$ points, and the standard deviations of the residuals of the light curve fits ranged from 4.5 to 2.0 mmag. For both modes the mean $P$, $A$ and $\phi$ values agree well with the values derived from the combined $81.8 \mathrm{~d}$ data set. The $P_{D}$ value for each bin also was derived using the non-parametric phase dispersion method, specifically, PDM2 (Stellingwerf 1978, 2011), and the periods were found to be very similar to the values derived using our multi-frequency program; (b) the crosses were derived using seven overlapping 20-day-wide bins with 10-day offsets. Clearly both modes show amplitude, phase and period 

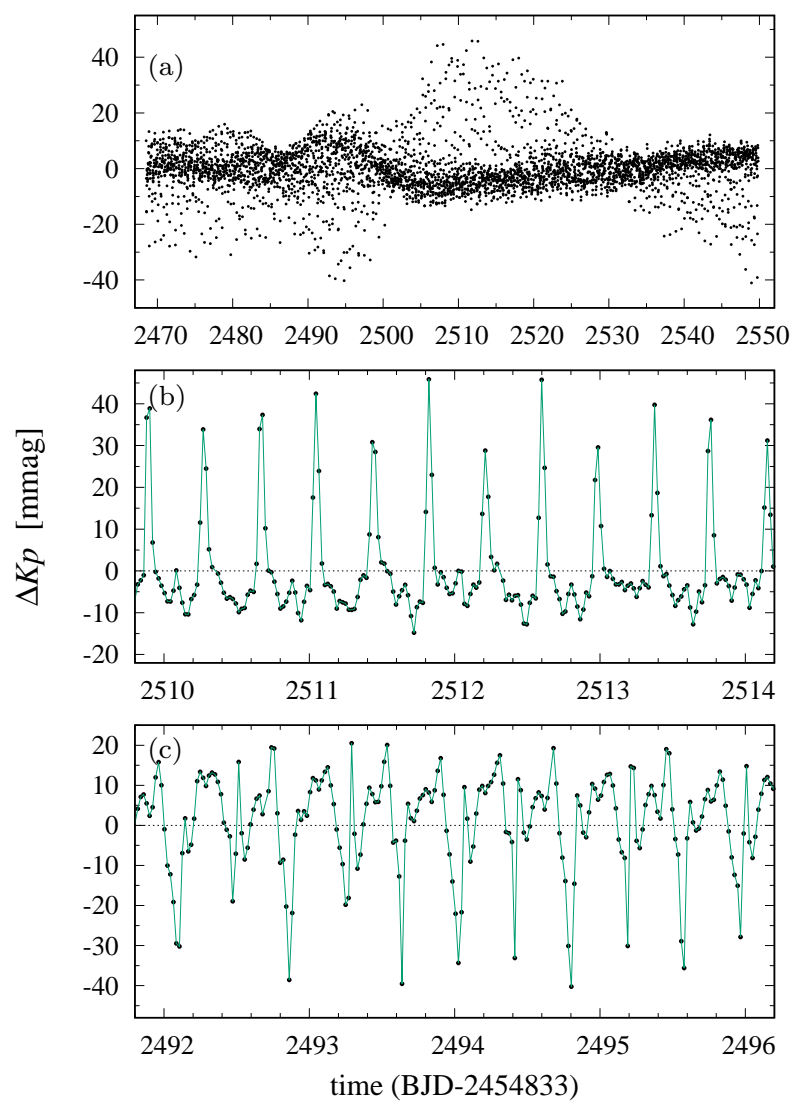

Figure 9. EPIC 216764000: residuals after prewhitening with $f_{D}=1 / 0.386748 \mathrm{~d}^{-1}, f_{A}=1 / 0.271468 \mathrm{~d}^{-1}$, their harmonics and linear combination frequencies. (a) Residuals over the entire 82.6d interval of the K2 data. (b) Close-up of a 4-d interval when the large-amplitude residuals are positive. (c) Close-up of a 4-d interval when the large-amplitude residuals are negative. The largeamplitude spikes are resolved (with 3-5 observations per spike) and have a period close to $P_{D}$.

variations, with the ranges of the variations exceeding the uncertainties of the individual measurements (as indicated by the error bars), indicating that the variations are almost certainly real and are the cause of the residuals shown in Fig. 9.

As expected the pulsation periods and phases are related for both modes. For the dominant mode the periods and phases are anticorrelated with the amplitudes, the smallest $A_{1, \mathrm{D}}$ values occuring shortly after the longest $P_{D}$ and greatest $\phi_{1, \mathrm{D}}$ values. For the additional mode all three quantities are correlated for the first 40 days, the smallest $A_{1, \mathrm{~A}}$ values occurring for the shortest $P_{A}$ periods, but then the correlation seems to break down for the last 20 days. An anticorrelation of the dominant period and amplitude was also seen for V1127 Aql (see fig. 12 of Chadid et al. 2010), a Blazhko star that we believe to be another pRRd star (see $\S 4.1$ below).

We cannot exclude that the observed variations of the pulsation modes in EPIC 216764000 might be periodic and that the star is undergoing a slow Blazhko modulation (like V1127 Aql). However, because the K2 time-base is only $82 \mathrm{~d}$, our data are insufficient to make such claims. What can be
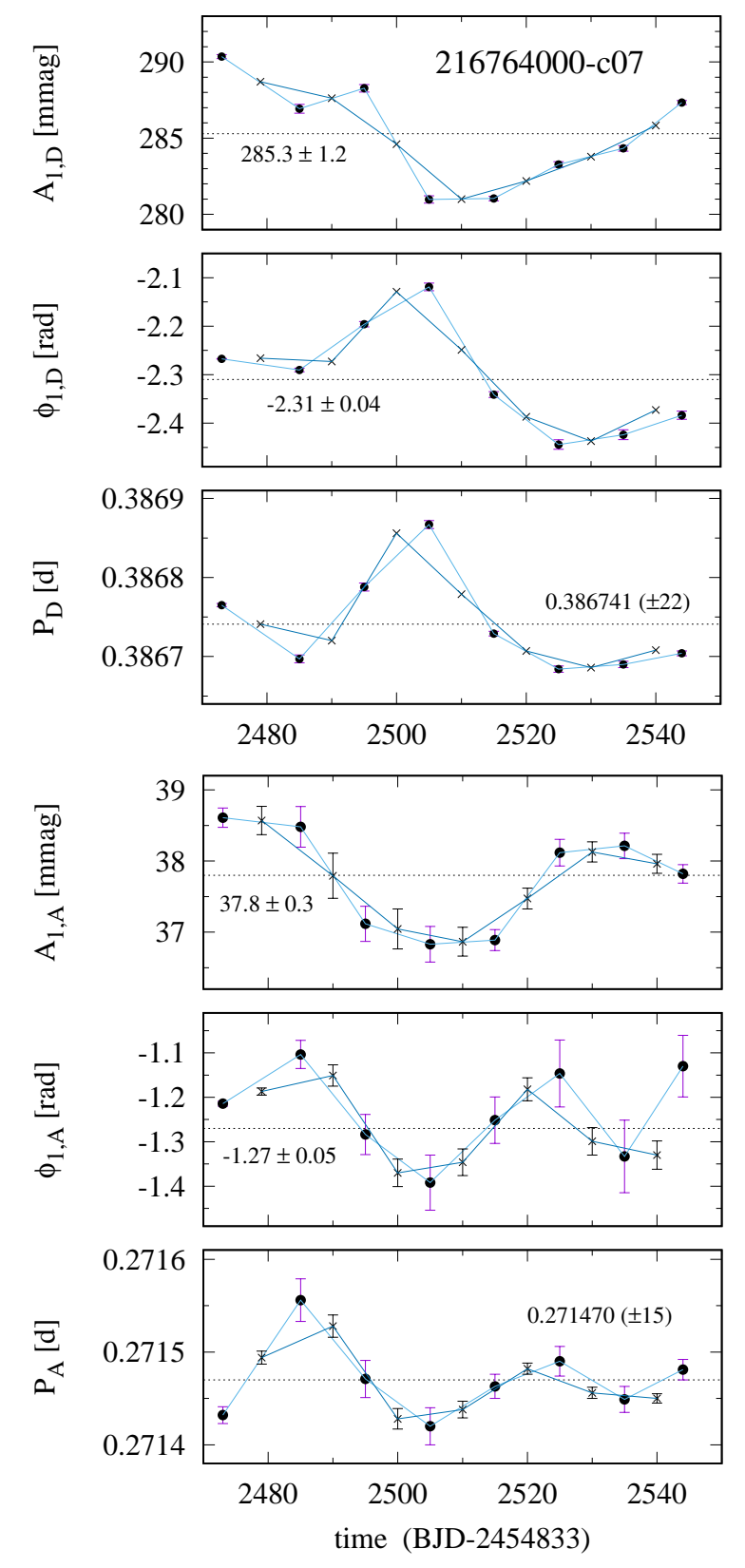

Figure 10. Time variations of the periods and Fourier first-term amplitudes and phases for the dominant (D) and additional (A) pulsation modes for EPIC 216764000. Clearly, both modes exhibit amplitude, phase and period variations.

concluded is that the data collected so far are consistent with non-repetitive (irregular) phase and amplitude variability.

\subsubsection{Long-term changes of the dominant period?}

The four main photometric data sets for EPIC 216764000 (Johannesburg plates measured by Uitterdijk, ASAS-3, SuperWASP and K2 - see Table 1) were acquired over more than 80 years, which usually is sufficient for deriving a period change rate, $\mathrm{d} P / \mathrm{d} t$. Unfortunately, the $\sim 65$-yr gap between the Johannesburg photographic plates (1928-1937) measured by Uitterdijk and the ASAS-3 (2001-2009) and 
SuperWASP (2006-2008) photometry makes keeping track of pulsation cycles almost impossible. When only the ASAS-3, SuperWASP and K2 observations are considered, the dominant period appears to be increasing, the estimated periods for the three epochs being $0.386708 \mathrm{~d}, 0.386718 \mathrm{~d}$ and $0.386748 \mathrm{~d}$, respectively. Adopting these periods, and their respective epochs of maximum light (see Table 1), and assuming a linearly increasing period gives an estimated $\mathrm{d} P / \mathrm{d} t=7.75 \times 10^{-9} \mathrm{~d} \mathrm{~d}^{-1}=2.83 \mathrm{~d} \mathrm{Myr}^{-1}$. Disappointingly, this period change rate gives a predicted period for the 1930 's that is considerably smaller than the period derived by Uitterdijk (which is mid-way between the ASAS-3 and SuperWASP values). One possible explanation for this high rate of period change, which is three orders of magnitude too fast to be of evolutionary origin, is that the period may have irregular variations such as were observed by Le Borgne et al. (2007) for many RRab stars (see their fig. 4). Or possibly the period change rate is non-linear. Almost certainly, the period is not constant.

\subsection{Campaign 11 Stars}

The three pRRd stars observed during Campaign 11 have mean $K p$ magnitudes ranging from 17.59 to 19.84 mag (see $\S 2.2$ ) and are considerably fainter than EPIC 216764000 . As noted above, all three stars were extensively observed by the OGLE-IV survey and are among the 42 'peculiar' RRd stars discussed by P17. Because the results of the frequency analyses of the K2 data are similar for the three stars they will be discussed as a group.

\subsubsection{Frequencies, Amplitudes and Phases}

Fourier amplitude spectra derived from the K2 photometry are plotted in Figure 11. The spectra are similar for all three stars and resemble closely that of EPIC 216764000. The highest peaks in the top panels of Fig. 11 reveal clearly the dominant frequencies, $f_{D}$, at $3.0692,3.5482$ and $2.7964 \mathrm{~d}^{-1}$, respectively. The Fourier first-term amplitudes of the dominant peak, $A_{1, \mathrm{D}}(K p)=284.3 \pm 1.5$, $237.7 \pm 2.6$ and $345.7 \pm 2.2 \mathrm{mmag}$ (with $\mathrm{S} / \mathrm{N}=77,61$ and $66)$, are comparable to the corresponding amplitude for EPIC 216764000 (=285.4 $\pm 0.2 \mathrm{mmag})$. Several of the observable harmonics of the dominant frequencies can be seen in the top panels and have been labelled; their presence is consistent with the stars having asymmetric light curves when phased with the dominant period, as shown in Fig. 8. Amplitude spectra after prewhitening with the dominant frequencies and their harmonics are plotted in the second row of Fig. 11. The highest peak in each panel (with $\mathrm{S} / \mathrm{N}=$ 31,17 and 20) reveals the presence of the respective $a d-$ ditional frequency, $f_{A}$, at $4.3222,5.0071$ and $4.0292 \mathrm{~d}^{-1}$. The amplitudes, $A_{1, \mathrm{~A}}(K p)=114.1 \pm 1.5,69.2 \pm 2.6$ and $97.4 \pm 2.2 \mathrm{mmag}$, are all higher than the corresponding amplitude for EPIC 216764000 (=37.8 $\pm 0.2 \mathrm{mmag})$. Also prominent are many peaks at combination frequencies involving $f_{A}$ and $f_{D}$, several of which have been labelled. The bottom two rows of Fig. 11 show close-ups in the vicinity of the $f_{D}$ and $f_{A}$ peaks. The average values of the measured FWHM widths of the dominant and additional peaks, $0.016 \mathrm{~d}^{-1}$ and $0.017 \mathrm{~d}^{-1}$, are only slightly larger than the reciprocal of
Table 5. EPIC 235839761 (= OGLE-BLG-RRLYR-16999): Fourier frequencies, amplitudes and phases derived from the K2 photometry (C112 PDCsap, range $47.7 \mathrm{~d}, \mathrm{~N}=2039$ ), assuming the time of maximum light from the OGLE-IV study (Soszyński et al. 2017b): $t_{0}=$ BJD $2454833.00000+2167.29828$ (=UT 2014 December 8, 19:09:31). The fitted mean magnitude was $\langle K p\rangle=$ $17.596 \pm 0.001 \mathrm{mag}$, with $\sigma$ (residuals $)=49 \mathrm{mmag}$.

\begin{tabular}{|c|c|c|c|c|c|}
\hline Label & $\begin{array}{l}\text { Frequency } \\
\qquad\left[\mathrm{d}^{-1}\right]\end{array}$ & $\begin{array}{l}\text { Period } \\
{[\mathrm{d}]}\end{array}$ & $\begin{array}{c}\text { Ampl. } \\
{\left[\begin{array}{c}\mathrm{mmag}] \\
\pm 1.5\end{array}\right.}\end{array}$ & $\begin{array}{l}\text { Phase } \\
{[\mathrm{rad}]}\end{array}$ & Sig \\
\hline \multicolumn{6}{|c|}{ (a) Dominant frequency, $f_{D}(=\mathrm{f} 1)$, and its harmonics } \\
\hline f1 & $\underline{3.06917}$ & 0.325821 & 284.3 & $-2.188 \pm 0.005$ & 452 \\
\hline $2 \mathrm{f} 1$ & $\overline{6.13834}$ & 0.162911 & 99.1 & $-1.546 \pm 0.016$ & 98 \\
\hline $3 \mathrm{f} 1$ & 9.20751 & 0.108607 & 39.1 & $-0.925 \pm 0.040$ & 89 \\
\hline $4 \mathrm{f} 1$ & 12.27668 & 0.081455 & 17.4 & $-0.240 \pm 0.088$ & 25 \\
\hline $5 \mathrm{f} 1$ & 15.34585 & 0.065164 & 5.7 & $0.532 \pm 0.272$ & 5 \\
\hline $6 \mathrm{f} 1$ & 18.41502 & 0.054303 & 3.5 & $0.880 \pm 0.444$ & $<5$ \\
\hline $7 \mathrm{f} 1$ & 21.48419 & 0.046546 & 1.9 & $2.070 \pm 0.826$ & $<5$ \\
\hline \multicolumn{6}{|c|}{ (b) Additional frequency, $f_{A}(=\mathrm{f} 2)$, and its harmonics } \\
\hline f2 & 4.32216 & 0.231366 & 114.1 & $2.036 \pm 0.014$ & 222 \\
\hline $2 \mathrm{f} 2$ & $\overline{8.64431}$ & 0.115683 & 13.2 & $0.512 \pm 0.117$ & 16 \\
\hline $3 \mathrm{f} 2$ & 12.96647 & 0.077122 & 1.3 & $-1.405 \pm 1.176$ & $<5$ \\
\hline \multicolumn{6}{|c|}{ (c) Linear combination frequencies } \\
\hline $\mathrm{f} 1+\mathrm{f} 2$ & 7.39133 & 0.135294 & 66.0 & $2.271 \pm 0.023$ & 189 \\
\hline $2 \mathrm{f} 1+\mathrm{f} 2$ & 10.46050 & 0.095598 & 35.0 & $2.831 \pm 0.044$ & 85 \\
\hline $3 \mathrm{f} 1+\mathrm{f} 2$ & 13.52967 & 0.073912 & 18.6 & $-2.636 \pm 0.082$ & 33 \\
\hline $4 \mathrm{f} 1+\mathrm{f} 2$ & 16.59884 & 0.060245 & 10.8 & $-2.013 \pm 0.143$ & 13 \\
\hline $5 \mathrm{f} 1+\mathrm{f} 2$ & 19.66801 & 0.050844 & 4.4 & $-1.135 \pm 0.347$ & $\sim 5$ \\
\hline $6 \mathrm{f} 1+\mathrm{f} 2$ & 22.73718 & 0.043981 & 1.7 & $-0.772 \pm 0.930$ & $<5$ \\
\hline $2 \mathrm{f} 1-\mathrm{f} 2$ & 1.81618 & 0.550605 & 7.2 & $2.292 \pm 0.214$ & 6 \\
\hline $3 \mathrm{f} 1-\mathrm{f} 2$ & 4.88535 & 0.204693 & 6.8 & $2.868 \pm 0.227$ & 7 \\
\hline $4 \mathrm{f} 1-\mathrm{f} 2$ & 7.95452 & 0.125715 & 5.6 & $-2.741 \pm 0.277$ & 5 \\
\hline $5 \mathrm{f} 1-\mathrm{f} 2$ & 11.02369 & 0.090714 & 2.7 & $-1.767 \pm 0.569$ & $<5$ \\
\hline $6 \mathrm{f} 1-\mathrm{f} 2$ & 14.09286 & 0.070958 & 2.0 & $-1.176 \pm 0.763$ & $<5$ \\
\hline $\mathrm{f} 2-\mathrm{f} 1$ & 1.25299 & 0.798093 & 21.4 & $-2.894 \pm 0.072$ & 47 \\
\hline $2 \mathrm{f} 2-\mathrm{f} 1$ & 5.57514 & 0.179368 & 3.8 & $1.052 \pm 0.406$ & $\sim 5$ \\
\hline $2 \mathrm{f} 2-2 \mathrm{f} 1$ & 2.50597 & 0.399047 & 6.7 & $3.033 \pm 0.230$ & $<5$ \\
\hline $\mathrm{f} 1+2 \mathrm{f} 2$ & 11.71348 & 0.085372 & 10.1 & $0.646 \pm 0.154$ & 11 \\
\hline $2 \mathrm{f} 1+2 \mathrm{f} 2$ & 14.78265 & 0.067647 & 8.7 & $1.053 \pm 0.177$ & 10 \\
\hline $3 \mathrm{f} 1+2 \mathrm{f} 2$ & 17.85182 & 0.056017 & 7.2 & $2.033 \pm 0.213$ & 6 \\
\hline $4 \mathrm{f} 1+2 \mathrm{f} 2$ & 20.92099 & 0.047799 & 3.1 & $2.775 \pm 0.490$ & $<5$ \\
\hline $5 \mathrm{f} 1+2 \mathrm{f} 2$ & 23.99016 & 0.041684 & 3.0 & $-2.693 \pm 0.504$ & $<5$ \\
\hline
\end{tabular}

the K2 Campaign 11 observation time interval, $1 / 74.2 \mathrm{~d}=$ $0.013 \mathrm{~d}^{-1}$.

Frequencies, amplitudes and phases derived from the K2 Campaign 11 data are summarized in Tables 5-7. The number of fitted frequencies for EPIC 235839761, 251248823 and $251248824(=29,23,33$ respectively) are based on the C112 data only and are a subset of all the fitted frequencies; they differ somewhat from the number of frequencies that were used by the FNPEAK analyses (C111+C112 datasets).

In Figure 12 amplitude spectra based on the OGLE-IV $I$-passband photometry for the three Campaign 11 stars are plotted. The top panels show peaks corresponding to the $f_{D}$ and $f_{A}$ frequencies, both of which are flanked by numerous one-day aliases. Prominent in the close-ups shown in the bottom two panels are the one-year alias frequencies at $\Delta f_{D} \pm 0.0027 \mathrm{~d}^{-1}$. As expected, given that the OGLE-IV data have a 7.5-year time-base compared with only 74.2 days for the $K 2$ data, the $f_{D}$ and $f_{A}$ peaks are much narrower (FWHM widths $\sim 0.0004 \mathrm{~d}^{-1}$ ) than those seen in Fig. 11 for the $K 2$ data.

The modulation of EPIC 235839761 is revealed in the bottom left panel of Fig. 12 by the significant extra sidepeak near $4.32305 \mathrm{~d}^{-1}$, which corresponds to a modulation period $538 \mathrm{~d}$. On the other hand, the modulation of the dominant mode of EPIC 251248824 is not confirmed (see bottom right panels of Fig. 12). It appears that there is modulation but that it is of the additional mode, with the same period and amplitude as claimed for the 

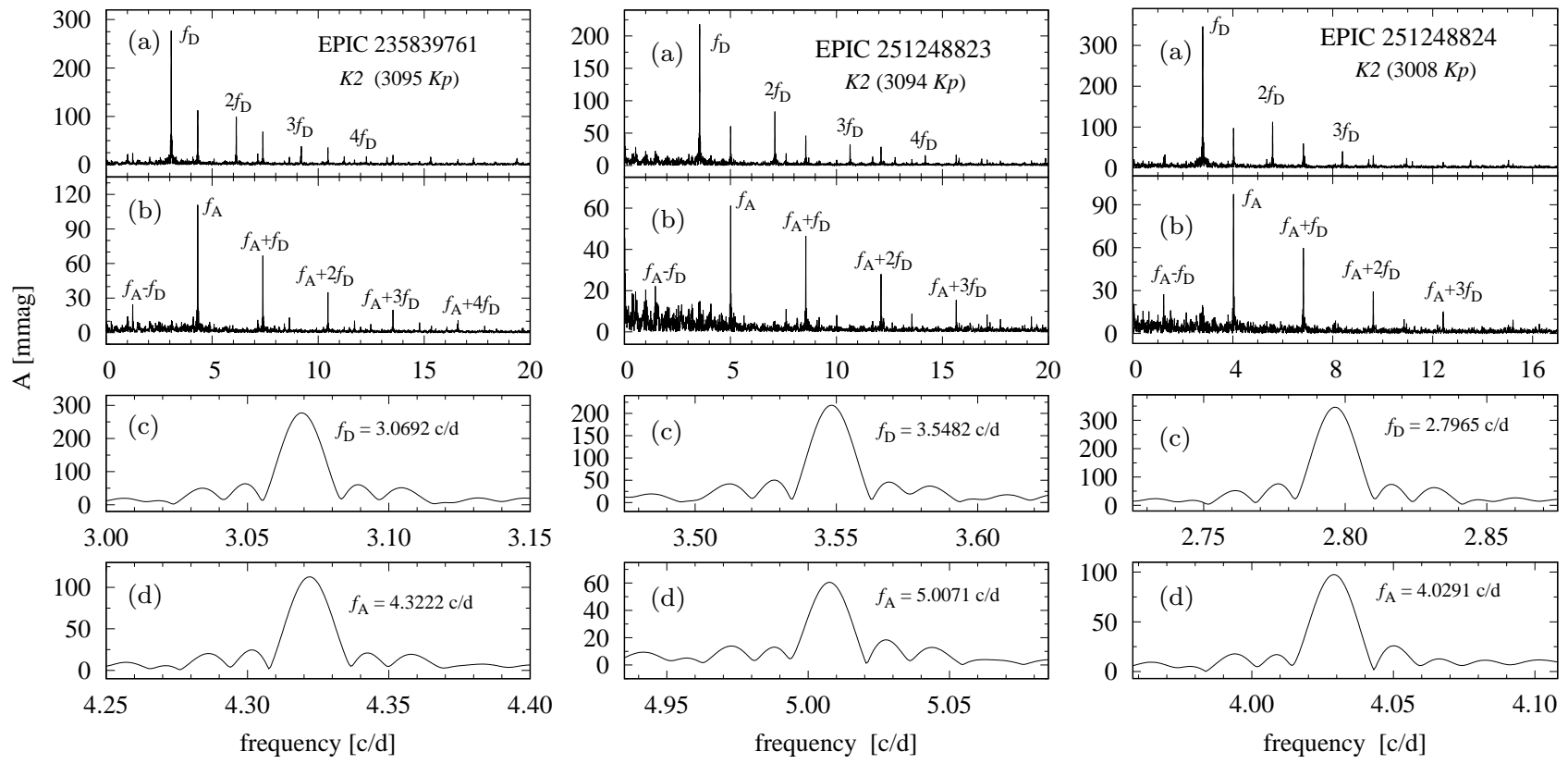

Figure 11. Fourier amplitude spectra for the three Campaign 11 pRRd stars, where the spectra for each star were derived using more than 3000 PDCsap measurements acquired by K2 over 74 days in 2016 . The top two panels for each star show: (a) the dominant peaks at frequency $f_{D}$ and their harmonics (a few of which are labelled); and (b) after prewhitening with $f_{D}$ and its harmonics, the additional frequency peak at $f_{A}$ and prominent peaks corresponding to linear combinations of $f_{A}$ and $f_{D}$ (several of which are labelled) - the harmonics at $2 f_{A}$ are barely visible. See Fig.6g for window function. The bottom panels (c, d) show close-ups of the $f_{D}$ and $f_{A}$ peaks.

EPIC 235839761

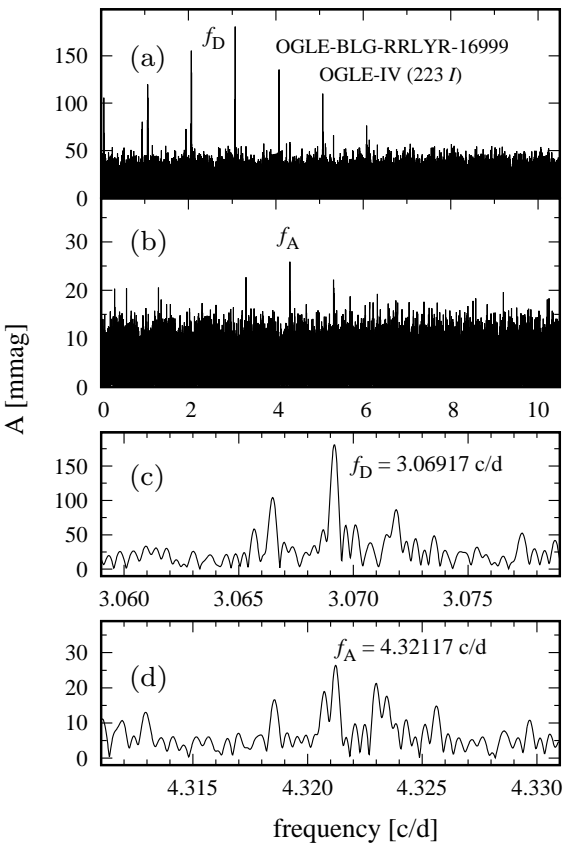

EPIC 251248823
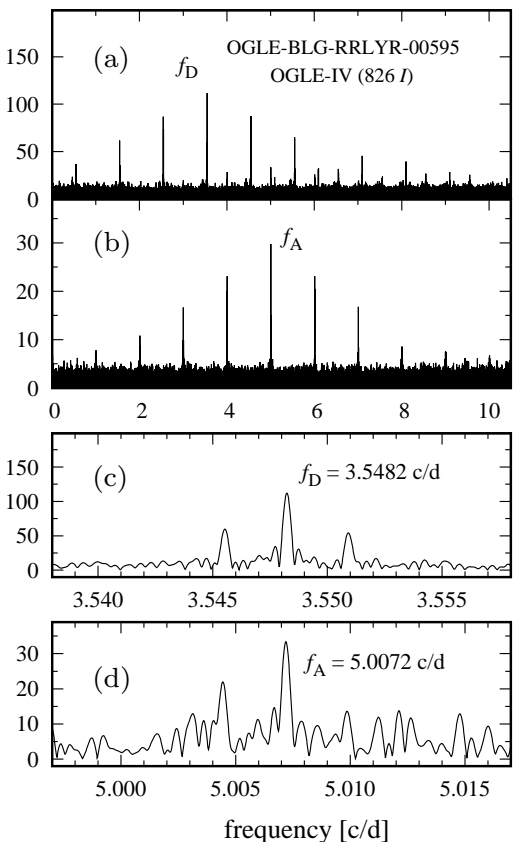

EPIC 251248824
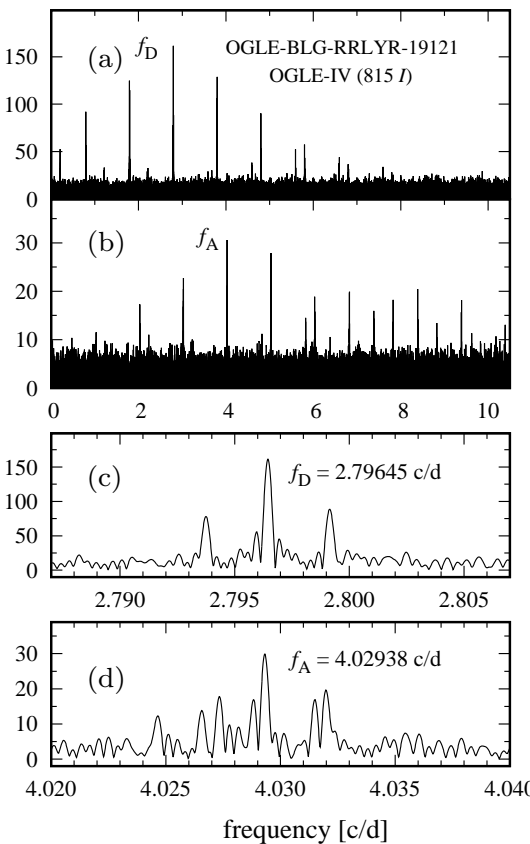

Figure 12. Fourier amplitude spectra derived from ground-based OGLE-IV $I$-band photometry (acquired over 7.3 years, from 2010 to 2017). The upper panels for each star reveal the 'dominant' and 'additional' frequencies, where the extra peaks are mainly one-day aliasing peaks. The lower panels show close-ups in the vicinity of $f_{D}$ and $f_{A}$, prominently showing one-year aliasing peaks at $\Delta \mathrm{f}=$ $\pm 0.0027 \mathrm{~d}^{-1}$. The double peaks in the bottom panel for EPIC 235839761 (left) reveal the $507 \pm 11 \mathrm{~d}$ modulation of the additional mode, while for EPIC 251248824 (right) there is no evidence for the $482 \pm 5 \mathrm{~d}$ modulation of the dominant mode claimed by P17; instead one sees evidence for a $481 \mathrm{~d}$ modulation of the additional mode. 
Table 6. EPIC 251248823 (= OGLE-BLG-RRLYR-00595): Fourier frequencies, amplitudes and phases derived from the K2 photometry (C112 PDCsap with additional detrending and normalization to $\langle K p\rangle=18.826 \pm 0.002 \mathrm{mag}, \mathrm{N}=2039, \sigma$ (residuals $)=90 \mathrm{mmag}$ ). For the phases we adopted $t_{0}=$ BJD $2454833.00000+2167.05823$ (=UT 2014 December 8, 13:23:51), the value from the latest OGLE-IV study (Soszyński et al. 2017b).

\begin{tabular}{|c|c|c|c|c|c|}
\hline Label & $\begin{array}{c}\text { Frequency } \\
{\left[\mathrm{d}^{-1}\right]}\end{array}$ & $\begin{array}{l}\text { Period } \\
\text { [d] }\end{array}$ & $\begin{array}{c}\text { Ampl. } \\
{[\mathrm{mmag}]} \\
\pm 2.6\end{array}$ & $\begin{array}{l}\text { Phase } \\
{[\mathrm{rad}]}\end{array}$ & Sig \\
\hline \multicolumn{6}{|c|}{ (a) Dominant frequency, $f_{D}(=\mathrm{f} 1)$, and its harmonics } \\
\hline f1 & 3.54822 & 0.281831 & 237.7 & $-2.064 \pm 0.011$ & 324 \\
\hline $2 \mathrm{f} 1$ & 7.09645 & 0.140916 & 87.9 & $-1.563 \pm 0.030$ & 110 \\
\hline $3 \mathrm{f} 1$ & 10.64467 & 0.093944 & 40.1 & $-0.989 \pm 0.065$ & 43 \\
\hline $4 \mathrm{f} 1$ & 14.19290 & 0.070458 & 19.8 & $-0.231 \pm 0.132$ & 13 \\
\hline $5 \mathrm{f} 1$ & 17.74112 & 0.056366 & 7.7 & $0.177 \pm 0.339$ & 4 \\
\hline $6 \mathrm{f} 1$ & 21.28935 & 0.046972 & 5.6 & $0.807 \pm 0.468$ & $<4$ \\
\hline \multicolumn{6}{|c|}{ (b) Additional frequency, $f_{A}(=\mathrm{f} 2)$, and its harmonics } \\
\hline $\mathrm{f} 2$ & 5.00709 & 0.199717 & 69.2 & $-1.211 \pm 0.038$ & 98 \\
\hline $2 f 2$ & 10.01417 & 0.099859 & 10.7 & $0.632 \pm 0.245$ & 5 \\
\hline \multicolumn{6}{|c|}{ (c) Linear combination frequencies } \\
\hline $\mathrm{f} 1+\mathrm{f} 2$ & 8.55531 & 0.116886 & 49.0 & $-0.698 \pm 0.053$ & 55 \\
\hline $2 \mathrm{f} 1+\mathrm{f} 2$ & 12.10353 & 0.082621 & 32.3 & $-0.238 \pm 0.080$ & 29 \\
\hline $3 \mathrm{f} 1+\mathrm{f} 2$ & 15.65176 & 0.063891 & 16.3 & $0.550 \pm 0.160$ & 10 \\
\hline $4 \mathrm{f} 1+\mathrm{f} 2$ & 19.19998 & 0.052083 & 11.3 & $0.987 \pm 0.231$ & 7 \\
\hline $5 \mathrm{f} 1+\mathrm{f} 2$ & 22.74821 & 0.043960 & 7.7 & $2.066 \pm 0.333$ & $<5$ \\
\hline $6 \mathrm{f} 1+\mathrm{f} 2$ & 26.29643 & 0.038028 & 2.1 & $3.022 \pm 1.276$ & $<5$ \\
\hline $\mathrm{f} 2-\mathrm{f} 1$ & 1.45886 & 0.685467 & 26.2 & $-0.593 \pm 0.100$ & 24 \\
\hline $2 \mathrm{f} 1-\mathrm{f} 2$ & 2.08936 & 0.478615 & 5.6 & $-0.881 \pm 0.462$ & $<5$ \\
\hline $3 \mathrm{f} 1-\mathrm{f} 2$ & 5.63759 & 0.177381 & 11.8 & $-0.525 \pm 0.222$ & 7 \\
\hline $4 \mathrm{f} 1-\mathrm{f} 2$ & 9.18581 & 0.108863 & 8.4 & $1.021 \pm 0.313$ & 6 \\
\hline $6 \mathrm{f} 1-\mathrm{f} 2$ & 16.28226 & 0.061417 & 3.8 & $2.043 \pm 0.690$ & $<5$ \\
\hline $\mathrm{f} 1+2 \mathrm{f} 2$ & 13.56239 & 0.073733 & 9.3 & $0.841 \pm 0.278$ & 7 \\
\hline $2 \mathrm{f} 1+2 \mathrm{f} 2$ & 17.11062 & 0.058443 & 7.2 & $1.886 \pm 0.360$ & 7 \\
\hline $3 \mathrm{f} 1+2 \mathrm{f} 2$ & 20.65884 & 0.048405 & 5.8 & $2.119 \pm 0.449$ & $<5$ \\
\hline $4 \mathrm{f} 1+2 \mathrm{f} 2$ & 24.20707 & 0.041310 & 5.2 & $2.712 \pm 0.501$ & $<5$ \\
\hline
\end{tabular}

dominant mode. Panel (c) shows the 'dominant' peak at $f_{D}=2.79645 \mathrm{~d}^{-1} \quad\left(A_{D}=157.7 \mathrm{mmag}\right)$ and its one-year alias peaks at $f_{D} \pm 0.00274 \mathrm{~d}^{-1}$. Panel (d) shows the 'additional' peak at $f_{A}=4.02938 \mathrm{~d}^{-1}$ (amplitude $30 \mathrm{mmag}$ ), its one-year alias peaks (again offset by $\pm 0.00274 \mathrm{~d}^{-1}$ ), and extra interior side-peaks at $f_{A} \pm 0.00208 \mathrm{~d}^{-1}$ (amplitude $18 \mathrm{mmag}$ ), the difference corresponding to $P_{B}=481 \mathrm{~d}$.

\subsubsection{Light Curves and Fourier parameters}

Light curves derived from the $K 2$ photometry are plotted in Figure 13. The three panels for each star are as described for EPIC 216764000 (see $§ 3.1 .2$ and Fig. 7). The top row shows light curves phased with the OGLE-IV dominant period and times of maximum light. Use of the latest high-precision OGLE-IV periods and $t_{0}$-values served as a useful check on the $K 2$ periods, and showed that the light curves constructed with the K2 photometry are not offset in time or phase from the OGLE-IV light curves. For all three stars the influence of the combination frequencies on the overall light curve is seen to be comparable to that of the additional mode.

Fourier decomposition parameters, total amplitudes, and risetimes for the Campaign 11 pRRd stars, all derived from the $K p$ photometry, are given in columns (3)-(5) of Table 4. For comparison purposes OGLE-IV total amplitudes and Fourier parameters derived from the $I$-passband data, and the differences, $\Delta$, between the $K 2 K p$ and OGLE-IV $I$ values, are also recorded in the Table 4 . The dominant-mode total amplitudes are similar for all four stars, but the additional-mode total amplitude is smallest for EPIC 216764000. As expected the $K p$ total amplitudes are larger than the $I$-passband total amplitudes, and the
Table 7. EPIC 251248824 (= OGLE-BLG-RRLYR-19121): significant frequencies derived from $K 2$ photometry $(\mathrm{C} 111+\mathrm{C} 112$, PDCsap, mean magnitude $\langle K p\rangle=19.844 \pm 0.002 \mathrm{mag}, \mathrm{N}=3087$, $\sigma($ residuals $)=85 \mathrm{mmag})$. For the phases the assumed time of maximum light was $t_{0}=$ BJD $2454833.00000+2167.13778$ (=UT 2014 December 8, 15:18:24), the value from the latest OGLE-IV study.

\begin{tabular}{|c|c|c|c|c|c|}
\hline Label & $\begin{array}{c}\text { Frequency } \\
{\left[\mathrm{d}^{-1}\right]}\end{array}$ & $\begin{array}{l}\text { Period } \\
\text { [d] }\end{array}$ & $\begin{array}{c}\text { Ampl. } \\
{[\mathrm{mmag}]} \\
\pm 2.2\end{array}$ & $\begin{array}{l}\text { Phase } \\
\text { [rad] }\end{array}$ & Sig \\
\hline \multicolumn{6}{|c|}{ (a) Dominant frequency, $f_{D}(=\mathrm{f} 1)$, and its harmonics } \\
\hline f1 & 2.79644 & 0.357597 & 345.7 & $-2.250 \pm 0.006$ & 491 \\
\hline $2 \mathrm{f} 1$ & 5.59289 & 0.178798 & 112.0 & $-1.459 \pm 0.019$ & 190 \\
\hline $3 \mathrm{f} 1$ & 8.38933 & 0.119199 & 41.9 & $-0.820 \pm 0.051$ & 63 \\
\hline $4 \mathrm{f} 1$ & 11.18578 & 0.089399 & 17.3 & $-0.081 \pm 0.125$ & 14 \\
\hline $5 \mathrm{f} 1$ & 13.98222 & 0.071519 & 4.7 & $0.893 \pm 0.458$ & $<5$ \\
\hline \multicolumn{6}{|c|}{ (b) Additional frequency, $f_{A}(=\mathrm{f} 2)$, and its harmonics } \\
\hline f2 & 4.02916 & 0.248191 & 97.4 & $-1.756 \pm 0.022$ & 190 \\
\hline $2 \mathrm{f} 2$ & $\overline{8.05831}$ & 0.124096 & 6.7 & $-1.234 \pm 0.323$ & $<5$ \\
\hline $3 \mathrm{f} 2$ & 12.08746 & 0.082730 & 2.6 & $2.256 \pm 0.836$ & $<5$ \\
\hline $4 \mathrm{f} 2$ & 16.11662 & 0.062048 & 3.5 & $-0.357 \pm 0.611$ & $<5$ \\
\hline \multicolumn{6}{|c|}{ (c) Linear combination frequencies } \\
\hline $\mathrm{f} 1+\mathrm{f} 2$ & 6.82560 & 0.146507 & 59.0 & $-1.276 \pm 0.037$ & 108 \\
\hline $2 \mathrm{f} 1+\mathrm{f} 2$ & 9.62204 & 0.103928 & 29.3 & $-0.703 \pm 0.074$ & 35 \\
\hline $3 \mathrm{f} 1+\mathrm{f} 2$ & 12.41849 & 0.080525 & 14.6 & $-0.106 \pm 0.147$ & 11 \\
\hline $4 \mathrm{f} 1+\mathrm{f} 2$ & 15.21493 & 0.065725 & 9.0 & $0.596 \pm 0.240$ & 5 \\
\hline $5 \mathrm{f} 1+\mathrm{f} 2$ & 18.01138 & 0.055521 & 3.4 & $2.151 \pm 0.634$ & $<5$ \\
\hline $2 \mathrm{f} 1-\mathrm{f} 2$ & 1.56373 & 0.639495 & 10.0 & $-0.469 \pm 0.215$ & 7 \\
\hline $3 \mathrm{f} 1-\mathrm{f} 2$ & 4.36018 & 0.229348 & 3.5 & $1.231 \pm 0.616$ & $<5$ \\
\hline $4 \mathrm{f} 1-\mathrm{f} 2$ & 7.15662 & 0.139731 & 3.0 & $1.273 \pm 0.722$ & $<5$ \\
\hline $5 \mathrm{f} 1-\mathrm{f} 2$ & 9.95307 & 0.100472 & 3.7 & $1.132 \pm 0.578$ & $<5$ \\
\hline $\mathrm{f} 2-\mathrm{f} 1$ & 1.23271 & 0.811221 & 27.0 & $0.128 \pm 0.080$ & 31 \\
\hline $2 \mathrm{f} 2-\mathrm{f} 1$ & 5.26187 & 0.190047 & 5.8 & $2.731 \pm 0.372$ & $<5$ \\
\hline $\mathrm{f} 1+2 \mathrm{f} 2$ & 10.85475 & 0.092126 & 9.7 & $-0.384 \pm 0.223$ & 5 \\
\hline $2 \mathrm{f} 1+2 \mathrm{f} 2$ & 13.65120 & 0.073254 & 3.9 & $0.713 \pm 0.555$ & $<5$ \\
\hline $3 \mathrm{f} 1+2 \mathrm{f} 2$ & 16.44764 & 0.060799 & 2.9 & $1.559 \pm 0.746$ & $<5$ \\
\hline $4 \mathrm{f} 2-5 \mathrm{f} 1$ & 2.13440 & 0.468516 & 13.4 & $-0.008 \pm 0.161$ & 10 \\
\hline $3 \mathrm{f} 1-2 \mathrm{f} 2$ & 0.33102 & 3.020930 & 9.0 & $0.053 \pm 0.241$ & $<5$ \\
\hline $3 \mathrm{f} 2-4 \mathrm{f} 1$ & 0.90169 & 1.109033 & 7.9 & $-1.477 \pm 0.272$ & 5 \\
\hline $2 \mathrm{f} 2-2 \mathrm{f} 1$ & 2.46542 & 0.405610 & 6.0 & $1.827 \pm 0.357$ & $<5$ \\
\hline $6 \mathrm{f} 1-4 \mathrm{f} 2$ & 0.66205 & 1.510465 & 5.3 & $-1.990 \pm 0.410$ & $<5$ \\
\hline $3 \mathrm{f} 2-3 \mathrm{f} 1$ & 3.69813 & 0.270407 & 5.0 & $-2.803 \pm 0.435$ & $<5$ \\
\hline $5 \mathrm{f} 1-2 \mathrm{f} 2$ & 5.92391 & 0.168807 & 4.5 & $-0.596 \pm 0.480$ & $<5$ \\
\hline $7 \mathrm{f} 1-4 \mathrm{f} 2$ & 3.45849 & 0.289143 & 3.5 & $-0.549 \pm 0.622$ & $<5$ \\
\hline $4 \mathrm{f} 1-2 \mathrm{f} 2$ & 3.12747 & 0.319747 & 3.4 & $-0.341 \pm 0.641$ & $<5$ \\
\hline $5 \mathrm{f} 1-3 \mathrm{f} 2$ & 1.89476 & 0.527772 & 3.1 & $0.569 \pm 0.704$ & $<5$ \\
\hline
\end{tabular}

mean differences between the K2 and OGLE-IV $R_{21}$ and $R_{31}$ ratios are practically negligible, $0.03 \pm 0.01$ and $0.02 \pm 0.01$. The mean differences between the phase parameters are also small, $0.16 \pm 0.11$ for $\Delta \phi_{21}$ and $0.21 \pm 0.11$ for $\Delta \phi_{31}$.

Table 4 also reveals that the Fourier parameters and other descriptors for all four stars are quite similar. Inspection of the amplitude ratios $\left(R_{21}, R_{31}, R_{41}, \ldots\right)$ reveals an exponential fall-off, at least for the first four terms. A similar fall-off was seen for the harmonic components of the RR Lyrae star V1127 Aql observed by CoRoT (see Chadid et al. 2010, and $\S 4.1$ below). One also sees close agreement between the parameter values derived from the $K p$ and OGLE $I$-photometry.

\subsubsection{Amplitude and Phase modulations}

As noted previously P17 found, based on OGLE I photometry, that EPIC 235839761 and EPIC 251248824 have modulated amplitudes (see last paragraph of $\S 2.3 .2$, and $\S 3.2 .1$ ). Unfortunately, the $74.2 \mathrm{~d}$ observation interval of the K2 Campaign 11 observations is insufficient to confirm such longperiod modulations, but the data do permit the investigation of possible shorter-time-scale variations in the same way as described above for EPIC 216764000 (see Fig. 10), i.e., by binning the $K 2$ data and analyzing the photometry in 

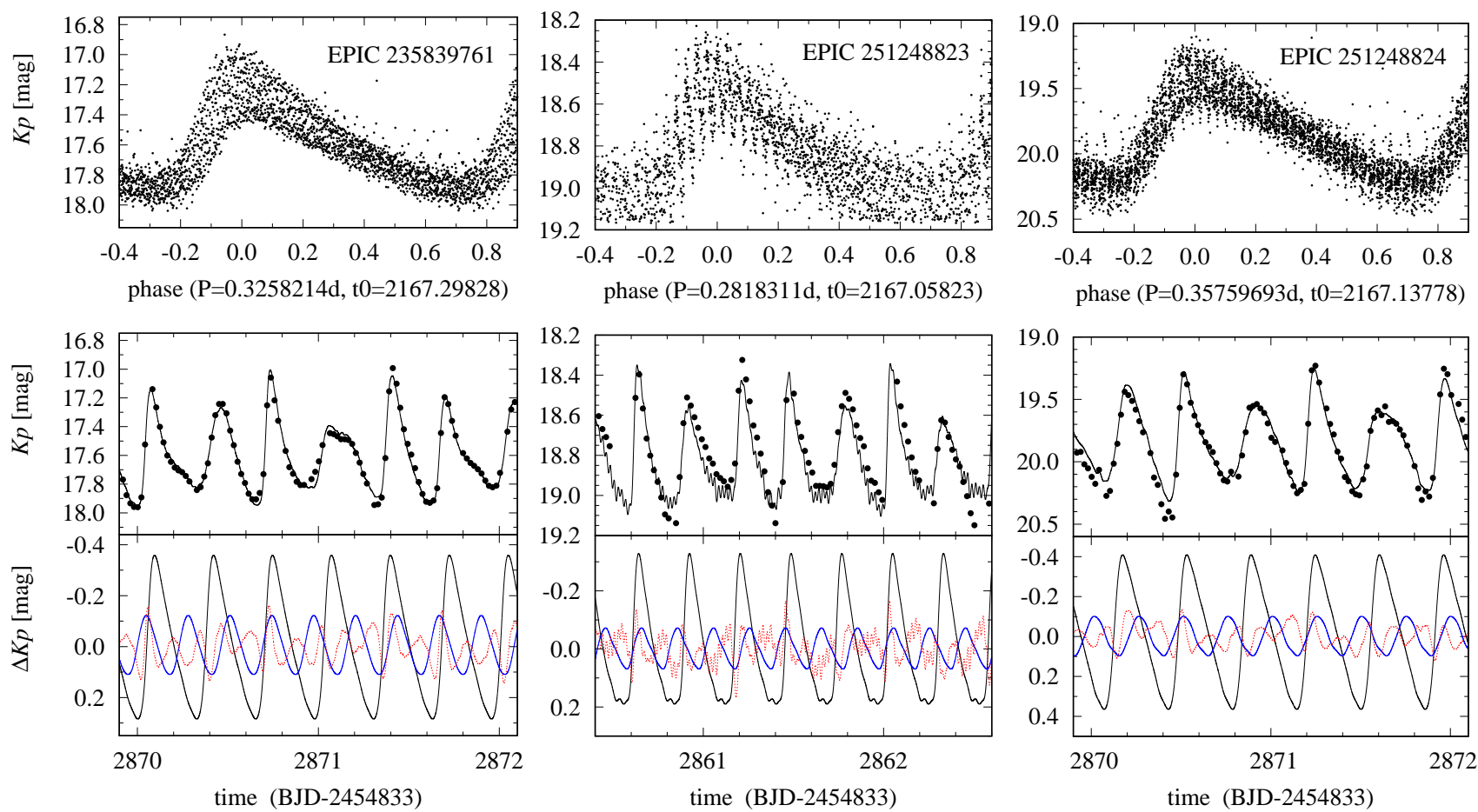

Figure 13. Light curves for the three pRRd stars observed during Campaign 11. (Top row) Photometry phased with the OGLE-IV dominant periods and times of maximum light; for EPIC 251248823 only the C112 data are plotted. (Middle row) Observed and fitted light curves ( $2.2 \mathrm{~d}$ segments). (Bottom row) Component light curves derived from the fitted two-frequency model. As was the case for EPIC 216764000 (see Fig. 7) the amplitudes of the combination frequencies (dotted red curves) are comparable in strength to those of the additional modes (blue curves).
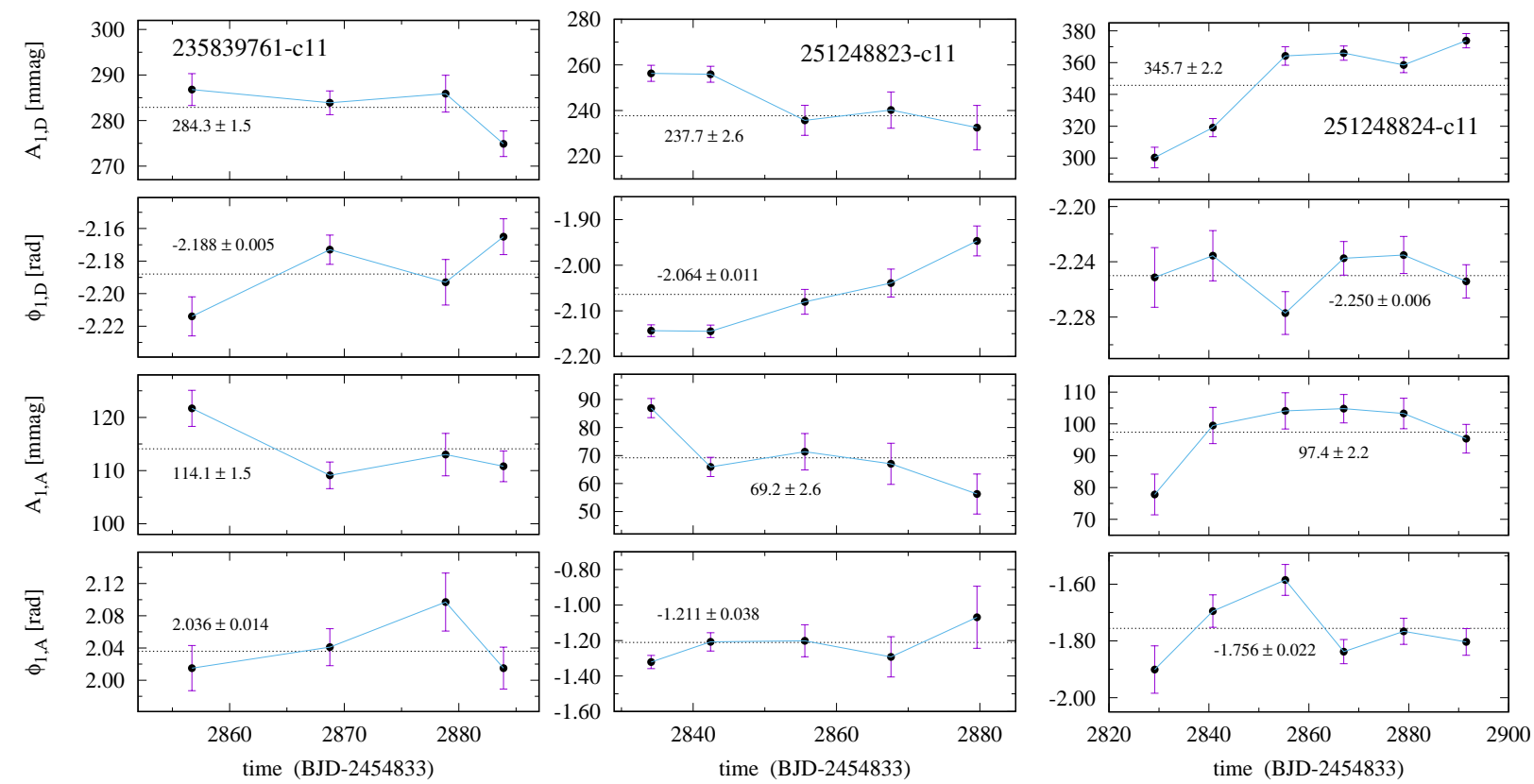

Figure 14. Time variations for the three Campaign $11 \mathrm{pRRd}$ stars, assuming the same periods and times of maximum light used for Tables 5-7. For each star the top two panels show the variations of the Fourier amplitudes and phases of the dominant mode, and the bottom two panels show the variations for the additional mode. Horizontal lines are drawn at the average values (see labels). 
each bin separately. The resulting time-variation graphs are shown in Figure 14. Several possible trends can be seen; however, because the Campaign 11 data were divided into two subcampaigns having different detector sensitivities we could not be confident that the trends are real. Nevertheless, and despite the three stars being significantly fainter than EPIC 216764000, we are encouraged that there is considerable internal consistency of the amplitudes and phases.

\subsubsection{Reanalysis of the OGLE photometry}

The photometry analyzed by P17 included all the available OGLE observations made up to and including the 2013 observing season. Since then the OGLE-IV team has acquired four more years of photometry. Primarily because of the extra observations, but also as a check on the previously derived periods, amplitudes and their ratios, the photometric observations made through 2017 for the 42 known pRRd stars were analyzed using our non-linear least-squares multi-frequency fitting program. The fits to the data were conducted with six harmonics for the dominant mode and three harmonics for the additional mode (including linear combination frequencies), usually with no adjustments for trends but when necessary with additional trend-fitting. For those stars with OGLE-II, -III and -IV I-band photometry the data sets were merged. For several such stars discontinuities in the photometric time series were found, in which case only the OGLE-IV data were analyzed.

The resultant mean $I$-magnitudes, periods, first-term Fourier amplitudes and their ratios are given in Table 8. In general there is agreement with the values given in Table 1 of $\mathrm{P} 17$, and with the more recent values given in the OGLE-IV on-line catalogue (http://ogledb.astrouw.edu.pl/ogle/OCVS/). Ten of the 42 stars were found by P17 to exhibit Blazhko modulations of either the dominant mode, the additional mode, or both modes (the ten stars are identified with an asterisk following the star number in the first column of Table 8). Except for the three stars with K2 and OGLE photometry (see §3.2.3) no attempt was made to identify additional Blazhko modulations over and above those identified by P17. In the discussion that follows the newly derived periods and amplitudes, as well as additional Fourier decomposition parameters, are used for the comparison of pRRd stars with classical (cRRd) and anomalous RRd (aRRd) stars.

\section{DISCUSSION}

\subsection{Pulsation Periods}

In the Petersen diagram (Fig. 1) the 'peculiar' RRd stars lie very roughly along the extension of the curve defined by the 'classical' RRd stars. While cRRd stars form a well-defined narrow sequence in the diagram, the pRRd and aRRd stars form large clouds with no apparent structure. The period ratios, $P_{S} / P_{L}$, for the $\mathrm{pRRd}$ stars vary from 0.682 to 0.720 , the range of which, 0.038 , is almost twice as big as the range seen for cRRd stars, 0.022 (from 0.726 to 0.748 ), and all but four of the shortest-period pRRd stars lie below the extrapolated cRRd sequence. While the fundamental-mode periods of the shortest-period cRRd stars extend down to $P_{L}=0.35 \mathrm{~d}$, the longest-period pRRd star has $P_{L}=0.41 \mathrm{~d}$; thus, in the period range $0.35<P_{L}<0.41 \mathrm{~d}$ the two groups coexist. The $17 \mathrm{pRRd}$ stars in this range amount to 40 per cent of the P17 sample and all have significantly smaller period ratios than do the stars on the cRRd sequence.

Assuming that the pRRd variable stars are single stars, pulsationally unstable in the fundamental and first-overtone radial modes as they evolve through the Instability Strip, and that they have physical characteristics within the ranges seen for 'normal' $R R$ Lyrae stars, i.e., $M \in(0.50-0.70) \mathrm{M}_{\odot}$, $\mathrm{L} \in(40-70) \mathrm{L}_{\odot}, T_{\text {eff }} \in(5000-8000) \mathrm{K}$, and $[\mathrm{Fe} / \mathrm{H}]$ in the range -2.5 dex (very metal poor) to 0.0 dex (solar), P17 were unable, using the linear nonadiabatic pulsation models of Smolec et al. (2016), to reproduce the periods and period ratios as low as those observed. The closest match was with the most extreme model, that with the highest mass $\mathrm{M}=0.70 \mathrm{M}_{\odot}$, lowest luminosity $\mathrm{L}=40 \mathrm{~L} \odot$, highest metallicity $[\mathrm{Fe} / \mathrm{H}]=0.0 \mathrm{dex}$, and hottest effective temperature. However, for the $T_{\text {eff }}$ range where both modes are unstable none of the models matched the observed periods and period ratios. As a result, P17 rejected the conclusion that the additional lowamplitude mode detected in the pRRd stars can be identified with the first-overtone radial pulsation, i.e., that the $\mathrm{pRRd}$ stars are simply very short-period cRRd stars.

A similar grid of linear models was calculated by Chadid et al. (2010). Their models, which are graphically summarized in their fig. 8 , assumed $\mathrm{L}$ in the range $40-70 \mathrm{~L}_{\odot}, \mathrm{M}$ in the range $0.50-0.80 \mathrm{M}_{\odot}$, and $T_{\text {eff }}$ in the range $5000-8000 \mathrm{~K}$, with metallicities ranging from metal poor, $[\mathrm{Fe} / \mathrm{H}]=-2.3 \mathrm{dex}$ (i.e., $\mathrm{Z}=0.0001$ ), to solar, 0.0 dex (i.e., $\mathrm{Z}=0.0200$ ). The models were computed for fundamental-mode periods as short as $0.22 \mathrm{~d}$, i.e., for the entire range of the observed longer-periods of the pRRd stars. The most metal-rich of the models predicted period ratios that are similar to those of the pRRd stars, but the match could be achieved only for the highest period ratios observed, 0.71-0.72. If models more metalrich than solar had been calculated by Chadid et al. the locations in the Petersen diagram of the solutions probably would have extended further into the pRRd domain. The possibility that pRRd stars are more metal-rich than the Sun needs to be tested with spectra, EPIC 216764000 with $<K p>=13.74$ mag being a good candidate for such spectroscopy.

Comparing the models of Chadid et al. and the models of Smolec et al. we see that they agree remarkably well. For $[\mathrm{Fe} / \mathrm{H}]=0.0$ dex (red dots in fig. 10 of P17 and black dots in fig. 8 of Chadid et al.) the period ratios, $P_{S} / P_{L}$, are almost the same in both computations. An excellent agreement of the computed period ratios is also seen for $[\mathrm{Fe} / \mathrm{H}]=-1.0$ dex. Both sets of models show that the period ratio has very little sensitivity to mass or luminosity and depends mostly on $[\mathrm{Fe} / \mathrm{H}]$. Where there is a difference is that $\mathrm{P} 17$ considered in their analysis also the growth rates of the modes. This information was used as an additional constraint, requiring that both radial modes have to be linearly unstable, thus limiting the period range of the acceptable models. It is for this reason that the P17 models do not extend to very short periods. For such short-period models at least one of the radial modes becomes damped and so the models must be rejected. Chadid et al. did not use the information about stability of the modes and no such additional constraint was imposed in their fig. 8. 
Table 8. Analysis of the OGLE $I$-photometry (2001-2017) for the 42 pRRd stars listed in Table 1 of Prudil et al. (2017), sorted according to decreasing amplitude ratio, $A_{1, D} / A_{1, A}$. The analyses were made using our non-linear least-squares (Levenberg-Marquardt) multi-frequency program, which, in addition to solving for the dominant and additional amplitudes and phases, also solved for the two independent frequencies (with error estimates). The identifications in the first column are the OGLE-BLG-RRLYR star numbers, underlined for the three stars observed by $K 2$, and followed by an asterisk if either $P_{D}$, $P_{A}$ or both exhibits Blazhko modulations (see Table 2 of P17). Column (2) contains the epochs of the analyzed photometry and gives, in parentheses, the number of $I$-magnitude measurements included in the analysis, and the rms error of the fit, $\sigma$, in units of mmag. Column (3) contains the derived mean magnitudes, columns (4)-(6) give the dominant and additional pulsation periods and their ratio, and columns (7)-(9) contain the Fourier first-term amplitudes and their ratios. The $A_{1, D}$ amplitudes for the six stars with the lowest amplitudes have been italicized. The bottom four stars were classified by the OGLE survey as 'RRc'; otherwise the classification was 'RRab'.

\begin{tabular}{|c|c|c|c|c|c|c|c|c|}
\hline $\begin{array}{l}\text { OGLE } \\
\text { no. } \\
(1)\end{array}$ & $\begin{array}{c}\text { Epochs }\left(\mathrm{N}_{I}, \sigma\right) \\
(2)\end{array}$ & $\begin{array}{c}\langle I> \\
{[\mathrm{mag}]} \\
(3)\end{array}$ & $\begin{array}{l}P_{D} \\
{[\mathrm{~d}]} \\
(4)\end{array}$ & $\begin{array}{l}P_{A} \\
{[\mathrm{~d}]} \\
(5)\end{array}$ & $\begin{array}{c}P_{A} / P_{D} \\
(6)\end{array}$ & $\begin{array}{c}A_{1, D} \\
{[\mathrm{mmag}]} \\
(7)\end{array}$ & $\begin{array}{c}A_{1, A} \\
{[\mathrm{mmag}]} \\
(8)\end{array}$ & $\begin{array}{c}A_{1, D} / A_{1, A} \\
\text { (9) }\end{array}$ \\
\hline 02943 & $2010-17(1665,35)$ & $17.460 \pm 0.001$ & $0.3367005(1)$ & $0.2297799(7)$ & $0.682446(2)$ & $189.0 \pm 1.2$ & $6.9 \pm 1.3$ & $27.5 \pm 5.3$ \\
\hline 15842 & $2001-17(1021,8)$ & $15.004 \pm 0.001$ & $0.36543510(2)$ & $0.2534121(2)$ & $0.693453(1)$ & $171.4 \pm 0.4$ & $7.9 \pm 0.4$ & $21.7 \pm 1.0$ \\
\hline 01401 & $2010-16(601,16)$ & $16.907 \pm 0.001$ & $0.3130437(1)$ & $0.2139031(4)$ & $0.683301(1)$ & $219.7 \pm 1.0$ & $13.4 \pm 1.0$ & $16.4 \pm 1.3$ \\
\hline 05851 & $2001-17(7330,52)$ & $18.124 \pm 0.001$ & $0.35468745(5)$ & $0.2456307(2)$ & $0.692527(1)$ & $195.6 \pm 0.9$ & $17.1 \pm 0.8$ & $11.4 \pm 0.6$ \\
\hline \multirow[t]{2}{*}{14135} & $2010-17(1847,38)$ & $17.583 \pm 0.001$ & $0.3008045(1)$ & $0.2153329(4)$ & $0.715857(2)$ & $176.3 \pm 1.3$ & $15.5 \pm 1.3$ & $11.4 \pm 0.9$ \\
\hline & & & $0.3008045(1)$ & $0.2244637(5)$ & $0.746211(2)$ & $176.3 \pm 1.3$ & $26.8 \pm 1.3$ & $6.58 \pm 0.93$ \\
\hline $12880 *$ & $2010-17(6586,29)$ & $16.692 \pm 0.001$ & $0.34004076(4)$ & $0.2390558(2)$ & $0.703021(1)$ & $210.1 \pm 0.5$ & $27.1 \pm 0.5$ & $7.74 \pm 0.17$ \\
\hline 08621 & $2010-13(457,22)$ & $16.255 \pm 0.001$ & $0.3300141(2)$ & $0.2294011(7)$ & $0.695125(3)$ & $195.4 \pm 1.6$ & $25.3 \pm 1.7$ & $7.71 \pm 0.57$ \\
\hline 30418 & $2010-16(556,33)$ & $18.002 \pm 0.002$ & $0.3640956(3)$ & $0.259372(1)$ & $0.712373(2)$ & $185.3 \pm 2.1$ & $27.5 \pm 2.1$ & $6.75 \pm 0.59$ \\
\hline 14063 & $2010-17(2013,13)$ & $16.245 \pm 0.001$ & $0.3753218(1)$ & $0.2634306(2)$ & $0.701879(1)$ & $155.9 \pm 0.4$ & $23.9 \pm 0.4$ & $6.52 \pm 0.13$ \\
\hline 30470 & $2010-14(159,22)$ & $17.262 \pm 0.002$ & $0.3811721(7)$ & $0.263756(2)$ & $0.691960(6)$ & $193.3 \pm 2.7$ & $29.9 \pm 3.3$ & $6.46 \pm 0.79$ \\
\hline 28160 & $2010-17(927,38)$ & $17.056 \pm 0.001$ & $0.3268868(2)$ & $0.2329577(4)$ & $0.712656(2)$ & $160.8 \pm 1.8$ & $25.2 \pm 1.8$ & $6.38 \pm 0.54$ \\
\hline 24500 & $2010-17(776,46)$ & $18.340 \pm 0.002$ & $0.3385730(3)$ & $0.2404183(6)$ & $0.710093(2)$ & $165.9 \pm 2.4$ & $26.5 \pm 2.4$ & $6.27 \pm 0.67$ \\
\hline 29286 & $2010-17(681,42)$ & $18.142 \pm 0.002$ & $0.3882796(3)$ & $0.2707951(5)$ & $0.697423(2)$ & $180.2 \pm 2.4$ & $31.1 \pm 2.4$ & $5.79 \pm 0.53$ \\
\hline 19058 & $2010-17(568,29)$ & $17.724 \pm 0.001$ & $0.3060771(1)$ & $0.2140382(3)$ & $0.699295(1)$ & $178.7 \pm 1.8$ & $31.8 \pm 1.9$ & $5.61 \pm 0.40$ \\
\hline $32721 *$ & $2010-16(287,31)$ & $17.060 \pm 0.002$ & $0.3261655(3)$ & $0.2301606(5)$ & $0.705656(2)$ & $178.4 \pm 3.2$ & $32.6 \pm 3.2$ & $5.47 \pm 0.63$ \\
\hline 02990 & $2001-17(1003,45)$ & $17.832 \pm 0.001$ & $0.3530475(1)$ & $0.2476270(3)$ & $0.701399(1)$ & $161.1 \pm 2.1$ & $29.7 \pm 2.0$ & $5.42 \pm 0.48$ \\
\hline 02217 & $2010-17(833,18)$ & $16.392 \pm 0.001$ & $0.3655628(2)$ & $0.2569971(4)$ & $0.703018(1)$ & $115.8 \pm 0.9$ & $22.4 \pm 0.9$ & $5.18 \pm 0.26$ \\
\hline 30974 & $2010-16(556,33)$ & $17.660 \pm 0.002$ & $0.2897323(1)$ & $0.2042718(3)$ & $0.705036(1)$ & $208.0 \pm 2.3$ & $40.2 \pm 2.3$ & $5.17 \pm 0.33$ \\
\hline 02147 & $2010-17(834,17)$ & $16.723 \pm 0.001$ & $0.3522055(1)$ & $0.2506667(2)$ & $0.711706(1)$ & $178.5 \pm 0.9$ & $35.2 \pm 0.9$ & $5.08 \pm 0.15$ \\
\hline $03090 *$ & $2010-17(1758,52)$ & $17.221 \pm 0.001$ & $0.3243208(2)$ & $0.2308657(3)$ & $0.711844(1)$ & $171.1 \pm 1.7$ & $33.7 \pm 1.7$ & $5.07 \pm 0.28$ \\
\hline 24360 & $2010-17(648,37)$ & $17.933 \pm 0.002$ & $0.3265030(2)$ & $0.2300291(4)$ & $0.704524(2)$ & $179.1 \pm 2.3$ & $36.4 \pm 2.1$ & $4.92 \pm 0.38$ \\
\hline $01529 *$ & $2001-17(1048,29)$ & $16.934 \pm 0.001$ & $0.3633225(1)$ & $0.2545659(2)$ & $0.700661(1)$ & $173.5 \pm 1.3$ & $35.3 \pm 1.3$ & $4.91 \pm 0.22$ \\
\hline $19121 *$ & $2010-17(815,35)$ & $17.462 \pm 0.002$ & $0.3575967(2)$ & $0.2481799(4)$ & $0.694022(1)$ & $158.4 \pm 1.8$ & $33.1 \pm 1.8$ & $4.79 \pm 0.31$ \\
\hline $09117 *$ & $2010-17(14998,23)$ & $16.626 \pm 0.001$ & $0.30967722(2)$ & $0.2190973(1)$ & $0.707502(1)$ & $185.4 \pm 0.3$ & $39.4 \pm 0.3$ & $4.71 \pm 0.04$ \\
\hline 20645 & $2011-17(426,32)$ & $17.752 \pm 0.002$ & $0.3564776(2)$ & $0.2462885(5)$ & $0.690895(2)$ & $171.8 \pm 3.1$ & $37.2 \pm 2.7$ & $4.62 \pm 0.38$ \\
\hline $13745 *$ & $2010-17(1868,32)$ & $16.386 \pm 0.001$ & $0.3567739(1)$ & $0.2476187(2)$ & $0.694049(1)$ & $175.7 \pm 1.1$ & $39.3 \pm 1.0$ & $4.47 \pm 0.15$ \\
\hline $22305 *$ & $2010-17(780,67)$ & $18.514 \pm 0.003$ & $0.3406534(3)$ & $0.2381711(5)$ & $0.699160(2)$ & $202.6 \pm 3.6$ & $46.9 \pm 3.7$ & $4.32 \pm 0.42$ \\
\hline $16999 *$ & $2010-17(223,33)$ & $16.226 \pm 0.003$ & $0.3258227(3)$ & $0.2314176(6)$ & $0.710256(2)$ & $175.6 \pm 3.8$ & $40.8 \pm 3.6$ & $4.31 \pm 0.47$ \\
\hline$\overline{35838}$ & $2010-16(406,13)$ & $16.082 \pm 0.001$ & $0.31626814(7)$ & $0.2185164(2)$ & $0.690921(1)$ & $206.4 \pm 1.2$ & $48.6 \pm 1.0$ & $4.25 \pm 0.11$ \\
\hline 07688 & $2010-17(1549,27)$ & $17.610 \pm 0.001$ & $0.31543654(8)$ & $0.2181543(2)$ & $0.691595(1)$ & $195.1 \pm 1.0$ & $47.2 \pm 1.0$ & $4.13 \pm 0.11$ \\
\hline 14481 & $2010-16(542,12)$ & $16.285 \pm 0.001$ & $0.4097129(1)$ & $0.2820237(3)$ & $0.688345(1)$ & $173.7 \pm 0.8$ & $45.4 \pm 0.8$ & $3.82 \pm 0.08$ \\
\hline $27203 *$ & $2010-16(404,36)$ & $17.701 \pm 0.002$ & $0.3211947(2)$ & $0.2233162(4)$ & $0.695267(2)$ & $162.5 \pm 3.1$ & $43.6 \pm 2.8$ & $3.72 \pm 0.31$ \\
\hline 32923 & $2010-17(526,46)$ & $18.270 \pm 0.002$ & $0.3492864(3)$ & $0.2450249(4)$ & $0.701501(2)$ & $162.8 \pm 3.1$ & $44.9 \pm 3.1$ & $3.63 \pm 0.32$ \\
\hline$\underline{00595}$ & $2010-17(824,12)$ & $16.771 \pm 0.001$ & $0.2818316(1)$ & $0.1997130(1)$ & $0.708625(1)$ & $112.5 \pm 0.7$ & $31.7 \pm 0.6$ & $3.55 \pm 0.09$ \\
\hline 21400 & $2010-17(893,32)$ & $17.940 \pm 0.001$ & $0.3527255(2)$ & $0.2538858(3)$ & $0.719783(1)$ & $152.6 \pm 1.6$ & $43.5 \pm 1.6$ & $3.50 \pm 0.16$ \\
\hline 15657 & $2010-16(156,28)$ & $16.010 \pm 0.007$ & $0.3665869(5)$ & $0.2540171(7)$ & $0.692925(3)$ & $180.0 \pm 3.6$ & $55.1 \pm 5.0$ & $2.81 \pm 0.22$ \\
\hline 00617 & $2001-17(933,52)$ & $18.442 \pm 0.003$ & $0.3381064(1)$ & $0.2417129(2)$ & $0.714902(1)$ & $168.8 \pm 2.6$ & $61.2 \pm 2.6$ & $2.76 \pm 0.16$ \\
\hline 18798 & $2010-15(114,35)$ & $18.174 \pm 0.013$ & $0.3686818(9)$ & $0.260384(1)$ & $0.706255(5)$ & $181 \pm 12$ & $54 \pm 13$ & $2.5 \pm 0.6$ \\
\hline 30908 & $2010-17(5849,88)$ & $18.635 \pm 0.001$ & $0.2874647(2)$ & $0.2031463(2)$ & $0.706683(1)$ & $99.5 \pm 1.6$ & $38.0 \pm 1.6$ & $2.62 \pm 0.16$ \\
\hline 19847 & $2010-14(111,31)$ & $18.264 \pm 0.005$ & $0.3177105(9)$ & $0.224449(1)$ & $0.706457(6)$ & $136.1 \pm 6.6$ & $59.0 \pm 6.5$ & $2.31 \pm 0.37$ \\
\hline 34006 & $2010-17(1852,34)$ & $17.988 \pm 0.001$ & $0.3120725(2)$ & $0.2194600(2)$ & $0.703234(1)$ & $90.4 \pm 1.2$ & $43.7 \pm 1.2$ & $2.07 \pm 0.08$ \\
\hline 31754 & $2010-17(6707,41)$ & $18.022 \pm 0.001$ & $0.3173163(1)$ & $0.2259796(1)$ & $0.712159(1)$ & $88.9 \pm 0.7$ & $77.9 \pm 0.7$ & $1.14 \pm 0.02$ \\
\hline
\end{tabular}

To sum up, the linear models of Chadid et al. and Smolec et al. fail to describe the pRRd stars in two ways: (1) they do not reproduce period ratios below 0.71 ; and (2) the pulsation modes are not unstable for the short periods of the pRRd stars, i.e., the models lie outside of the instability strip. The period ratio discrepancy can be easily fixed by assuming $[\mathrm{Fe} / \mathrm{H}]>0.0$ dex, but increasing $[\mathrm{Fe} / \mathrm{H}]$ most likely will not fix the growth-rates discrepancy at the short periods. In fact, it could make it worse - judging from fig. 10 of the P17 paper the shortest period at which both radial modes are unstable becomes even longer as the $[\mathrm{Fe} / \mathrm{H}]$ increases.

The Chadid et al. (2010) models were computed for their analysis of the 15th-magnitude star V1127 Aql. With dominant period $P_{0}=0.3560 \mathrm{~d}$, additional period $P_{1}=0.2480 \mathrm{~d}$, and period ratio $P_{1} / P_{0}=0.6966$, its location in the Petersen diagram is in the zone occupied by pRRd stars (see Fig. 1 where it is represented by a blue cross).
Based on the observed periods and the calculated models V1127 Aql was considered by Chadid et al. to be a possible double-mode RR Lyrae star, but was rejected as such on the grounds that the period ratio does not fit to the relation defined by the cRRd stars of our Galaxy. Finally, V1127 Aql also lies in the direction of the Galactic Bulge $(l=38.1804$, $b=-6.458)$, and like many of the known pRRd stars it too exhibits a Blazhko modulation $\left(P_{\mathrm{BL}}=26.9 \mathrm{~d}\right)$. Thus, the amplitudes for both modes are variable, as is the case for some of the pRRd stars, e.g., OGLE-BLG-RRLYR-09117. We conclude that V1127 Aql almost certainly is another member of the group of 'peculiar' RRd stars.

Another possible pRRd star is AH Cam. With dominant period $P_{\mathrm{L}}=0.368716 \mathrm{~d}$, secondary period $P_{\mathrm{S}}=0.2628 \mathrm{~d}$, and $P_{\mathrm{S}} / P_{\mathrm{L}}=0.7130$, it too lies in the $\mathrm{pRRd}$ region of the Petersen diagram (see Fig. 1). It is metal-rich with $[\mathrm{Fe} / \mathrm{H}]$ $>-0.5$ dex and like many pRRd stars it exhibits Blazhko variations $\left(P_{B}=10.8289 \pm 0.0002 \mathrm{~d}\right.$ - see Smith et al. 1994; 

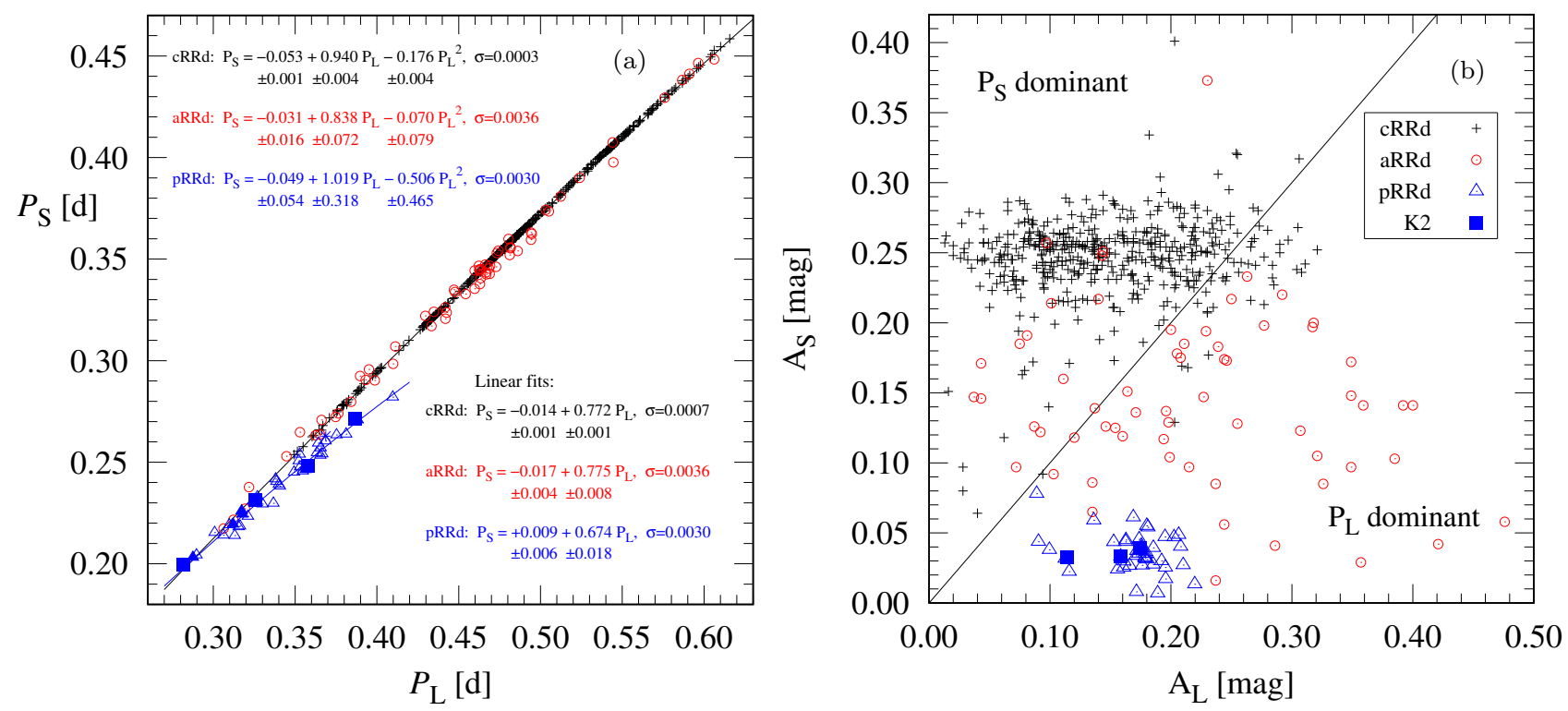

Figure 15. (a) $P_{L}-P_{S}$ graph comparing the longer and shorter pulsation periods for the RRd stars in Figs. 1-2. The four pRRd stars observed by $K_{2}$ are plotted with large blue squares, and the blue and black 'lines' through the pRRd and cRRd points are fitted leastsquares quadratic curves, the equations of which (along with linear fit equations) are given on the graph. (b) $A_{L}-A_{S}$ graph comparing the Fourier first-term $I$-amplitudes of the longer- and shorter-period modes for the RRd stars in Figs. 1-2. The diagonal 1:1 line separates the $P_{S}$-dominant from the $P_{L}$-dominant RRd stars. EPIC 216764000 is absent because no OGLE $I$-passband photometry exists for it.

Le Borgne et al. 2012). The amplitude of the dominant mode, $A_{1, D}=201.3 \mathrm{mmag}$, too, is similar to that for $\mathrm{pRRd}$ stars.

Also of interest are the three short-period aRRd stars (red circles) that reside in Fig. 1 among the pRRd stars. The three stars were discovered and classified by Soszyński et al. (2019) and have OGLE identifications OGLE-BLG-RRLYR-60063, OGLE-GD-RRLYR-02968 and OGLE-GD-RRLYR-08752. Unlike the pRRd stars for which the additional amplitudes are always much smaller than the dominant amplitudes (see $\S 4.2$ ) the primary and secondary amplitudes for the three stars are of comparable value and relatively large (see fig. 4 of Soszyński et al. 2019). In this sense all three are more like very-short-period cRRd stars than pRRd stars, which would make them the shortestperiod cRRd stars yet identified and thus of considerable interest. With mean $I$ magnitudes ranging from 14.6 to 16.2 their metallicities might be measurable using spectra taken with a large telescope.

In Figure 15(a) the Petersen diagram (Fig. 1) is viewed from another perspective. In the $P_{L}-P_{S}$ diagram the two periods for all three types of $\mathrm{RRd}$ stars appear to be linearly correlated, the equations of which are given on the graph. Inspection of the residuals revealed that for the cRRd stars the correlation is non-linear, a result confirmed when the cRRd points were fit with a quadratic formula, the rms-error derived from the quadratic fit being less than half the size of the rms-error derived from the linear fit, i.e., $\sigma=0.0003$ vs. 0.0007. When quadratics were fit to the pRRd and aRRd points their $\sigma$ values remained unchanged, suggesting that linear fits are sufficient for describing their $P_{L}-P_{S}$ relations. The aRRd relation resembles the cRRd relation but with larger scatter. Given the large cloud-like nature of the aRRd distribution in the Petersen diagram this result was some- what surprising. Fig. 15(a) also shows that for the pRRd stars the slope of the $P_{L}-P_{S}$ relation, $0.674 \pm 0.018$, is significantly shallower than that for the cRRd (and aRRd) stars, $0.772 \pm 0.001$. If the pRRd stars are simply shortperiod cRRd stars this would suggest that for a given $P_{L}$ the 'additional' period of the pRRd stars is shorter than expected. We regard the different slopes as strong evidence that there is a fundamental difference between the pRRd and the cRRd (or aRRd) stars.

In the Petersen diagram (Fig. 1) the mean curve (black) plotted through the cRRd points follows from the Fig. 15(a) fitted quadratic $P_{L}-P_{S}$ relation. Extrapolation of the trendline to periods shorter than $0.35 \mathrm{~d}$ shows that almost all of the pRRd stars lie below the extrapolated cRRd curve. The corresponding mean trendline through the pRRd data points was not plotted in Fig. 1 (owing to the highly uncertain first and third coefficients for the pRRd quadratic fit).

\subsection{Light Curve Amplitudes and Shapes}

The original 42 pRRd stars were found by P17 to divide into two groups based on the Fourier first-term amplitudes of the dominant oscillations: 36 stars with amplitudes in the range $140<A_{1, D}<220 \mathrm{mmag}$, and six stars with amplitudes $<140$ mmag (see fig. 2 of P17). Our analysis of the larger 2001-2017 OGLE data set confirms this result, in particular the low $A_{1, D}$ amplitudes for the six low-amplitude stars (see column 7 of Table 8). P17 also found that when total amplitudes and Fourier amplitude parameters $R_{21}$ and $R_{31}$ for the dominant oscillations are plotted vs. $P_{L}$ the division into two groups persists (see figs. 2 and 3 of P17), although amplitude ratios are low only in some of the low-amplitude pRRd stars. However, the apparent division went away in the amplitude-independent $P_{L-} \phi_{21}^{S}$ and $P_{L-} \phi_{31}^{S}$ phase-difference 
Table 9. Fourier decomposition amplitude-ratio, $R_{21}$, and phase-difference, $\phi_{21}^{c}$, parameters for the long-period $\left(P_{L}\right)$ and short-period $\left(P_{S}\right)$ pulsation modes of the $42 \mathrm{pRRd}$ stars with OGLE-survey $I$-photometry. Also given are the derived light-curve-shape indices, $\Delta R_{21}$ and $\Delta \phi_{21}$ (see text for definitions, and Fig.16). As in Table 8 the star numbers are underlined for the three stars observed by K2, and followed by an asterisk if either mode exhibits Blazhko modulations.

\begin{tabular}{|c|c|c|c|c|c|c|c|c|}
\hline $\begin{array}{c}\text { OGLE } \\
(1)\end{array}$ & $\begin{array}{l}P_{L}[\mathrm{~d}] \\
\quad(2)\end{array}$ & $\begin{array}{c}P_{S} / P_{L} \\
(3)\end{array}$ & $\begin{array}{l}R_{21, \mathrm{~L}} \\
\quad(4)\end{array}$ & $\begin{array}{c}R_{21, \mathrm{~S}} \\
(5)\end{array}$ & $\begin{array}{c}\Delta R_{21} \\
(6)\end{array}$ & $\begin{array}{c}\phi_{21, L}^{c}, \\
(7)\end{array}$ & $\begin{array}{c}\phi_{21, S}^{c}, \\
(8)\end{array}$ & $\begin{array}{c}\Delta \phi_{21} \\
(9)\end{array}$ \\
\hline 595 & 0.2818316 & 0.708625 & $0.387 \pm 0.006$ & $0.134 \pm 0.021$ & $0.25 \pm 0.02$ & $4.477 \pm 0.018$ & $4.71 \pm 0.16$ & $-0.23 \pm 0.16$ \\
\hline$\overline{617}$ & 0.3381064 & 0.714902 & $0.320 \pm 0.016$ & $0.083 \pm 0.042$ & $0.24 \pm 0.04$ & $4.530 \pm 0.056$ & $4.29 \pm 0.50$ & $0.24 \pm 0.50$ \\
\hline 1401 & 0.3130437 & 0.683301 & $0.386 \pm 0.005$ & $0.193 \pm 0.077$ & $0.19 \pm 0.08$ & $4.485 \pm 0.015$ & $3.44 \pm 0.42$ & $1.05 \pm 0.42$ \\
\hline $1529 *$ & 0.3633225 & 0.700661 & $0.352 \pm 0.008$ & $0.064 \pm 0.037$ & $0.29 \pm 0.04$ & $4.592 \pm 0.025$ & $5.25 \pm 0.58$ & $-0.66 \pm 0.58$ \\
\hline 2147 & 0.3522055 & 0.711706 & $0.324 \pm 0.005$ & $0.093 \pm 0.026$ & $0.23 \pm 0.03$ & $4.620 \pm 0.018$ & $4.35 \pm 0.29$ & $0.27 \pm 0.29$ \\
\hline 2217 & 0.3655628 & 0.703018 & $0.319 \pm 0.009$ & $0.135 \pm 0.043$ & $0.18 \pm 0.04$ & $4.584 \pm 0.029$ & $4.37 \pm 0.31$ & $0.22 \pm 0.31$ \\
\hline 2943 & 0.3367005 & 0.682446 & $0.340 \pm 0.007$ & $0.255 \pm 0.190$ & $0.09 \pm 0.19$ & $4.559 \pm 0.023$ & $2.91 \pm 0.79$ & $1.65 \pm 0.79$ \\
\hline 2990 & 0.3530475 & 0.701399 & $0.291 \pm 0.014$ & $0.123 \pm 0.070$ & $0.17 \pm 0.07$ & $4.588 \pm 0.051$ & $3.96 \pm 0.58$ & $0.62 \pm 0.58$ \\
\hline $3090 *$ & 0.3243208 & 0.711844 & $0.317 \pm 0.010$ & $0.036 \pm 0.050$ & $0.28 \pm 0.05$ & $4.580 \pm 0.037$ & $5.41 \pm 1.37$ & $-0.83 \pm 1.38$ \\
\hline 5851 & 0.3546875 & 0.692527 & $0.364 \pm 0.005$ & $0.086 \pm 0.050$ & $0.28 \pm 0.05$ & $4.562 \pm 0.015$ & $5.15 \pm 0.59$ & $-0.59 \pm 0.59$ \\
\hline 7688 & 0.3154365 & 0.691595 & $0.359 \pm 0.005$ & $0.026 \pm 0.021$ & $0.33 \pm 0.02$ & $4.521 \pm 0.017$ & $3.85 \pm 0.82$ & $0.67 \pm 0.82$ \\
\hline 8621 & 0.3300003 & 0.695144 & $0.314 \pm 0.023$ & $0.246 \pm 0.144$ & $0.07 \pm 0.15$ & $4.537 \pm 0.080$ & $6.56 \pm 0.61$ & $-2.02 \pm 0.61$ \\
\hline $9117 *$ & 0.3096772 & 0.707502 & $0.379 \pm 0.002$ & $0.102 \pm 0.007$ & $0.28 \pm 0.01$ & $4.468 \pm 0.005$ & $5.04 \pm 0.07$ & $-0.57 \pm 0.07$ \\
\hline $12880 *$ & 0.3400408 & 0.703021 & $0.380 \pm 0.003$ & $0.168 \pm 0.019$ & $0.21 \pm 0.02$ & $4.534 \pm 0.008$ & $3.93 \pm 0.12$ & $0.60 \pm 0.12$ \\
\hline $13745 *$ & 0.3567739 & 0.694049 & $0.340 \pm 0.006$ & $0.044 \pm 0.027$ & $0.30 \pm 0.03$ & $4.620 \pm 0.022$ & $6.19 \pm 0.62$ & $-1.57 \pm 0.62$ \\
\hline 14063 & 0.3753218 & 0.701879 & $0.307 \pm 0.003$ & $0.125 \pm 0.017$ & $0.18 \pm 0.02$ & $4.679 \pm 0.010$ & $4.31 \pm 0.14$ & $0.37 \pm 0.14$ \\
\hline 14135 & 0.3008045 & 0.715857 & $0.339 \pm 0.008$ & $0.066 \pm 0.083$ & $0.27 \pm 0.08$ & $4.599 \pm 0.026$ & $5.31 \pm 1.27$ & $-0.71 \pm 1.27$ \\
\hline 14481 & 0.4097129 & 0.688345 & $0.333 \pm 0.005$ & $0.075 \pm 0.017$ & $0.26 \pm 0.02$ & $4.680 \pm 0.016$ & $4.09 \pm 0.23$ & $0.59 \pm 0.23$ \\
\hline 15657 & 0.3665869 & 0.692925 & $0.407 \pm 0.044$ & $0.175 \pm 0.091$ & $0.23 \pm 0.10$ & $4.81 \pm 0.10$ & & \\
\hline 15842 & 0.3654351 & 0.693453 & $0.320 \pm 0.002$ & $0.052 \pm 0.046$ & $0.27 \pm 0.05$ & $4.570 \pm 0.008$ & $5.02 \pm 0.87$ & $-0.45 \pm 0.87$ \\
\hline $16999 *$ & 0.3258227 & 0.710256 & $0.388 \pm 0.024$ & $0.125 \pm 0.092$ & $0.26 \pm 0.10$ & $4.566 \pm 0.068$ & $3.90 \pm 0.68$ & $0.67 \pm 0.69$ \\
\hline 18798 & 0.3686818 & 0.706255 & $0.483 \pm 0.140$ & & & $4.82 \pm 0.30$ & & \\
\hline 19058 & 0.3060771 & 0.699295 & $0.362 \pm 0.011$ & $0.039 \pm 0.057$ & $0.32 \pm 0.06$ & $4.598 \pm 0.068$ & $5.41 \pm 1.57$ & $-0.81 \pm 1.58$ \\
\hline $19121 *$ & 0.3575967 & 0.694022 & $0.342 \pm 0.012$ & $0.042 \pm 0.054$ & $0.30 \pm 0.07$ & $4.577 \pm 0.040$ & $3.51 \pm 1.28$ & $1.06 \pm 1.28$ \\
\hline 19847 & 0.3177105 & 0.706457 & $0.275 \pm 0.052$ & $0.281 \pm 0.128$ & $-0.01 \pm 0.14$ & $4.36 \pm 0.18$ & $4.51 \pm 0.33$ & $-0.15 \pm 0.38$ \\
\hline 20645 & 0.3564776 & 0.690895 & $0.367 \pm 0.018$ & $0.161 \pm 0.071$ & $0.21 \pm 0.07$ & $4.675 \pm 0.056$ & $4.21 \pm 0.50$ & $0.47 \pm 0.50$ \\
\hline 21400 & 0.3527255 & 0.719783 & $0.357 \pm 0.011$ & $0.051 \pm 0.037$ & $0.31 \pm 0.04$ & $4.556 \pm 0.035$ & $4.01 \pm 0.73$ & $0.54 \pm 0.73$ \\
\hline $22305 *$ & 0.3406534 & 0.699160 & $0.315 \pm 0.019$ & $0.049 \pm 0.078$ & $0.27 \pm 0.08$ & $4.598 \pm 0.068$ & & \\
\hline 24360 & 0.3265030 & 0.704524 & $0.337 \pm 0.013$ & $0.059 \pm 0.059$ & $0.28 \pm 0.06$ & $4.506 \pm 0.044$ & $4.13 \pm 1.08$ & $0.37 \pm 1.09$ \\
\hline 24500 & 0.3385730 & 0.710093 & $0.385 \pm 0.016$ & $0.220 \pm 0.097$ & $0.17 \pm 0.10$ & $4.546 \pm 0.048$ & $2.99 \pm 0.45$ & $1.55 \pm 0.45$ \\
\hline $27203 *$ & 0.3211947 & 0.695267 & $0.328 \pm 0.019$ & $0.143 \pm 0.068$ & $0.19 \pm 0.07$ & $4.582 \pm 0.074$ & $4.90 \pm 0.47$ & $-0.32 \pm 0.48$ \\
\hline 28160 & 0.3268868 & 0.712656 & $0.323 \pm 0.012$ & $0.129 \pm 0.075$ & $0.19 \pm 0.08$ & $4.617 \pm 0.043$ & $4.49 \pm 0.58$ & $0.13 \pm 0.59$ \\
\hline 29286 & 0.3882796 & 0.697423 & $0.302 \pm 0.014$ & $0.119 \pm 0.082$ & $0.18 \pm 0.08$ & $4.693 \pm 0.050$ & $3.63 \pm 0.64$ & $1.06 \pm 0.65$ \\
\hline 30418 & 0.3640956 & 0.712373 & $0.337 \pm 0.012$ & $0.166 \pm 0.079$ & $0.17 \pm 0.08$ & $4.534 \pm 0.042$ & $4.21 \pm 0.49$ & $0.32 \pm 0.50$ \\
\hline 30470 & 0.3811710 & 0.691950 & $0.266 \pm 0.036$ & $0.104 \pm 0.132$ & $0.16 \pm 0.14$ & $4.48 \pm 0.14$ & & \\
\hline 30908 & 0.2874647 & 0.706683 & $0.276 \pm 0.017$ & $0.236 \pm 0.044$ & $0.04 \pm 0.05$ & $4.296 \pm 0.068$ & $5.06 \pm 0.20$ & $-0.76 \pm 0.21$ \\
\hline 30974 & 0.2897323 & 0.705036 & $0.367 \pm 0.012$ & $0.039 \pm 0.053$ & $0.33 \pm 0.05$ & $4.466 \pm 0.035$ & $3.92 \pm 1.42$ & $0.54 \pm 1.42$ \\
\hline 31754 & 0.3173163 & 0.712159 & $0.181 \pm 0.008$ & $0.131 \pm 0.009$ & $0.05 \pm 0.01$ & $4.403 \pm 0.047$ & $5.02 \pm 0.07$ & $-0.61 \pm 0.09$ \\
\hline $32721 *$ & 0.3261655 & 0.705656 & $0.330 \pm 0.019$ & $0.076 \pm 0.101$ & $0.25 \pm 0.10$ & $4.406 \pm 0.068$ & & \\
\hline 32923 & 0.3492864 & 0.701501 & $0.320 \pm 0.020$ & $0.095 \pm 0.070$ & $0.23 \pm 0.07$ & $4.590 \pm 0.072$ & $5.00 \pm$ & $-0.41 \pm 0.73$ \\
\hline 34006 & 0.3120725 & 0.703234 & $0.221 \pm 0.013$ & $0.131 \pm 0.028$ & $0.09 \pm 0.03$ & $4.363 \pm 0.062$ & $5.11 \pm 0.22$ & $-0.74 \pm 0.23$ \\
\hline 35838 & 0.3162681 & 0.690921 & $0.374 \pm 0.006$ & $0.062 \pm 0.022$ & $0.31 \pm 0.02$ & $4.552 \pm 0.017$ & $3.75 \pm 0.36$ & $0.81 \pm 0.36$ \\
\hline
\end{tabular}

graphs shown in the bottom two panels of the same figure, where both indices form tight progressions with all the pRRd stars having similar $\phi_{21}^{s}$ and $\phi_{31}^{s}$ characteristics. In fact, in all the various fig. 3 graphs of P17 the locations of the higheramplitude pRRd stars lie between those of the single-mode RRc and RRab pulsators. Turning to the phased light curves for the dominant mode, after filtering out the contributions from the additional mode, all 42 stars were seen to have asymmetric dominant light curves (see fig. 4 of P17). Based on the entirety of the observations P17 concluded that despite their very short periods, $P_{L}=0.28-0.41 \mathrm{~d}$, the dominant oscillations of all $42 \mathrm{pRRd}$ stars correspond to fundamentalmode pulsation.

On the other hand the pulsation modes of the additional oscillations were left unexplained. Also uncertain were the shapes of the secondary light curves. Due in large part to the relatively low amplitudes (see fig. 2 of P17, and column 8 of our Table 8), and in the absence of high-precision photometry, P17 assumed sinusoidal light curve shapes for the additional pulsations. We can now say, based on our analysis of the high-precision Kepler photometry, that none of the four pRRd stars observed by K2 has a sinusoidal secondary light curve. Perhaps the strongest evidence of the asymmetries is given by the risetimes summarized in Table 4 . While a sinusoidal light curve would have $\mathrm{RT}=0.50$, the four $K 2$ stars have risetimes for the 'additional' oscillations, $\mathrm{RT}_{A}$, ranging from $0.421 \pm 0.002$ to $0.472 \pm 0.001$. By way of comparison the light curves of the dominant longer-period oscillations for the same four stars are much more asymmetric and have $\mathrm{RT}_{D}$ values ranging from $0.235 \pm 0.001$ to $0.289 \pm 0.001$. The non-sinusoidal nature of the secondary oscillations is also evident in the low but non-zero Fourier $R_{21}$ values, which for the four $K 2$ stars range from $0.048 \pm 0.005$ to $0.15 \pm 0.04$, and are significantly smaller than the values for the dominant oscillations, which range from $0.324 \pm 0.008$ to $0.398 \pm 0.001$ (see Table 4).

Not considered by P17 was how the amplitudes and amplitude ratios of the pRRd stars compare with those for cRRd (and aRRd) stars. The $P_{L}-A_{S} / A_{L}$ diagram plotted in Fig. 2 shows that the amplitude ratios of the cRRd stars tend to decrease as $P_{L}$ decreases, with a possible discontinuity occuring around $P_{L} \sim 0.45 \mathrm{~d}$, and that this trend appears to continue through to the pRRd stars. If the sample were limited to classical RRd stars in globular clusters one would see a clear trend in going from the long-period low-metallicity OoII cRRd stars to the intermediate-period intermediatemetallicity OoI cRRd stars (e.g., fig. 2 of Clement, Ferance \& Simon 1993).

Another perspective on the information contained in Fig. 2 is given by the $A_{L}-A_{S}$ diagram plotted in Figure 15(b). According to this graph the fundamental difference between the pRRd and cRRd stars is that the shorter-period addi- 

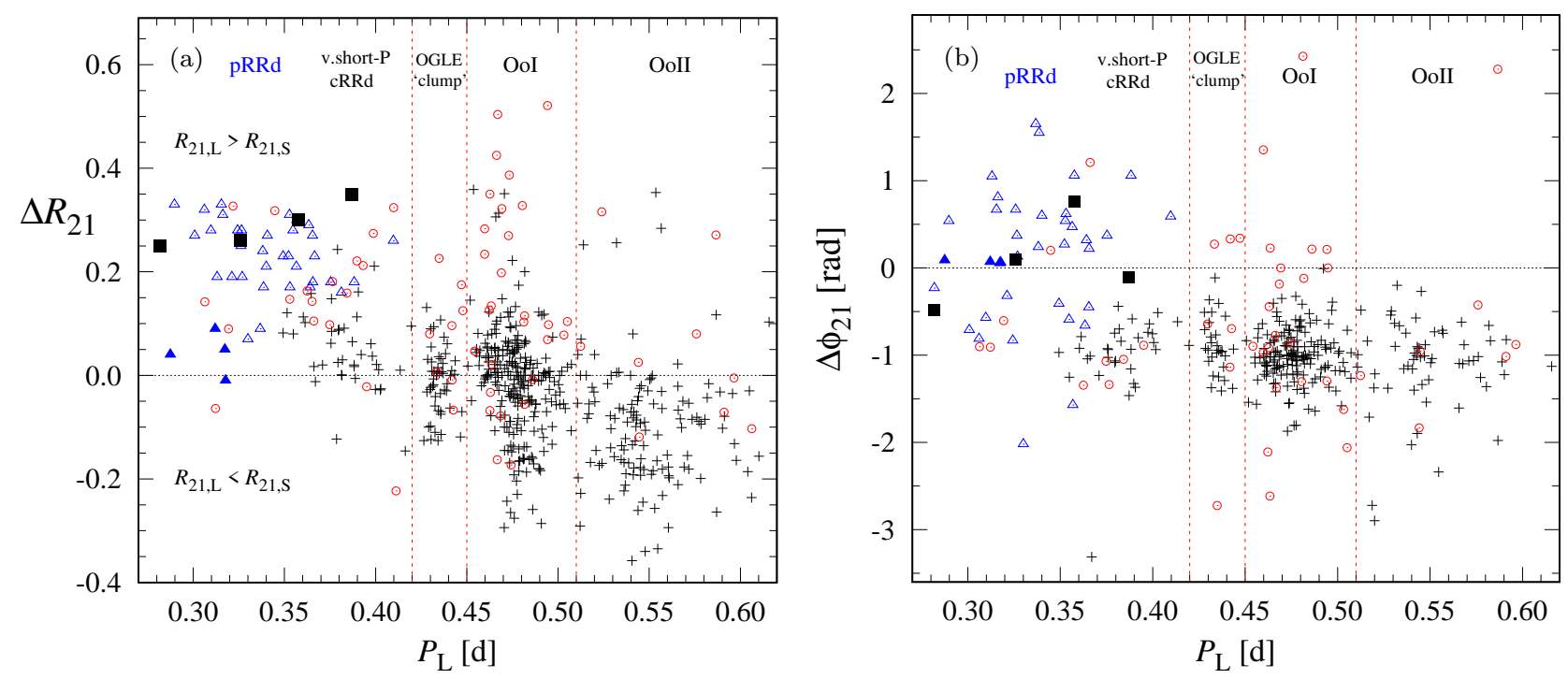

Figure 16. (a) $\Delta R_{21}$ vs. $P_{\mathrm{L}}$ diagram, and (b) $\Delta \phi_{21}$ vs. $P_{\mathrm{L}}$ diagram, for the pRRd stars (blue triangles, and large black-filled squares for the four stars observed by K2), cRRd stars (black plus signs) and aRRd stars (red open circles) in Figs. 1-2. The Fourier amplitude ratios and phase differences are all derived from OGLE $I$-photometry (except the points for EPIC 216764000 which were derived from the K2 photometry). Typical measurement rms-errors for the pRRd stars are \pm 0.06 for $\Delta R_{21}$ and \pm 0.66 rad for $\Delta \phi_{21}$; the 0GLE online catalog does not report measurement errors for cRRd and aRRd stars.

tional amplitudes, $A_{S}$, of all the pRRd stars are significantly lower than the $A_{S}$ amplitudes of almost all of the cRRd stars. Even the shortest-period cRRd stars which have periods and period ratios most similar to the pRRd stars, many of which have $P_{L}$ dominant like the pRRd stars (see Fig. 2), have significantly larger $A_{S}$ amplitudes $(\sim 0.24 \mathrm{mag})$ than the $A_{S}$ amplitudes of the pRRd stars ( $\sim 0.03 \mathrm{mag}$ ). Turning to the amplitudes of the longer-period (fundamental) modes, the $A_{L}$ values of the pRRd stars and of the cRRd stars are on average not too different, and this is so despite the range of the $A_{L}$ values being twice as narrow for the former group of stars. This result again supports the P17 conclusion that the dominant oscillations probably are fundamental-mode radial pulsations.

Fig. 15(b) also shows that the main cause of the wide range of amplitude ratios for the cRRd stars (see Fig. 2) is the large range of amplitudes for the longer-period oscillations, $A_{L}=0.01-0.32 \mathrm{mag}$, the range of amplitudes for the shorter-period first-overtone mode being fairly small for most of the cRRd stars $\left(A_{S}=0.2-0.3 \mathrm{mag}\right)$. Almost all of the cRRd stars with $A_{S}<0.2 \mathrm{mag}$ also tend to have low $A_{L}$ values, i.e., a dominant first-overtone.

Table 9 contains Fourier decomposition $R_{21}$ and $\phi_{21}^{c}$ parameters (including error estimates) for the dominant longer-period and additional shorter-period pulsation modes of the 42 pRRd stars, all derived from OGLE $I$-photometry; neither parameter was computed by P17 for either mode. Columns (6) and (9) contain two additional indices, both of which relate to the shapes of the light curves: $\Delta R_{21}$, defined as the difference between the $R_{21}$ amplitude ratio for the two modes, i.e., $R_{21, \mathrm{~L}}-R_{21, \mathrm{~S}}$; and $\Delta \phi_{21}$ defined as the difference between the $\phi_{21}^{c}$ phase difference for the two modes, i.e., $\phi_{21, \mathrm{~L}}^{c}-\phi_{21, \mathrm{~S}}^{c}$. The uncertainties given in Table 9 for the indices are only approximate (since they do not include possible covariance contributions).
The $P_{L}-\Delta R_{21}$ graph shown in Figure 16(a) compares the light curve shapes of the pRRd stars with those of cRRd and aRRd stars. The $\Delta R_{21}$ values for the cRRd and aRRd stars were computed from the $R_{21,1}$ and $R_{21,2}$ amplitude ratios reported in the OGLE online catalogue (note that since the OGLE catalog does not give error estimates for the reported Fourier parameters the uncertainties of the $\Delta R_{21}$ and $\Delta \phi_{21}$ values for the aRRd and cRRd stars in Fig.16 are unknown). The horizontal line at $\Delta R_{21}=0$ separates the RRd stars into those stars where $R_{21, \mathrm{~L}}<R_{21, \mathrm{~S}}$, which appears to be the case for almost all the OoII cRRd stars and half the OoI and OGLE-'clump' cRRd stars, and those with $R_{21, \mathrm{~L}}>R_{21, \mathrm{~S}}$, which is the case for the (metal-rich) veryshort-period cRRd stars, the aRRd stars, and the pRRd stars. Of particular interest here is the clear trend when moving from the longest- to the shortest-period RRd stars, which, if the pRRd and cRRd stars have distinctly different pulsation types, still requires a physical explanation.

Fourier phase-difference indices for the three types of RRd stars are compared in Figure 16(b). Almost all of the cRRd stars, regardless of the $P_{L}$ value, have negative differences, typically $\sim-1.0$. This is in striking contrast to two thirds of the pRRd stars and 11 of the aRRd stars that have positive $\Delta \phi_{21}$ values. Again this argues for the dissimilarity of the pRRd and very short-period cRRd stars.

\section{SUMMARY}

Four 'peculiar' RRd (pRRd) stars observed by the Kepler space telescope during NASA's K2 Mission have been identified. All four stars are located in the direction of the Galactic Bulge. Three of the stars are relatively faint (with $<V>=18-20 \mathrm{mag}$ ) and were found to be among the pRRd stars discovered by P17. The fourth star, EPIC 216764000 
(=V1125 Sgr), which is several magnitudes brighter than the other three stars, is a newly discovered pRRd star. Detailed frequency analyses of the high-precision long-cadence K2 photometry for all four stars were performed and the results used to study cycle-to-cycle light variations.

Both pulsation modes of EPIC 216764000 are observed to be non-stationary. Variations of the amplitudes and phases of the modes appear to be irregular and, at least within the length of the K2 data, are not repetitive. Besides the two modes, no additional frequencies are detected in the star, in particular, the 0.61 mode seen in all well-observed cRRd stars (Moskalik et al. 2018b; Nemec, Moskalik et al., in preparation) is not present. Owing to the relative brightness of EPIC 216764000 we urge that high-resolution spectra be taken to determine its chemical abundances, in particular, $[\mathrm{Fe} / \mathrm{H}]$.

For the three fainter pRRd stars our analysis of the K2 photometry and the OGLE photometry through 2017 has confirmed the dominant periods and Fourier parameters derived by Soszyński et al. (2017b). Also confirmed are the periods and amplitudes (both dominant and additional) derived by $\mathrm{P} 17$. The derived amplitudes and phases were used to calculate Fourier decomposition amplitude and phase parameters.

While P17 chose to compare the pRRd stars with singlemode RRc and RRab stars we have compared their periods, amplitudes and phases with those for classical and anomalous double-mode RR Lyrae stars. By viewing the Petersen diagram from the perspective of a $P_{L}-P_{S}$ graph we show that all three RRd star types obey roughly linear correlations between the two periods, and that the slope of the correlation for the pRRd stars is shallower than that for the cRRd and aRRd stars. In fact, the cRRd $P_{L}-P_{S}$ relation is better fit with a quadratic rather than a line. Various graphs are presented in support of the conclusion that the pRRd stars are not simply short-period cRRd stars. Finally, the galactic field stars V1127 Aql and AH Cam are identified as other probable members of the class of pRRd stars.

\section{ACKNOWLEDGEMENTS}

Funding for the Kepler/K2 Mission was provided by the NASA Science Mission directorate. We thank Emese Plachy and Laszlo Molnár for kindly supplying EAP-pipeline photometry for EPIC 216764000. We also thank Emese, Laszlo, Robert Szabó and Katrien Kolenberg for discussions of RRd stars observed during the Kepler/K2 Missions. JN acknowledges assistance from Dr. Amanda F. Linnell Nemec, and thanks the Camosun College Faculty Association for supporting his travel to various Kepler conferences. Interesting discussions were had with Radosław Poleski at the Kepler/K2 SciConIV meeting at the NASA-Ames Research Center in Mountain View, California. Finally we thank Prof. Johanna Jurcsik for her careful review of the paper and for her interesting suggestions for future work.

Data availability: the data underlying this article are available from the MAST website, at https://archive.stsci.edu/k2/, and all the datasets were derived from sources in the public domain.

\section{REFERENCES}

Alcock C. et al., 1997, ApJ, 482, 89

Alcock C. et al., 2000, ApJ, 542, 257

Benkő J.M. et al., 2010, MNRAS, 409, 1585

Benkő J.M., Plachy E., Szabó R., Molnár L., Kolláth Z., 2014, ApJS, 213, 31

Bernard E.J. et al., 2009, ApJ, 699, 1742

Borucki W. et al., 2010, Science, 327, 977

Breger M. et al., 1993, A\&A, 271, 482

Brown A.G.A. et al., 2018, A\&A, 616, A1

Butters O.W. et al., 2010, A\&A, 520, L10

Chadid M., 2012, A\&A, 540, A68

Chadid M. et al., 2010, A\&A, 510, A39

Chadid M. et al., 2014, AJ, 148, 88

Chadid M., Sneden Ch., Preston G.W., 2017, ApJ, 835, 187

Chambers K.C. et al., 2019, arXiv:1612.05560 4

Christy R., 1966, ApJ, 144, 108

Clement C.M., Nemec J.M., Robert N., Wells T., Dickens R.J., Bingham E.A., 1986, AJ, 92, 825

Clement C.M., Ferance S., Simon N.R., 1993, ApJ, 412, 183

Clement C.M., Hilditch R.W., Kałużny J. Ruciński S., 1997, ApJ, 489, L55

Clementini G., Corwin T.M., Carney B.W., Sumerel A.N., 2004, AJ, 127, 938

Clementini G. et al., 2019, A\&A, 622, A60

Cox A.N., King D.S., Hodson S.W., 1980, ApJ, 236, 219

Cox A.N., Hodson S.W., Clancy S.P., 1983, ApJ, 266, 94

Cseresnjes P., 2001, A\&A, 375, 909

Dziembowski W.A., 2012, Acta Astron., 62, 323

Dziembowski W.A., 2016, Comm. Konkoly Obs., 105, 23

Gavrilchenko T., Klein, Ch.R., Bloom J.S., Richards J.W., 2014, MNRAS, 441, 715

Gillet D., Crowe R.A. 1988, A\&A, 199, 242

Greer P.A., Payne S.G., Norton A.J., Maxted P.F.L., Smalley B., West R.G., Wheatley P.J., Kolb U.C., 2017, A\&A, 607, A11

Gruberbauer M. et al., 2007, MNRAS, 379, 1498

Handberg R., Lund, M.N., 2017, A\&A, 597, A36

Howell S.B. et al., 2014, PASP, 126, 398

Huber D. et al., 2016, ApJS, 224, 2

Jerzykiewicz M., Wenzel W., 1977, Acta Astron., 27, 35

Jurcsik J. et al., 2009, MNRAS, 400, 1006

Jurcsik J. et al., 2015, ApJS, 219, 25

Jurcsik J. et al., 2017, MNRAS, 468, 1317

Jurcsik J., Hajdu G., Dékány I., Nuspl J., Catelan M., Grebel E.K., 2018, MNRAS, 475, 4208

Kinemuchi K., Smith H.A., Woźniak P.R., McKay T.A., 2006, AJ, 132, 1202

Kolláth Z., 2018, Proc. Polish Astron. Soc., 6, 137

Kolláth Z., Buchler J.R., Szabó R., Csubry Z., 2002, A\&A, 385, 932

Kovács G., 2001a, A\&A, 375, 469

Kovács G., 2001b, ASSL, 257, 61

Kovács G., 2009, EAS Publ. Ser., 38, 91

Kovács G., 2018a, A\&A, 614, L4

Kovács G., 2018b, Proc. Polish Astron. Soc., 6, 129

Kurtz D.W., Bowman D.M., Ebo S.J., Moskalik P., Handberg R., Lund M.N., 2016, MNRAS, 455, 1237

Layden A.C., 1995, AJ, 110, 2288 
Le Borgne J.F. et al., 2007, A\&A, 476, 307

Le Borgne J.F. et al., 2012, AJ, 144, 39

Lenz P., Breger M., 2005, CoAst, 146, 53

Luger R., Agol E., Kruse E., Barnes R., Becker A., Foreman-Mackey D., Deming D., 2016, AJ, 152, 100

Lund M.N., Handberg R., Davies G.R., Chaplin W.J., Jones C.D., 2015, ApJ, 806, 30

Molnár L. et al., 2015, MNRAS, 452, 4283

Moskalik P., 2014, Proc. IAU Symp. 301, p. 249

Moskalik P., Kołaczkowski Z., 2009, MNRAS, 394, 1649

Moskalik P. et al., 2015, MNRAS, 447, 2348

Moskalik P., Nemec J.M., Molnár L., Plachy E., Szabó R., Kolenberg K., 2018a, Proc. Polish Astron. Soc., 6, 162

Moskalik P., Nemec J.M., Molnár L., Plachy E., Szabó R., Kolenberg K., 2018b, in Ballot J., Vauclairs S., Vauclair G., eds, Proc. PHOST Conf., available at https://zenodo.org/record/2204575\#.YBrs8-hKiUk

Mucciarelli A., 2014, Astron. Nachr., 335, 79

Nemec J.M., 1985a, AJ, 90, 204

Nemec J.M., 1985b, AJ, 90, 240

Nemec J.M., Clement C.M., 1989, AJ, 98, 860

Nemec J.M., Walker A., Jeon Y.-B., 2009, AJ, 138, 1310

Nemec J.M. et al., 2011, MNRAS, 417, 1022

Nemec J.M., Cohen J.G., Ripepi V., Derekas A., Moskalik P., Sesar B., Chadid M., Bruntt H., 2013, ApJ, 773, 181

Netzel H., Smolec R., 2019, MNRAS, 487, 5584

Netzel H., Smolec R., Moskalik P., 2015a, MNRAS, 447, 1173

Netzel H., Smolec R., Moskalik P., 2015b, MNRAS, 453, 2022

Netzel H., Smolec R., Soszyński I., Udalski A., 2018, MNRAS, 480, 1229

Petersen J.O., 1973, A\&A, 27, 89

Plachy E. et al., 2019, ApJS, 244, 32

Pojmański G., 1997, Acta Astron., 47, 467

Pojmański G., Maciejewski G., 2005, Acta Astron., 55, 97

Popielski B.L., Dziembowski W.A., Cassisi S., 2000, Acta Astron., 50, 491

Pollacco D.L. et al., 2006, PASP, 118, 1407

Preston G.W., 1959, ApJ, 130, 507

Prudil Z., Smolec R., Skarka M., Netzel H., 2017, MNRAS, 465, 4074 (P17)

Reegen P., 2007, A\&A, 467, 1353

Reegen P., 2011, CoAst, 163, 3

Sandage A., 2004, AJ, 128, 858

Sandage A., Katem B., Sandage M., 1981, ApJS, 46, 41

Simon N.R., Lee A.S., 1981, ApJ, 248, 291

Skrutskie M.F. et al., 2006, AJ, 131, 1163

Smith H.A., Matthews J.M., Lee K.M., Williams J., Silbermann N.A., Bolte M., 1994, AJ, 107, 679

Smith J.C. et al., 2012, PASP, 124, 1000

Smolec R. et al., 2015, MNRAS, 447, 3756

Smolec R., Prudil Z., Skarka M., Ba̧kowska K., 2016, MNRAS, 461, 2934

Smolec R., Moskalik P., Kałużny J., Pych W., Różyczka M., Thompson I.B., 2017a, MNRAS, 467, 2349

Smolec R., Dziembowski W., Moskalik P., Netzel H., Prudil Z., Skarka M., Soszyński I., 2017b, EPJ Web of Conf., 152, 06003
Sneden C. et al., 2018, AJ, 155, 45

Soszyński I. et al., 2009, Acta Astron., 59, 1

Soszyński I., Udalski A., Szymański M.K., Kubiak J.,

Pietrzyński G., Wyrzykowski Ł., Ulaczyk K., Poleski

R., 2010, Acta Astron., 60, 165

Soszyński I. et al., 2011, Acta Astron., 61, 1

Soszyński I. et al., 2014a, Acta Astron., 64, 1

Soszyński I. et al., 2014b, Acta Astron., 64, 177

Soszyński I. et al., 2016a, Acta Astron., 66, 131

Soszyński I. et al., 2016b, MNRAS, 463, 1332

Soszyński I. et al., 2017a, Acta Astron., 67, 103

Soszyński I. et al., 2017b, Acta Astron., 67, 297

Soszyński I. et al., 2019, Acta Astron., 69, 321

Stellingwerf R.F., 1978, ApJ, 224, 953

Stellingwerf R.F., 2011, Carnegie Obs. Ap. Ser., 5, 47

Still M., Barclay T., 2012, Astrophysics Source Code

Library, record ascl:1208.4

Szabó R., Kolláth Z., Buchler J.R., 2004, A\&A, 425, 627

Szabó R. et al., 2014, A\&A, 570, A100

Szczygieł D.M., Fabrycky D.C., 2007, MNRAS, 377, 1263

Szczygieł D.M., Pojmański G., Pilecki B., 2009, Acta Astron., 59, 137

Udalski A., Szymański M.K., Soszyński I., Poleski R., 2008, Acta Astron., 58, 69

Udalski A., Szymański M.K., Szymański G., 2015, Acta Astron., 65, 1

Uitterdijk J., 1949, Leiden Ann., 20, 41 